\title{
Type 2 Diabetes Mellitus Increases the Risk of Late- Onset Alzheimer's Disease: Obesity, Aging, Intersects and Continuum of Progression
}

\author{
Melvin R Hayden 1,2,* \\ 1 Diabetes and Cardiovascular Center, University of Missouri School of Medicine, Columbia, MO 65212, \\ USA \\ 2 Division of Endocrinology and Metabolism, Department of Medicine, University of Missouri, Columbia, \\ MO 65212, USA \\ * Correspondence: mrh29pete@gmail.com; 573-346-3019 USA
}

\begin{abstract}
Type 2 diabetes mellitus (T2DM) and late-onset Alzheimer's disease-dementia (LOAD) have become parallel global pandemics and current predictions indicate they will only increase over the coming decades. These pandemics may result from the coexistent increase of obesity and aging. T2DM is associated with cognitive impairments associated with both metabolic factors, diabetic cognopathy (DC) and an increased risk of LOAD. This review addresses possible mechanisms due to obesity, aging, intersects and mechanisms for the continuum of progression. Multiple ultrastructural images in female diabetic $d b / d b$ models are utilized to demonstrate marked cellular remodeling changes and provide for the discussion of functional changes in T2DM. Throughout this review multiple endeavors to demonstrate how T2DM increases the vulnerability of the brain's neurovascular unit (NVU), neuroglia and neurons are presented. It is difficult to condense so many links between T2DM and LOAD; however, five major intersections could be considered: $i$. aging (chronic age-related diseases); ii. metabolic (hyperglycemia - advanced glycation endproducts and its receptor (AGE/RAGE) interactions and hyperinsulinemia - insulin resistance (a linking linchpin); iii. oxidative stress (reactive oxygen-nitrogen species); $i v$. inflammation (peripheral macrophage and central brain microglia); $v$. vascular (macrovascular accelerated atherosclerosis - vascular stiffening and microvascular NVU remodeling with resulting impaired cerebral blood flow).
\end{abstract}

Keywords: aging; Alzheimer's disease; brain insulin resistance; $d b / d b$ diabetic mouse model; diabetic cognopathy; insulin resistance; metabolic syndrome; mixed dementia; obesity; type 2 diabetes mellitus

\section{Contents}

\section{OUTLINE}

\section{Abstract}

\section{Introduction}

1.1. T2DM, Late-onset Alzheimer's Disease-Dementia (LOAD) and Societal Aging

1.2. Aging, Obesity, Insulin Resistance, Metabolic Syndrome and the H and E Phenomenon

1.3. Hypothalamic-Pituitary-Adrenal (HPA) Axis Dysfunction in the Metabolic Syndrome: " $H$ " and " $E$ " Phenomenon

2. Multiple Intersects Between Type 2 Diabetes Mellitus and Late-Onset Alzheimer's Disease

3. Continuum of Progression: From Obesity, Insulin Resistance to T2DM to LOAD

3.1. Obesity

3.2. Capillary Neurovascular Unit (NVU

3.2.1. Endothelial Cell(s) (EC) - Endothelium

3.2.2. Endothelial Glycocalyx (ecGCx)

3.2.3. Basement Membrane (BM)

3.2.4. NVU Pericyte(s) (Pc) 


\subsubsection{Astrocyte (AC)}

\subsubsection{Microglial Cell (MGC)}

3.2.7. Oligodendrocyte and Myelin Remodeling in the diabetic female $\mathrm{db} / \mathrm{db}$

4. Oxidative Stress: Reactive Oxygen/Nitrogen Species (ROS/NOS) and Aberrant Mitochondria

4.1. Oxidative Stress (ROS/NOS): Aberrant Mitochondrial-Derived ROS/NOS Leakage In T2DM End-Organ Complications

\section{Major Existing And Emerging Hypotheses for LOAD}

5.1. Endothelial Cell Activation and Impaired Cerebral Blood Flow in Diabetic db/db Models

6. Neuropathologic Changes in LOAD

7. Discussion and Concluding Remarks

7.1. Metabolic Hypothesis: Hyperglycemia and Hyperinsulinemia

7.2. The Antimicrobial Protection Hypothesis: Antimicrobial peptide(s) (AMP)

\section{Creative commons act statement}

This review contains images that are available by the creative commons act 4.0. When images are used in this review that have been previously published by author or other authors the term CC BY 4.0 will be utilized in the legends with appropriate references provided. Presented herein, is the address to the creative commons act website. http://creativecommons.org/licenses/by/4.0/ accessed July 10, 2019.

\section{Introduction}

The human central nervous system culminates in the brain that contains a virtual galaxy of cells within a universe. Estimates of brain cell numbers includes: 100 billion neurons [1], 85-100 billion glia (astrocytes, microglia and oligodendrocytes) [2] and 0.15 quadrillion synapses [3], which makes our brain cell numbers somewhat comparable to that of our own Milky Way galaxy. Additionally, there are vascular cells coursing throughout the brain consisting of endothelial cell(s) (EC), pericyte(s) (Pcs) and vascular smooth muscles cell(s) (VSMC)). Each of these cells have multiple unique identifying ultrastructural form's and function's [4-9]. This review focuses on the capillary neurovascular unit(s) (NVU), which are a dense three-dimensional mesh-like network of microvessels that insures proper nutrient delivery (specifically oxygen and glucose) and nutrient exchange of metabolic byproducts from glia and neuronal cells to the systemic circulation. Importantly, the NVUs provide the necessary cerebral blood flow to allow for functional hyperemia of active neurons. It has been estimated that there are approximately 100 million capillary NVUs that course through the brain for approximately 400 miles, which also allow for a vast surface area of approximately 20 square meters in order to provide molecular transport to and from the systemic circulation [10].

The human brain basic organizing principles are centralization within a highly protected exoskeleton (the skull) with vast integration and regulation. The brain gathers information from the outside world via its five primary senses including sight, sound, smell, taste and touch and inside the body from the peripheral nervous system (peripheral neurons, spinal cord) and the brain itself (including the ganglia, mid and hind brain). These highly coordinated functions of the brain are based on genetic inheritance - epigenetic and environmental cues, which result in each of us having a unique individual 'brain print' similar to each of our unique fingerprints.

There is really no way that words can totally describe or express all of the possible permutations and computations that our brain is capable of making at any given point of time. The brain is virtual continuous sensory in-put receiving, thinking-processing and decision-making galaxy of cells and connections in a constant environment of neuroelectric (plasma membrane) and neuromolecular (neurotransmitters - neurochemical messengers via synaptic vesicles) with transmissional flux into each synaptic cleft between neurons.

This review examines how type 2 diabetes mellitus (T2DM) increases the risk of impaired cognition, metabolic cognopathy (MC), diabetic cognopathy (DC) and late-onset Alzheimer's disease 
- dementia (LOAD) and/or mixed dementias (MD). Understanding how these two disparate disease mechanisms may intersect as a continuum of progression with aging along with genetic, epigenetic and environmental conditions are essential since T2DM and LOAD are occurring in parallel and pandemic proportions. A better understanding of how these two diseases with very similar risk factors intersect is especially important since they are both age-related diseases. Additional focus is given to the microcirculatory capillary NVU with its multiple barriers and how this ultrastructural and functional unit correlates to the brain's cerebral blood flow (CBF). NVU coupling is essential in order to provide oxygenation, nutritional, ion supply, waste removal of metabolic byproducts and importantly functional hyperemia in those regions where there is increased activity of neurons [4-9]. Thus, the brain and its vascular supply are the structural and functional substrate of the mind. Further, those individual's with T2DM who develop diabetic cognopathy and/or LOAD may become lost and locked within the confines of their own mind.

\subsection{T2DM, Late -onset Alzheimer's Disease-Dementia (LOAD) and Societal Aging}

T2DM is a heterogeneous, multifactorial, polygenic, endocrine, metabolic, chronic and agerelated disease characterized by obesity, insulin resistance (IR) and hyperglycemia. Further, T2DM is a result of the relative or complete impairment of insulin actions, signaling, and associated with a progressive decline in pancreatic beta cell function $[4,5,6,7,8,9,11,12,13,14,15,16]$.

We are now living in a period of time where there exists one of oldest-living global populations [16] and currently there is evidence that suggests the number of older people in our global population is going to increase even further in the coming decades (Fig. 1) [17].

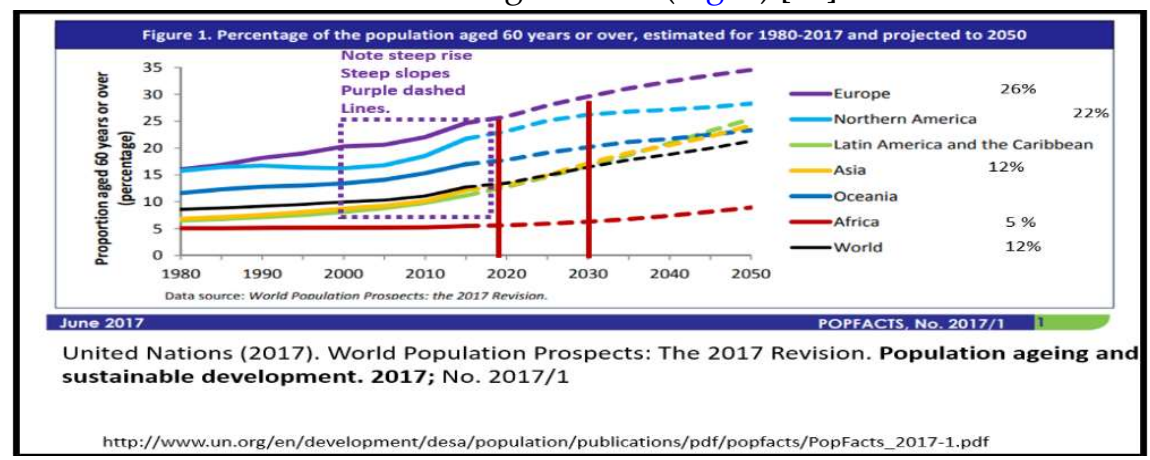

Figure 1. Graphical representation of the percent of the global population age 60 or greater. This figure is divided into global regions and age groups from United Nations (2017) population aging and sustainable development https://www.un.org/en/development/desa/population/publications/pdf/popfacts/PopFacts_20171.pdf accessed July 10, 2019.

This aging population is largely due to the aging baby boom (boomer) generation that benefited from the advancement of modern medicine including immunizations, antibiotics and cerebrovascular - cardiovascular care including hypertension and cholesterol control, coronary artery stenting; bypass grafting and stroke care. Additionally, the global aging boomer generation resulted from the high birth rates following World War II and is partially responsible for driving this pandemic. As the boomer generation continues to age, they also undergo an increase in age-related loss of skeletal muscle mass (extreme cases known as sarcopenia, moreso in males) with increasing skeletal muscle insulin resistance. As the global boomer generation ages there is an associated decrease in physical activity and increased consumption of compact calorie-dense diets currently known as the Western diet and contribute to the parallel pandemics of obesity, T2DM and LOAD. Globally, there are 425 million patients estimated to have T2DM, and by 2040, it is expected that this figure will rise to 642 million [18, 19, 20]. Importantly, it has been recently estimated that as the boomer generation ages, 13.5 million individuals just in the United States will manifest LOAD by the year 2050 (https://www.alz.org/help-support/resources/publications/trajectory_report accessed July 
10,2019 ) and the current lifetime risk of a 65 -year-old individual for LOAD is now estimated at $10.5 \%$ [21].

\subsection{Aging, Obesity, Insulin Resistance, Metabolic Syndrome and the H and E Phenomenon}

The current societal aging phenomenon as presented in section 1.1 is associated with excesses and these excesses have been incorporated into a syndrome that the late Gerald M Reaven initially termed Syndrome X (Fig. 2) [22], which was later termed the now familiar metabolic syndrome (MetS) or the " $\mathrm{H}$ " phenomenon ( $\mathrm{H}=$ hyper) and the " $\mathrm{E}$ " phenomenon ( $\mathrm{E}=$ excesses) (Fig. 3). MetS and the $\mathrm{H} \& \mathrm{E}$ phenomenon may be defined as a clustering or constellation of metabolic factors and clinical syndromes, which are intimately linked together by insulin resistance and known to increase the risk of not only cerebro-cardiovascular (CVD) disease and T2DM but also may link age-related LOAD. Importantly, obesity (specifically visceral obesity) is felt to be the initiating driver and insulin resistance is felt to be the linking factor of each of the four arms of the insulin resistance syndrome $X$ and the $\mathrm{H}$ and $\mathrm{E}$ phenomenon (Fig. 2, 3). The ATP III guidelines provide the proper parameters to identify those individuals with the MetS as follows: three of following five factors need to be confirmed: central obesity ( $\geq 94 \mathrm{~cm}$ (for males), $80 \mathrm{~cm}$ (females)), triglycerides concentration $\geq 150$ $\mathrm{mg} / \mathrm{dl}$, HDL cholesterol concentration $<40 \mathrm{mg} / \mathrm{dl}$ (males), $<50 \mathrm{mg} / \mathrm{dl}$ (females), values of blood pressure $\geq 130 / 85 \mathrm{~mm} \mathrm{Hg}$ and glycemia $\geq 100 \mathrm{mg} / \mathrm{dl}$ [23].

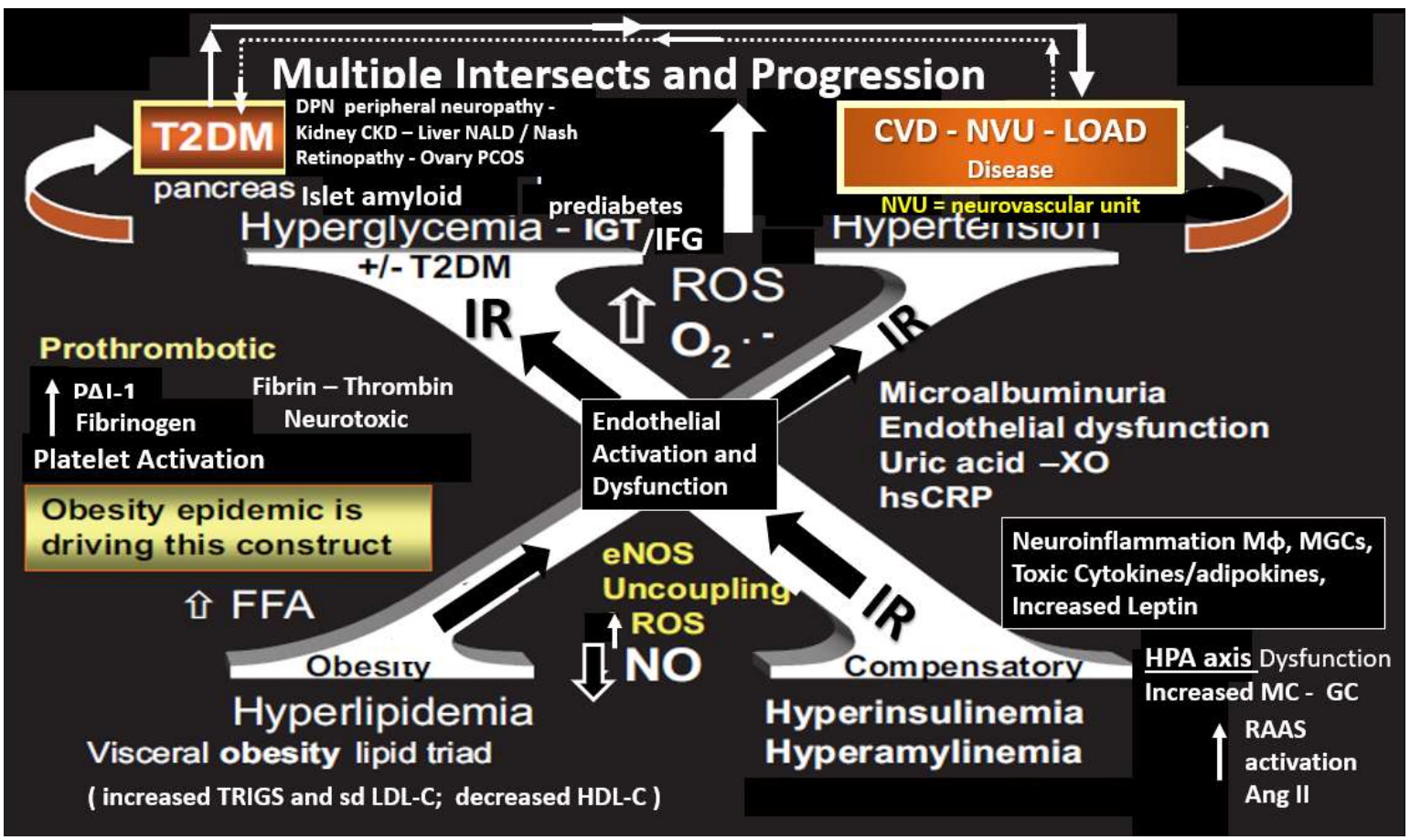

Figure 2. Metabolic syndrome with multiple intersects and progression. This image illustrates the Metabolic syndrome utilizing the letter " $X$ " in order to honor Reaven's original term Syndrome $X$ who championed the concept that resistance to insulin-mediated glucose disposal was a characteristic of patients with T2DM, which was later termed the Metabolic syndrome (MetS). Note the four different hyper-conditions associated with the MetS on each arm of the X. This figure depicts peripheral IR; however, there may be a contribution to central brain insulin resistance (BIR). CKD = chronic kidney disease; $C V D=$ cardio-cerebrovascular disease; DPN = diabetic peripheral neuropathy; eNOS = endothelial nitric oxide synthase;ET-1= endothelin 1; GC = glucocorticoid; IR = insulin resistance; FFA = free fatty acids; hs CRP = highly sensitive $C$-reactive protein; IGT = impaired glucose tolerance; IFG = impaired fasting glucose; LOAD = late onset Alzheimer's disease; $M G C=$ Microglial cell $; M \phi=$ macrophage; $M C=$ mineralocorticoid $; N O=$ nitric oxide; $N A L D=$ non-alcoholic liver disease; NASH = non-alcoholic steatohepatitis; O2- = superoxide; PCOS = 
polycystic ovary syndrome; T2DM = type 2 diabetes mellitus; $R A A S=$ renin angiotensin aldosterone system; ROS = reactive oxygen species; TNF $\alpha=$ tumor necrosis alpha; XO =xanthine oxidase.

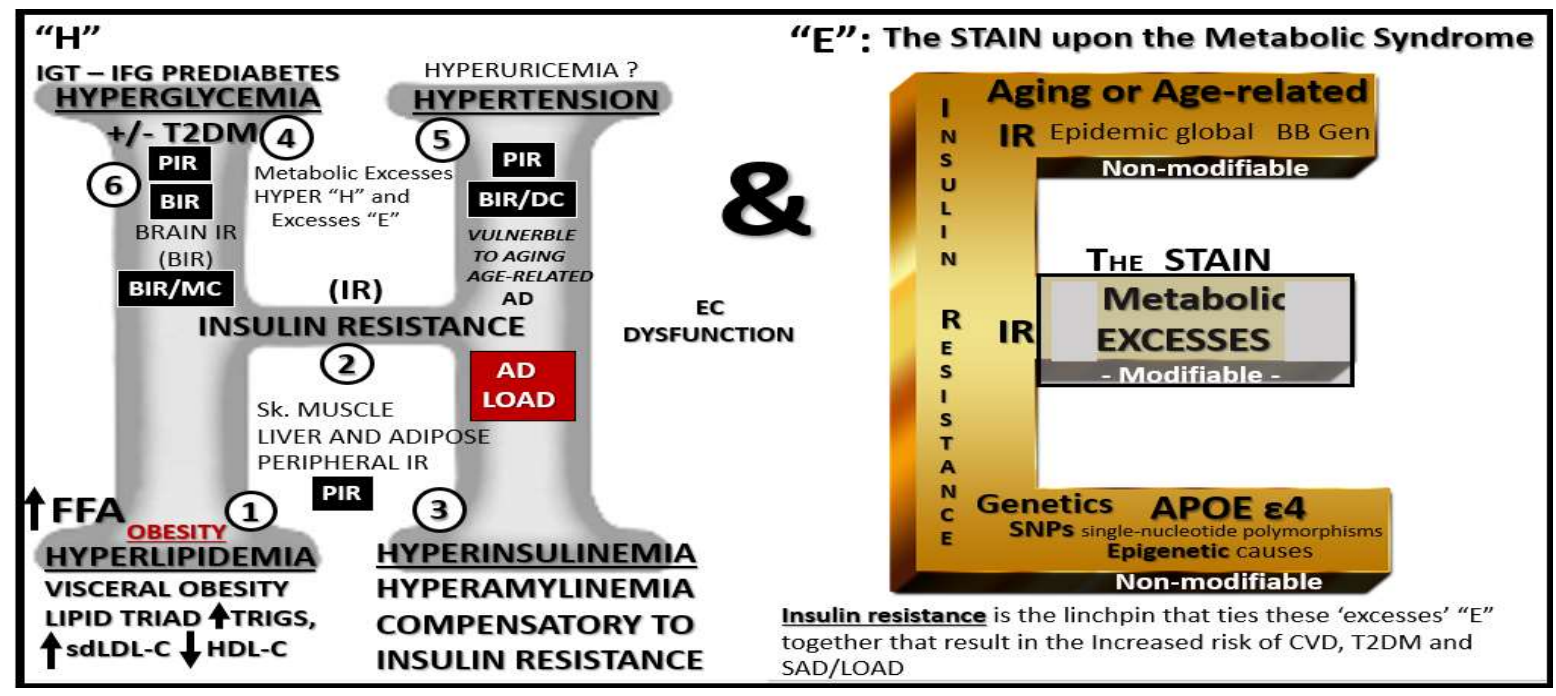

Figure 3. " $\mathrm{H}$ " and " $\mathrm{E}$ " hyper and excess phenomenon stain upon the metabolic syndrome. The " $\mathrm{H}$ " phenomenon represents the numerous HYPER-metabolic/clinical abnormalities associated with the metabolic syndrome in Fig. 2 and represents hyperlipidemia, hyperinsulinemia - hyeramylinemia, hyperglycemia, hypertension and the emerging hyperuricemia hypothesis. Furthermore, it illustrates at least six steps in the progression of this process that may be responsible for T2DM progression and increased vulnerability to the development of late onset Alzheimer's disease (LOAD). Note how insulin resistance (IR), step two, provides a linchpin to link the arms of the $\mathrm{H}$ and the metabolic stain upon T2DM and LOAD. Additionally, the "E" represents the excesses, which also may be associated with the metabolic syndrome. $A P O E \varepsilon 4=$ apolipoprotein $E$ epsilon $4 ; B I R=$ brain insulin resistance; $B B$ Gen = baby boomer generation; $E C=$ endothelial cell; HDL-C = high density lipoprotein cholesterol; IFG = impaired fasting glucose; IGT = impaired glucose tolerance; $P I R=$ peripheral insulin resistance; $S k .=$ skeletal; $S N P S=$ single nucleotide polypeptides Trigs = triglycerides; sdLDL-C = small dense low-density lipoprotein cholesterol.

\subsubsection{Insulin Resistance: Peripheral and Central-Brain Insulin Resistance}

It is difficult to sort out the differences of peripheral insulin resistance (PIR) and hyperglycemia - glucotoxicity, since they are both present in T2DM and in brain insulin resistance (BIR) in LOAD (Box 1). 
Difficulty In Sorting Out The Contributions Of Insulin Resistance And Hyperglycemia In T2DM And LOAD

Hyperinsulinemia - Peripheral Insulin Resistance (PIR)
Met S, Prediabetes and T2DM contribution to brain insulin
resistance (BIR): BIR thought to be associated with LOAD

I. Decreases Insulin Receptors at EC of the NVU impaired insulin uptake.

II. Excess insulin competes with $A \beta$ degradation at IDE at EC of NVU resulting in decreased clearance - efflux of $A \beta$.

III. Impairs canonical insulin signaling in peripheral cells. and brain cells - Brain insulin resistance (BIR) in neurons, which may contribute to increased $A \beta$ fibril production; tau hyperphosphorylation with PHF and NFT.

IV. May inhibit insulin dependent flow mediated vasodilation. Thus, endothelial cell dysfunction.

V. Contributes to impaired macro-autophagy - mitophagy and disrupts the Lysosomal degradation pathway.

VI. $A \beta$ increases in LOAD may induce Insulin resistance in neurons.

VII. Age-related decrease in insulin sensitivity and insulin resistance.

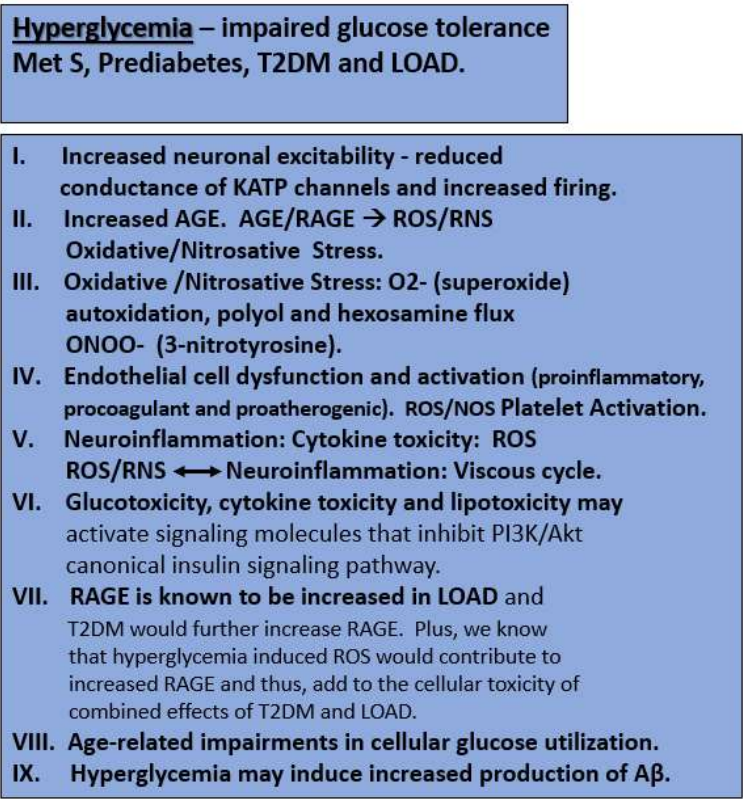

Box 1. Difficulty in sorting out the contributions of insulin resistance and hyperglycemia in T2DM and LOAD. Insulin resistance and hyperglycemia are both important in the development of the endorgan complications associated with T2DM including the brain. It is very difficult to sort out the individual roles of hyperinsulinemia due to peripheral insulin resistance (PIR) in type 2 diabetes and central brain insulin resistance (BIR) in LOAD as well as hyperglycemia in T2DM and LOAD. What makes this so difficult is that T2DM has both a singular early insulin resistance state that compensates and initially protects individuals from the development of hyperglycemia but not without a price to pay (due to the damaging effects of hyperinsulinemia) not only in peripheral tissues but also in the brain followed over time and aging with ongoing insulin resistance. Hyperglycemia eventually evolves due to the inability of the pancreatic islet beta cells to compensate (islet beta cell exhaustiondysfunction and/or loss due to apoptosis) with ensuing hyperglycemia. This box attempts to outline what is currently thought to be known regarding insulin resistance and hyperinsulinemia and compare these effects to hyperglycemia in the brain that may contribute to the development of LOAD. $\mathrm{A} \beta=$ amyloid beta; $\mathrm{AGE}=$ advanced glycation end-products; $\mathrm{BIR}=$ brain insulin resistance; $\mathrm{EC}=$ endothelial cells; IDE = insulin degrading enzyme; KATP = Adenosine triphosphate-sensitive potassium channel; LOAD = late-onset Alzheimer's disease; MetS = metabolic syndrome; NVU = neurovascular unit; O2- = superoxide; PHF = paired helical fragments; PIR = peripheral insulin resistance; NFT = neurofibrillary tangles; RAGE = receptor for advanced glycation end-products; RNS = reactive nitrogen species; $\mathrm{ROS}=$ reactive oxygen species; $\mathrm{T} 2 \mathrm{DM}=$ type 2 diabetes mellitus .

Further, the effect PIR and glucotoxicity may have some important bearings on the development of brain insulin resistance (BIR) in LOAD. For example, Ferreira LSS et al. [24] utilize the concept of combined FFA toxicity due to accumulation of visceral obesity and PIR and hyperglycemia, which results in AGE toxicity to induce brain inflammation and BIR in obesity and T2DM via a cascade of events as follows: Visceral omental obesity induces excessive toxic free fatty acids and hyperglycemia induces advanced glycation end-products (AGE) to result in blood-brain barrier (BBB) permeabilization followed by brain neuroglia (astrocyte and microglia) inflammation with the production of a host of toxic cytokines to induce neuronal BIR and endoplasmic reticulum stress with synaptic dysfunction and/or loss and its association with neuronal dysfunction and neurodegeneration in age-related LOAD. However, it is currently becoming more and more evident that there exists a primary BIR in LOAD [25-29].

\subsection{Hypothalamic-Pituitary-Adrenal (HPA) Axis Dysfunction in the Metabolic Syndrome H and E Phenomenon}


The HPA axis in homeostatic conditions is a tightly regulated system that represents one of the body's response mechanisms to acute and chronic physiological or psychological stress. HPA axis dysfunction is manifest in obesity, insulin resistance, MetS and T2DM as excesses "E" of mineralocorticoids (aldosterone), glucocorticoids (corticosterone in rodents and cortisol in humans) and may be associated with increased sympathetic tone [8, 30]. However, in diet induced obesity (DIO) Western models and preclinical diabetic $\mathrm{db} / \mathrm{db}$ models and humans it is observed to become dysregulated - HPA axis dysfunction. Elevated levels of aldosterone and corticosterone contribute to insulin resistance and cardiovascular disease and may even implicate the cerebral arteriole and capillary NVUs.

Previously, in DIO Western model cohorts the adrenal gland underwent considerable ultrastructural remodeling (unreported data) [8]. This included adrenal cortical hyperplasia and hypertrophy in zona glomerulosa and zona fasciculata, which may result in increased aldosterone and corticosterone with markedly dilated capillaries within both cortical and medullary regions suggesting increased adrenal blood flow (Fig. 4, 5) and also may be associated with adrenal medullary catecholamine output into the peripheral systemic circulation with increased sympathetic vascular tone (however in these models we did not demonstrate an increase in blood pressure at 8 , 12,16 or 20 -weeks of observation). Importantly, the above abnormalities in the Western models strongly suggested the presence of inappropriate and impaired HPA feedback control with dysregulation between these neuroendocrine and systemic endocrine organs. These observations and findings in the Western model were in agreement with another study of the adrenal gland [31].

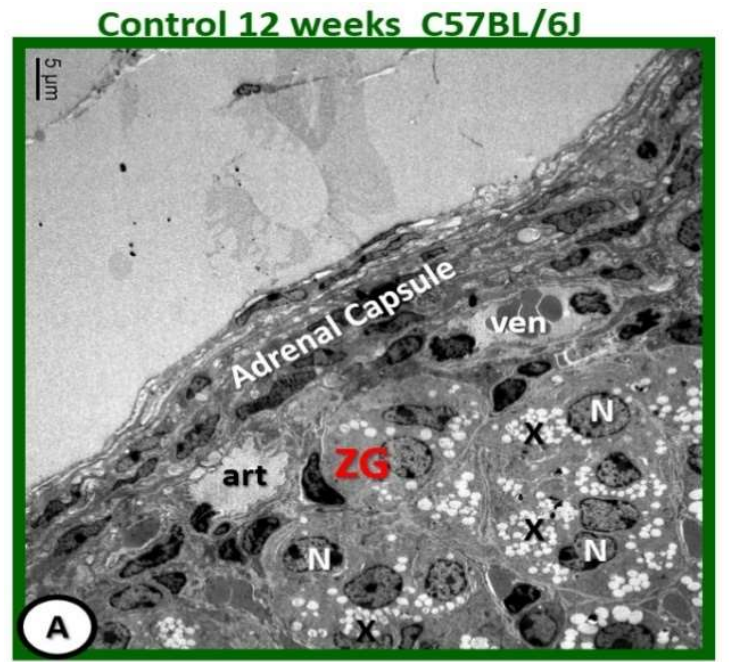

Normal House Chow diet

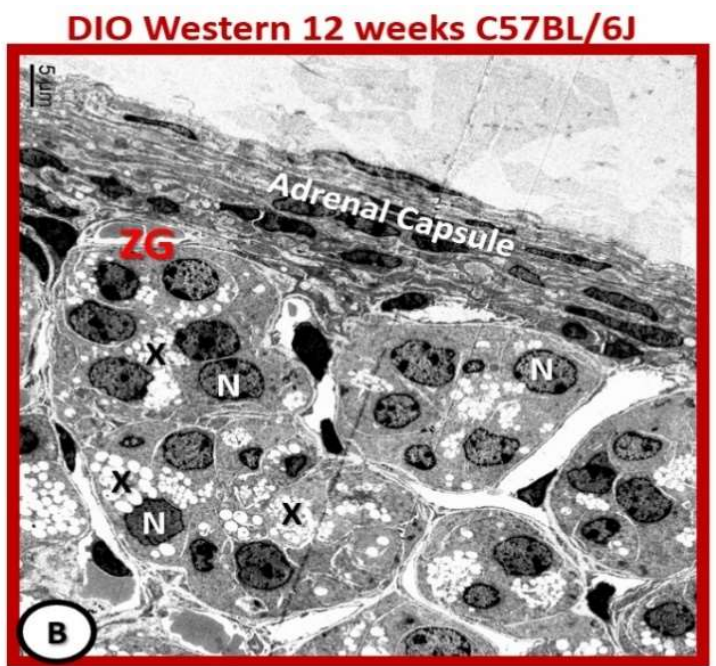

High fat, high sucrose and fructose DIO Obese "Western" model.

Figure 4. Adrenal gland zona glomerulosa hypertrophy and hyperplasia with capillary dilation in diet induced obesity Western models. Panel A illustrates the normal control zona glomerulosa (ZG). Panel B depicts the abnormal remodeling changes found in the ZG cells containing spherical secretory granules (electron lucent) that contain aldosterone $(X)$. Note the hyperplasia and hypertrophy and dilated capillaries as compared to the control models in panel A. Magnification X300; scale bar = 5 $\mu \mathrm{m} . \quad$ art $=$ arteriole; $N=$ zona glomerulosa cell nucleus; ven = venule; $X=$ electron lucent spheres of zona glomerulosa secretory granules; ZG = zona glomerulosa cells. 


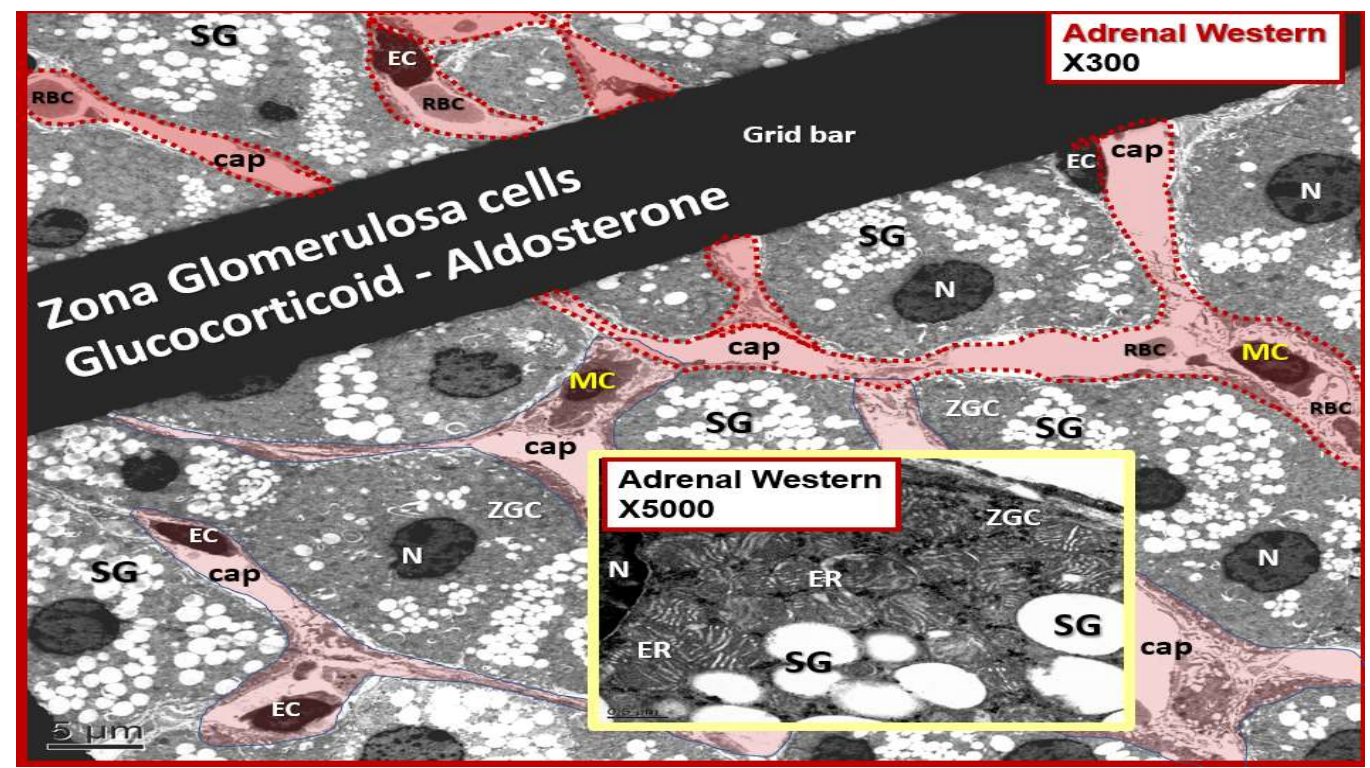

Figure 5. Dilated capillaries bathing the zona glomerulosa cells in Western models. This image depicts the dilated capillaries (cap; pseudo-colored red) that appear to be continuously bathing the zona glomerulosa cells (ZGCs) and nuclei (N). Insert emphasizes the zona glomerulosa secretory granules (SG) and the extensive endoplasmic reticulum (ER). Magnification x300; scale bar $=5 \mu \mathrm{m}$. Insert magnification X5000; bar $=0.5 \mu \mathrm{m}$. EC = capillary endothelial cell(s); $M C=$ mononuclear cell(s); $R B C=$ red blood cells.

In addition to the HPA axis dysfunction it is thought that the visceral-omental adipose tissue excess is associated with an increased compressive effects on the kidney that may result in an increase in the renin-angiotensin-aldosterone system (RAAS). Also, the excess aldosterone may also be associated with a visceral adipose derived aldosterone releasing factor [32, 33, 34].

\section{Multiple Intersects Between Type 2 Diabetes Mellitus and Late-Onset Alzheimer's Disease}

LOAD, like T2DM, is a chronic age-related disease with a long preclinical or prodromal phase (up to 10 years which is somewhat similar to prediabetes - impaired glucose tolerance and impaired fasting glucose). Once diagnosed, LOAD may have a clinical duration of 8-10 years. Furthermore, in age groups over the age of 65 LOAD has a prevalence (10-30\%) and incidence (1-3\%) with a current lifetime risk of LOAD of approximately 10.5\%. [35].

The MetS and $\mathrm{H}$ and $\mathrm{E}$ phenomenon coupled with aging allow for multiple intersects between T2DM and LOAD with IR as the possible linking linchpin between these two age-related and pandemic diseases (Box 2). 


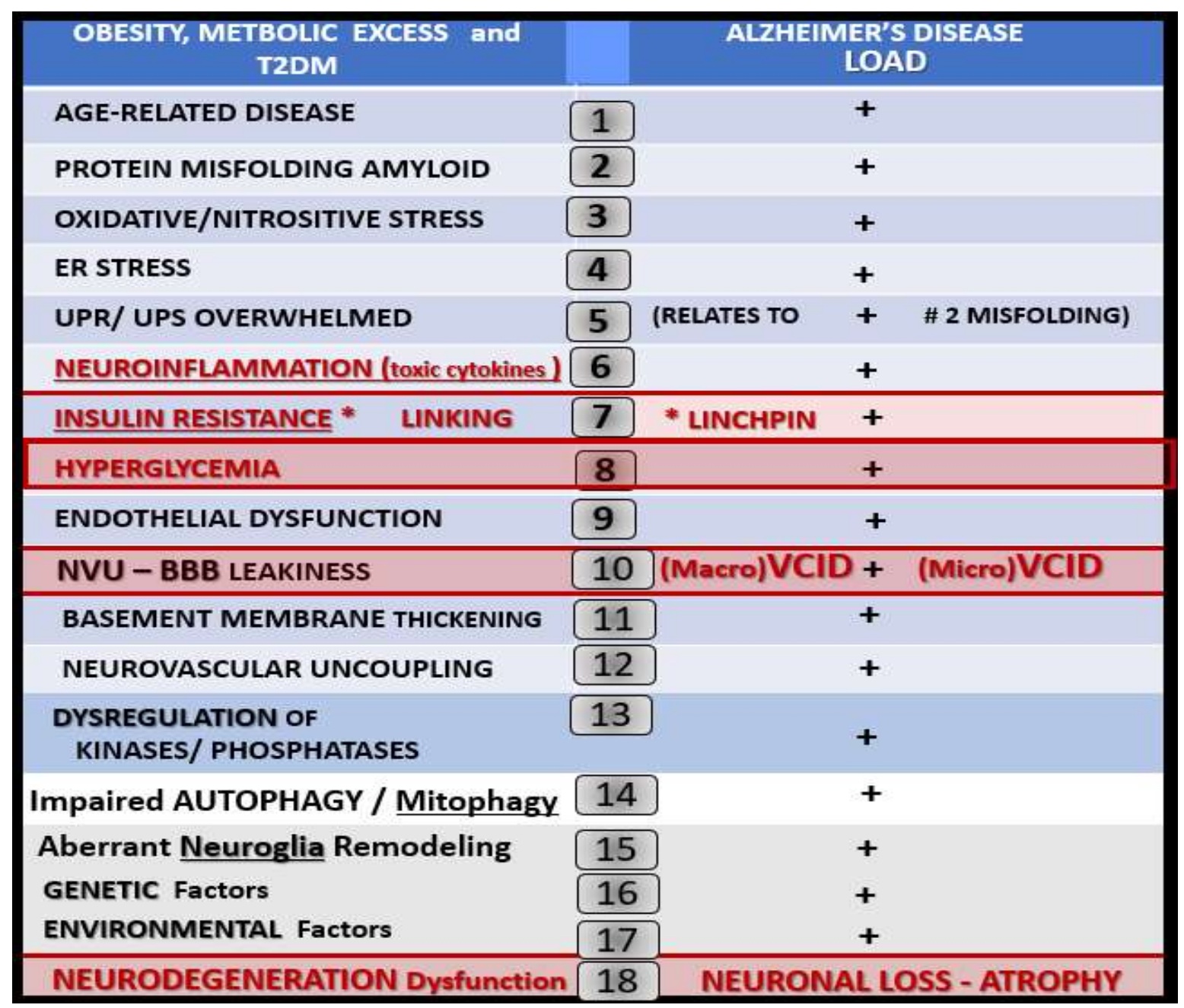

Box 2. Multiple intersects between T2DM and late-onset Alzheimer's disease. This figure illustrates the multiple (at least 18) intersects between T2DM and late onset Alzheimer's disease (LOAD). These multiple intersects contribute to the increase of LOAD in those individuals with T2DM. Importantly, note that insulin degrading enzyme (IDE) is related to \#7 Insulin Resistance and hyperinsulinemia in that elevated insulin of IR could compete with amyloid beta for degradation by the IDE and that advanced glycation end-products (AGE) and its receptor RAGE are important to \# 8 hyperglycemia in generating reactive oxygen species and AGE could also compete with RAGE for amyloid beta degradation. $A D=$ Alzheimer's disease; $B B B=$ blood-brain barrier; $E R=$ endoplasmic reticulum; $L O A D=$ late-onset $A D ; N V U=$ neurovascular unit; T2DM = type 2 diabetes mellitus; UPR/UPS = unfolded protein response.

Both T2DM and LOAD are known to have multifactorial risk profiles and therefore, it is likely that the relationship between these two diseases have multiple intersects and multifactorial risks that promote cognitive abnormalities and interact during the development of age-related LOAD with underlying T2DM.

The above converging global situations in 1.1. and 1.2. gives rise to great concern, in that, T2DM has recently been demonstrated to increase the risk of developing other chronic age-related diseases such as LOAD [36-47]. LOAD is responsible for $90-95 \%$ of all dementias. This is in contrast to early onset familial Alzheimer's disease (EOFAD), which develops prior to age 65 and may be related to specific genetic defects such as those in the amyloid precursor protein or presenilin genes and contributes to only approximately $4-5 \%$ of dementias [48]. Furthermore, there may be progression or a continuum of progression from obesity, metabolic syndrome, metabolic cognopathy, T2DM and diabetic cognopathy (DC) to vascular dementia (VaD), LOAD and mixed dementia (MD). 
Importantly, there has been a recent trend to soften the once hard-fixed clinical and histopathologic boundary-lines drawn between $\mathrm{VaD}$ and LOAD. Additionally, LOAD may now be considered to be under the umbrella of mixed dementia's (MD) (Fig. 6).

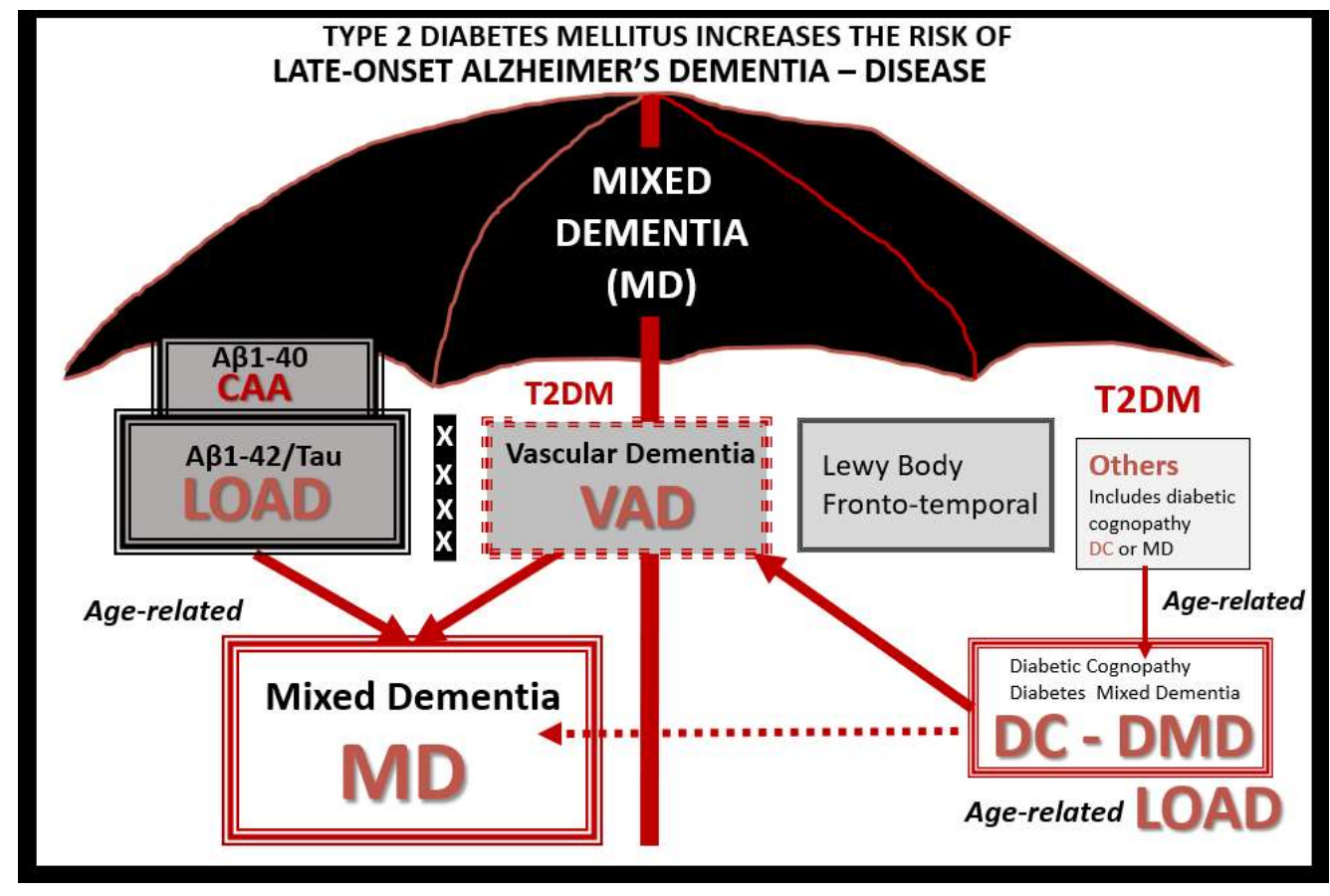

Figure 6. The overarching umbrella of mixed dementia and diabetic mixed dementia. Late-onset Alzheimer's disease (LOAD) is by far the most common of all dementias - $90-95 \%$ with early-onset Alzheimer's disease contributing approximately $4-5 \%$. What has been previously thought of as distinct dementias with rigid separating lines may now be thought of as belonging under the overarching umbrella of mixed dementia(s) (MD). Individuals who are clinically diagnosed with LOAD, to best of the clinician's ability, during life, are frequently found have a mixed dementia at the time of autopsy and neuropathologic examination. The histopathology consists of not only amyloid beta extracellular neuritic plaques, microtubule associated tau paired helical fragments and neurofibrillary tangles but also cerebral amyloid angiopathy (CAA) and evidence of microvascular capillary neurovascular unit (NVU) and arteriolar abnormalities in structure and function. Interestingly it is now being found that NVU dysfunction may precede the deposition of amyloid beta and tau in LOAD. Note the $X^{\prime}$ 's within the thickened bar (representing a softening) that divides LOAD from vascular dementia $(\mathrm{VaD})$ and the other dementias under this overarching umbrella. The others box in the figure (far right) may include the following: Metabolic e.g. diabetic cognopathy or diabetic mixed dementia (DMD), trauma, infection, Parkinson's disease, alcohol, rare genetic causes, druginduced and depression-related causes of dementia.

This fading phenomenon of the demarcation between $\mathrm{VaD}$ and LOAD is largely due to the findings of mixed dementia at the time of autopsy and the early finding of neurovascular microvascular disease in AD (Fig. 7) [49-51]. The findings of mixed dementia consisting of LOAD (neuritic $\mathrm{A} \beta$ plaques and tau neurofibrillary paired helical fragments (PHF) and neurofibrillary tangles (NFT), cerebral amyloid angiopathy (CAA) and $\mathrm{VaD}$ are being found to co-exist more and more when post-mortem tissues are examined by neuropathologists providing their mixed histopathologic findings, which suggests strongly that LOAD may be a spectrum disorder or disease (Fig. 7). 

(LOAD) AT EITHER ENDS OF THE SPECTRUM WITH MIXED DEMENTIA'S PREVAILING AT THE NEUROPATHOLOGICAL LEVEL

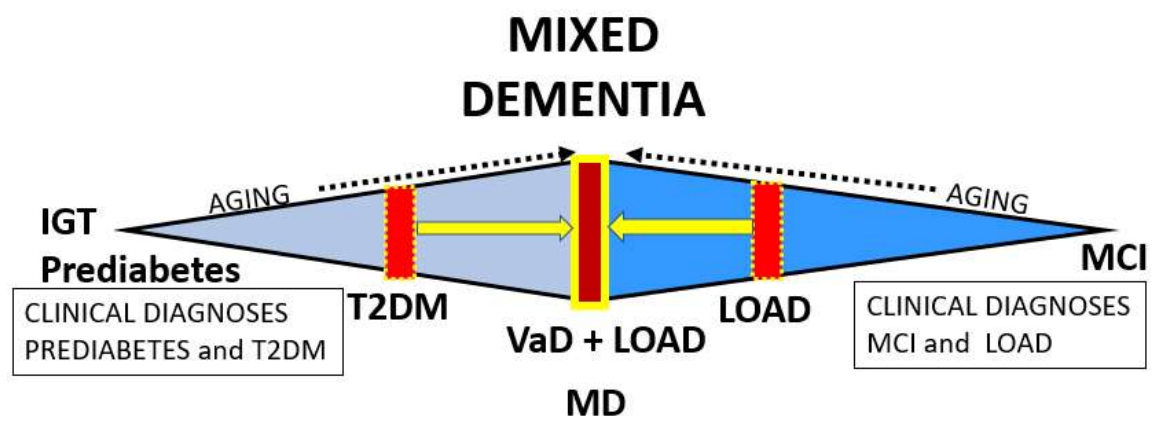

Figure 7. LOAD as a spectrum disease. Late-onset Alzheimer's disease (LOAD) and vascular dementia (VaD) are the two most common dementia's in older individuals with LOAD being the most common. However, when brains are examined in those patients clinically diagnosed as LOAD it is found by autopsy and neuropathological examination that most have a mixed dementia (MD) of LOAD, cerebral amyloid angiopathy (CAA) and VaD making them mixed (co-occurrence) dementia's (MD). Therefore, MDs could or should now be considered the most common form of dementia in older adults age 65 and older.

Also, recently recognized community-based neuropathologic studies have shown that there may be many different complex constellations of underlying pathologies, which may lead to cognitive decline in LOAD [52]. Mixed dementia's equals co-occurrence dementia's for example LOAD + VaD $+\mathrm{CAA}=$ mixed dementia when we include neuropathologic findings of post-mortem autopsy. Furthermore, it has been recently shared that neurovascular disease, particularly microvascular NVU disease (vascular contributions of impaired cognition and dementia (VCID - microvascular, Micro-VCID), may be one of the earliest findings in LOAD [53, 54]. Also, the 2-hit vascular hypothesis has been placed into acceptance (in addition to other multiple LOAD hypotheses) $[55,56]$ and recently the paper with 69 authors, which discussed in detail the importance of the NVU and its constituent cells as well as other related risk factors [57]. Microvascular NVU dysfunction, cellular remodeling and NVU uncoupling may result in hypoperfusion and decreased CBF (discussed in greater detail in section 5.1.), which appear to be early markers of neurodegeneration. These remodeling changes may set in motion a serious aberrant cascade of remodeling and functional events in the brain that may be concurrent or even precede the synaptic dysfunction and deposition of neuritic plaques or neurofibrillary tangles and result in the cognitive decline of age-related LOAD neurodegeneration. It is commonly known that T2DM increases the risk for $\mathrm{VaD}$ and a recent study of 28 prospective observational studies in 2012, which included 89,708 diabetic patients demonstrated a $73 \%$ increased risk of all type dementias (which included LOAD), a 56\% increase of LOAD and a $127 \%$ increase of $\mathrm{VaD}$ in diabetic human patients [58]. However, it might be very interesting if one would consider LOAD as a mixed dementia (inclusive of $\mathrm{VaD}$ and LOAD) in regards to how these percentages might remarkably change.

\section{Continuum of Progression: From Obesity, Insulin Resistance to T2DM to LOAD}

There appears to be a continuum of progression in the natural history of T2DM inclusive of MetS and obesity to LOAD. From obesity (specifically visceral or omental) to MetS to T2DM and the multiple intersects and increasing cellular vulnerability to the development of LOAD and mixed dementia(s) (MD) (Fig. 8).

\subsection{Obesity}


As mentioned earlier in section 1.2, obesity seems to be the driver of both the Hyper " $\mathrm{H}$ " and "E" phenomenon and the Met S. (Fig.2,3) with associated metabolic cognopathy and minimal cognitive impairment (MCI) to T2DM and diabetic cognopathy (DC) to NVU remodeling, neuroinflammation, neurodegeneration and LOAD. Peripheral insulin resistance (PIR) and central brain insulin resistance (BIR) and impaired insulin signaling seem to be the link between T2DM and LOAD. So it seems that T2DM may increase the vulnerability and the risk of LOAD via a progressive continuum: from visceral-omental mid-life overweight and obesity that is known to independently increase $\mathrm{LOAD}$ as well as $\mathrm{VaD}$ [59]. This progressive continuum to LOAD via the MetS and " $\mathrm{H}$ " and "E" phenomenon to T2DM seems to increase the risk of neurodegeneration via a 2-hit vascular hypothesis: VCID to LOAD (Fig. 8).

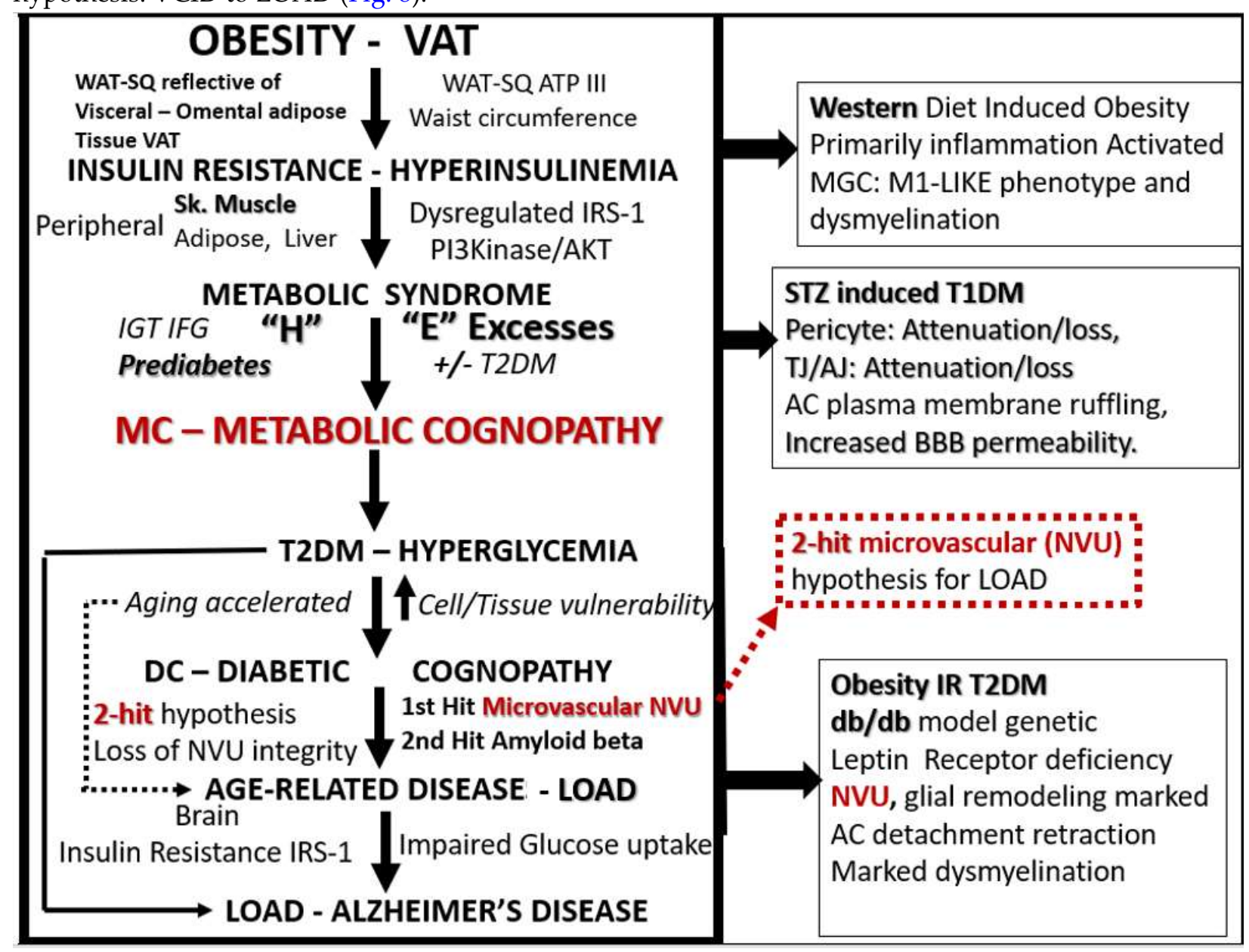

Figure 8. The progressive continuum from visceral obesity to type 2 diabetes mellitus to neurodegeneration and late-onset Alzheimer's disease (LOAD). This continuum of progression (downward arrows) may occur independently as independent risk factors or interact synergistically and concurrently over time (aging). This figure also represents the continuum of progressive metabolic excesses and stresses involved to advance to LOAD via obesity, insulin resistance, metabolic syndrome and the " $\mathrm{H}$ " and " $\mathrm{E}$ " phenomenon and a metabolic cognopathy to prediabetes and/or overt T2DM with glucotoxicity to accelerated aging and increased neurovascular unit, vascular mural cells, neuroglia, neuronal cellular vulnerability and remodeling to metabolic cognopathy, diabetic cognopathy and aging with the increased risk of neurodegeneration and LOAD. In this figure we propose that these above precursors are more of a continuum of progression from various stages, one to the next; however, some have posited that they could each represent separate pathologies that may be differentially expressed over time and thus, remain age-related. The boxes on the right side of this figure refer to our independent findings in the brains of the diet induced obesity, insulin resistant model with impaired glucose tolerance or prediabetes Western mouse model [8], the streptozotocin induced type 1 diabetic mouse model [9] and the $\mathrm{db} / \mathrm{db}$ mouse model of obesity, insulin resistance and T2DM [4-7]. Note the intersects between \#10 in Box 2 to the red dashed outline 
of the 2-hit microvascular hypothesis and vascular contributions to cognitive impairment and dementia (VCID). ATP III = adult treatment panel III of the National Cholesterol Education program; IFG = impaired fasting glucose; IGT = impaired glucose tolerance; IRS-1 = insulin receptor substrate-1; NVU = neurovascular unit; PI3kinase/ $A K T=$ phosphatidylinositol 3/protein kinase B; Sk= skeletal; $S Q=$ subcutaneous; $V A T=$ visceral adipose tissue; $W A T=$ white adipose.

Previous studies in obese-prediabetic models with impaired glucose tolerance have demonstrated that the diet-induced obesity Western model (with high fat, high sucrose and fructose in C57B6 models) were observed to have lipofuscin-like bodies within the interstitial spaces that were being avidly consumed by activated MGCs with some dysmyelination (markedly less electron density of myelin) of axons in layer III of the mid cortical grey matter. Overall, this model was primarily a neuroinflammatory model with activation and senescence of microglia without any NVU remodeling [8]. Our next model was not an obese model but a lean streptozotocin induced type 1 diabetes model, which demonstrated marked NVU remodeling with blood-brain barrier (BBB) disruption and neurovascular unit dysfunction with increased permeability and loss of NVU BBB integrity. In this model we deduced that glucotoxicity resulting in excessive oxidative stress was the primary cause of NVU remodeling, since treatment with a specific mitochondria carbonic anhydrase inhibitor protected these NVU remodeling changes as well as increased permeability [9]. Our most recent model studied was the obese, insulin resistant, T2DM female $d b / d b$ mouse model [47]. This model demonstrated marked NVU, microglia cell(s) (MGC) and oligodendrocyte(s) OL and myelin remodeling that was protected utilizing a 10-week treatment period with the glucose lowering anti-diabetic sodium glucose transporter 2 (SGLT2) inhibitor from 10 - 20 weeks of age (Fig. 8) [7]. In this diabetic obese $d b / d b$ model we were also to demonstrate a marked expansion in aortic visceral perivascular adipose tissue that contained hypertrophic unilocular adipocytes that were rupturing with the spillage of toxic proinflammatory free fatty acids (unpublished data). These remodeling changes were also associated with chronic inflammation due to peripheral systemic macrophage infiltration and thus provided for excessive toxic cytokines and adipokines to be available to the aortic wall and systemic circulation, which was associated with vascular stiffening. This vascular stiffening could increase abnormal pulsatile mechanical forces associated with NVU - microvascular structural damage and remodeling to the NVU of brain due to increased pulse wave velocity and increase the expansion via increased pulse pressure in addition to also being a source of chronic inflammation due to systemic cytokines-adipokines from the perivascular adipose tissue. Thus, the $d b / d b$ model [47] was able to 'fill-in' many of our gaps in knowledge between the diet-induced obesity Western model [8] and the type 1 streptozotocin induced diabetic models [9].

\subsection{Capillary Neurovascular Unit (NVU)}

The capillary NVU is a readily identifiable ultrastructural constant when viewing brain tissue with transmission electron microscopy (TEM) (Fig. 9) [4-7].

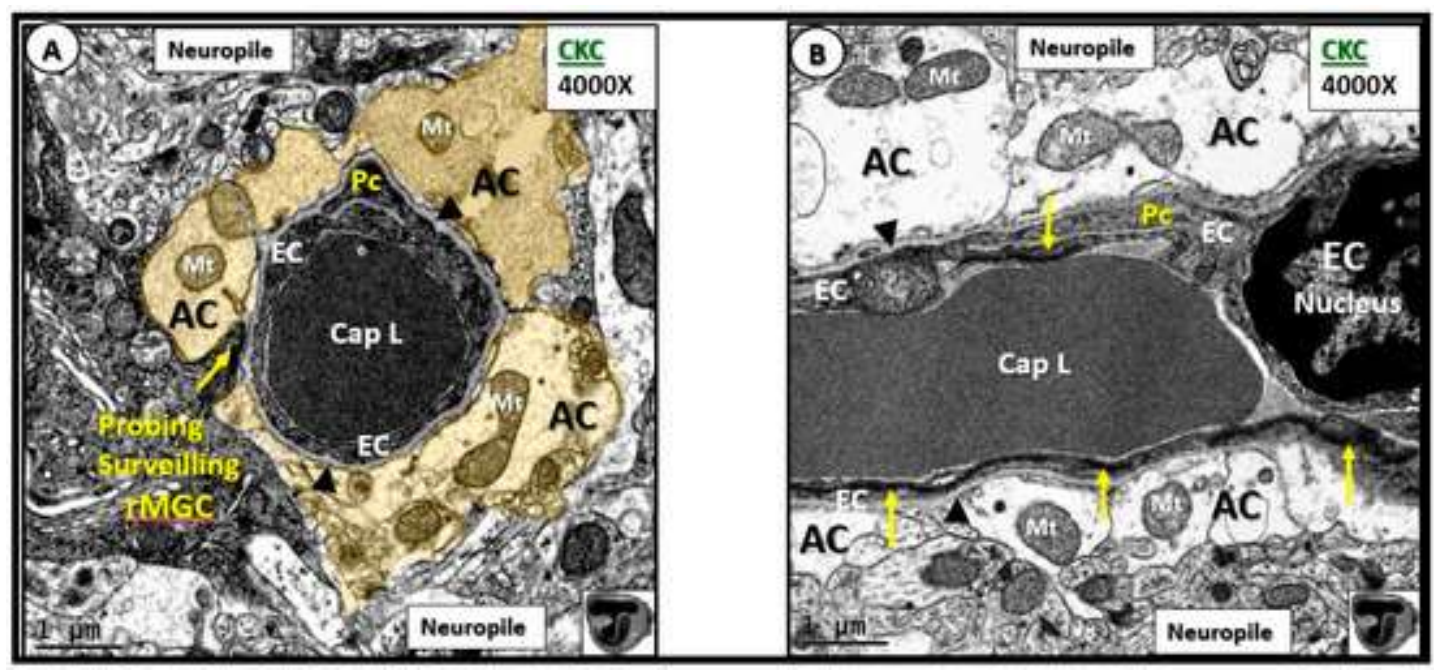


Figure 9. Normal neurovascular unit (NVU) morphology in control wild-type non-diabetic models (C57BL/KsJ; CKC control). Panels (A) (cross-section) and (B) (longitudinal section) illustrate the normal cellular ultrastructure of the NVU. Panel (A) depicts an electron-dense ramified microglia cell (rMGC) surveilling the NVU (arrow). The NVU capillary consists of an endothelial cell (EC) encircling a capillary lumen (Cap L) whose basement membrane (BM) splits (arrowheads) to encompass the pericyte (Pc) foot process. Note how the pseudo-colored golden astrocyte (AC) end-feet encompass and tightly abut the capillary EC and Pc BMs. Note that the AC clear zone in panel (A) was pseudocolored golden to emphasize its importance in the NVU, while it exists as a clear-zone with a reduced electron-dense cytoplasm as compared to other cells within the brain, and represents not only a golden halo, but also a clear zone or corona of AC foot processes surrounding the EC and Pc cells of the NVU (panel (B)). Panel (B) illustrates the electron-lucency of the AC clear zone halo or corona that tightly abuts and encircles the NVU EC and Pc BMs. Note the EC nucleus (far right side) and the highly electron-dense tight junctions/adherens junctions (TJ/AJ) complex (yellow arrows) that are not readily visible in panel (A) (arrows). Also, note that the mitochondria (Mt) have an electron-dense $\mathrm{Mt}$ matrix and that cristae may be noted even at this magnification. Note that the NVU is encompassed by the outermost abluminal neuropil (neuropil). In the bottom right-hand corner, note the logo of red blood cells within a capillary NVU that are in the shape of the letter T overlying the letter J, which are used to abbreviate tight junction(s). Magnification $\times 4000$; scale bar $=1 \mu \mathrm{m}$. Image available via license CC BY 4.0. [4].

This multicellular structural and functional unit in the brain consists of the following cells: ECs, Pcs, ACs, MGCs, OLs and neurons. Within the NVU is contained the EC paracellular BBB synthesized primarily by ECs and supported structurally and functionally by Pcs and additionally supported and maintained by a corona of surrounding-encircling AC foot processes (ACfp). The two primary cells of the capillary NVU are the luminal continuous monolayer of ECs and mural supportive Pcs, which are embedded within the EC basement membrane (BM) creating an inner and outer BM. This is in contrast to the arterial NVU, in which vascular smooth muscle cells provide for mural arteriole support with similar abutting of ACfp. The third contiguous cells belong to the multiple ACfps that tightly abut the EC and Pc outer BMs. Probing ramified microglia cells, oligodendrocytes and oligodendrocyte linage precursor cell(s) (OPC) (especially in subcortical and white matter regions) along with neuronal axons (unmyelinated and myelinated) may also be noted to be adjacent to the outer basement membrane of the NVU [4-7].

There are basically three to four barriers of the NVU from luminal to abluminal regions: $i$. endothelial glycocalyx (ecGCx); ii. endothelium (ECs) inclusive of BBB tight and adherens junctions (TJ/AJ) and the EC cytoplasm itself, which also utilizes transcytosis as a method of EC cellular transport; iii. EC and pericyte (Pc) and/or its pericyte foot processes (Pcfp) basement membrane (BM), which shares the common inner and outer basement membrane (BM); iv. astrocytes (ACfp) and their outer basal lamina. Of these three to four barriers, the BBB TJ/AJ that reside between EC cell-cell junctions of the NVU and between the blood and brain interstitial fluid provides the greatest trans-endothelial electrical resistance (TEER) control over the immediate microenvironment of brain cells. Additionally, there are other interfaces-barriers that are also important such as the choroid plexus epithelium between the blood and ventricular cerebrospinal fluid (CSF), and the arachnoid epithelium between the blood and subarachnoid CSF. It seems appropriate to discuss these cells and barriers individually from the inside luminal to outside abluminal regions of the NVU [4-9].

\subsubsection{Endothelial Cell(s) (EC) - Endothelium}

The endothelium is the continuous, epithelial, highly polarized, monolayer cell that lines the luminal surface of the NVU and serves as the interface between the peripheral circulating blood and its cellular, molecular proteins, ion constituents and the brain (Fig. 9). The NVU ECs are responsible for creating and maintaining the NVU permeability and lumen formation [60]. The EC is responsible for 2 initial barriers, which create the BBB consisting of TJ/AJ, junctional adherens molecules and cytoskeletal zona-occludin-1 and beta catenin's that are formed between adjacent endothelial cells [4- 
7] and the EC surface layer the endothelial glycocalyx (ecGCx). The apical luminal and basolateral abluminal polarity of the brain EC is of utmost importance in creating the normal requisite physiologic functions of the NVU in that many of its influx - efflux and specific transporters proteins require a specific location to be physiologically functional $[4,60]$. It is also important to note that while the integrity of the paracellular TJ/AJ proteins in ECs are of critical importance in EC permeability, there also exists an intracellular transcytotic route for increased EC transcellular transport and permeability mechanisms. In fact, our diabetic $d b / d b$ models have demonstrated that there exists not only an ultrastructural abnormality in TJ/AJ with attenuation and/or loss but also an increase in the EC transcytotic-pinocytotic vesicles plus other important remodeling change such as inflammation and basement membrane thickening [4, 7] (Fig. 10).

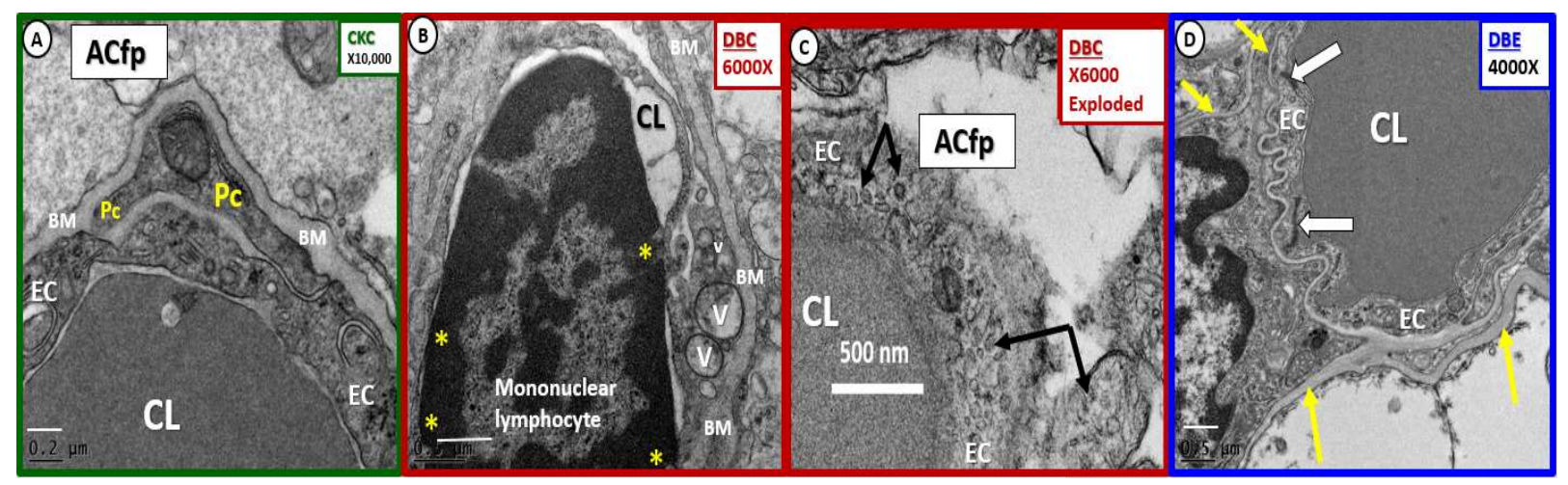

Figure 10. Multiple endothelial cell remodeling changes in diabetic $d b / d b$ models. Panel A Illustrates the normal endothelial cell (EC), pericyte $(\mathrm{Pc})$ and astrocyte foot process (ACfp). Note that the basement membrane appears almost as thick as in the known BM thickening in panel B; however, note that the magnification is higher $(x 10,000)$ and the scale bar is not as wide in panel A (200nm) as in panel B (500nm). Panel B depicts adherent mononuclear lymphocyte and also note EC vacuoles $(\mathrm{V})$, vesicles $(\mathrm{v})$ and pinocytosis arrows in panel C. Panel D demonstrates the protective quality of empagliflozin. Magnification $X \times 10,000$ panel A; $\times 6000$ panel B. $\times 6000$; bar $=500$ nm panel C; $\times 4000$ panel D with scale bars $0.2 \mu \mathrm{m}, 0.5 \mu \mathrm{m}, 0.5 \mu \mathrm{m}$ in panels $\mathrm{A}, \mathrm{B}, \mathrm{D}$ respectively. Image available via license CC by 4.0 [4].

Also, the EC in the diabetic $d b / d b$ model demonstrated the following remodeling changes: $i$. endothelial thinning and loss of electron density; ii. increased vesicles-pinocytosis-vacuoles; iii. aberrant mitochondria; $i v$. hyperplasia with some reduplication; $v$. endothelial cell activation with adherent erythrocytes and leukocytes (lymphocytes and monocytes). Importantly, the EC is capable of synthesizing at least 2 main autocrine paracrine vasodilating molecules nitric oxide (NO) and prostaglandin E2 (PGE2) or prostacyclin and the autocrine paracrine potent vasoconstrictor endothelin-1 (ET-1). Recently, normal aging in Wistar rats increases EC permeability in 14-16month-old cohorts as compared to younger cohorts at 2-3 month of age [61]. Bors L. et al were able to demonstrate that there was increased thickness of EC BM, TJ/AJ were attenuated, ACfp were extended with increased GFAP staining and a decrease in the efflux permeability glycoprotein (P-gp) or the ABCB-1 efflux EC protein [61]. These findings correlate with previous sections 1.1 and 1.2. The endothelium is responsible for synthesizing the luminal ecGCx and also the inner BM that are continuous with the Pc assisted synthesis and maintenance of the inner and outer BMs of the NVU.

\subsubsection{Endothelial Glycocalyx (ecGCx)}

The ecGCx is a decorative coat of many colors and consists of a sugar-protein endothelial screenlike mesh; gel-like slime surface coating that covers and decorates the luminal side of the highly polarized endothelium and is synthesized primarily by the EC with some plasma contributions of albumin, fibrinogen and soluble plasma proteoglycans and glycolipids (Fig. 11). 


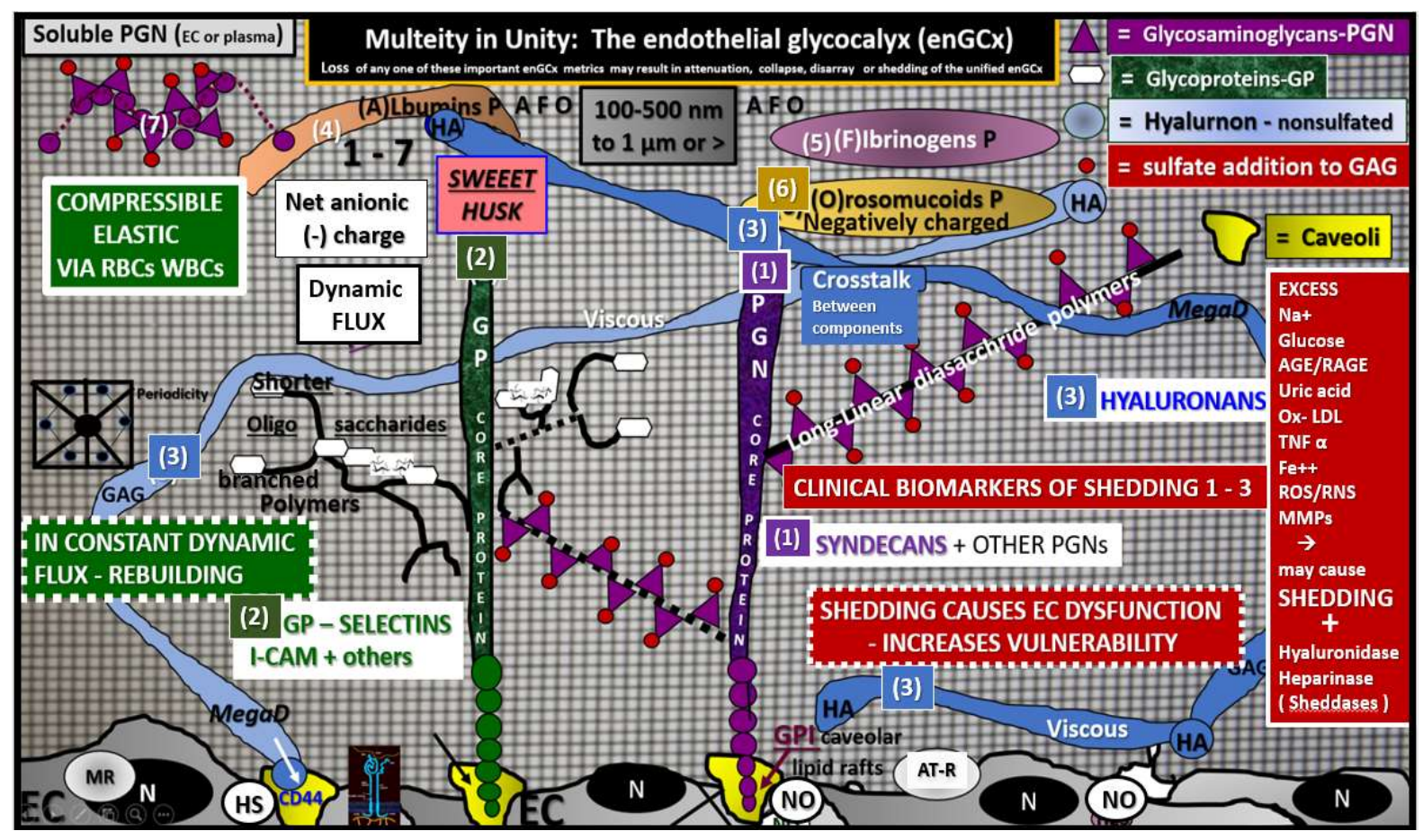

Figure 11. The endothelial cell glycocalyx (ecGCx) of the capillary neurovascular unit. This cartoon depicts the three major anchoring proteins of the ecGCx: proteoglycan(s) (PGN) (1) (purple), glycoproteins (GP) (2) (green) and Hyaluronan (HA) (3) (blue). This ecGCx sweet husk of the endothelial cell (EC) is in a constant state of flux being constantly resynthesized by the EC and is highly compressible by the blood's cellular constituents (red and white blood cells RBC, WBC). The proteoglycans (PGN) are noted for their syndecans (SYN) (plus others) and the glycoproteins (GP) are known for their selectin's such as I-CAM, P and E-Selectin plus others. One will note the glycosaminoglycans (GAGs) long-linear disaccharides of the PGNs and shorter highly branched GAGs oligosaccharides of the glycoproteins. This surface layer has a net negative anionic charge (due to orosomucoids protein and opposes negatively charged molecules in the blood utilizing Van der Wall forces) and size selective semipermeable surface barrier (hindering access of 70 kiloDalton$\mathrm{kDa}$ or larger proteins) and additionally serves as a glucose and sodium sink initially in health that can be rapidly overcome in diabetes and clinical excesses of sodium chloride salinity. The intact ecGCx contains the protective extracellular superoxide dismutase (EC-SOD) and antithrombin III (AT III) while serving as a mechanotransducer. The thickness of the ecGCx varies from 100-500nm (300nm mean), from 1- 2 micrometer in length and the percentage of EC covered by the ecGCx was approximately $40 \%$ in the NVU capillaries in the transmission electron microscopic studies and was greater in the brain as compared to the heart or lung continuous capillaries without a BBB (Fig. 9) [67]. Glucotoxicity; oxidative stress and neuroinflammation (as in the diabetic $d b / d b$ model); traumatic shock injuries; ischemia/reperfusion and sepsis are known to be capable of ecGCx shedding and/or reduction in volume and plasma levels of syndecans, I-CAMs, selectins and hyaluronans can be measured clinically with elevated levels reflecting the reduced ecGCx due to thinning, loss or shedding. $A T-1 R=$ angiotensin 1 receptor; $B B B=$ blood-brain barrier; $C D 44=$ the hyaluronan receptor of the ecGCx and cell-surface glycoprotein involved in cell-cell interactions and binds HA via the amino-terminal domain; $E C=$ endothelial cell; $G P=$ glycoprotein(s); H2S = hydrogen sulfide; HA = hyaluronan; $I-C A M=$ intercellular adhesion molecule $1 ; M R=$ mineralocorticoid receptor; $N=$ nucleus; $P=$ protein; $P G N=$ proteoglycans; $R B C=$ red blood cell; $W B C=$ white blood cell.

The intact ecGCx is important for vascular wall arteries, arterioles and capillary integrity and it does not decorate veins or venules [62]. The ecGCx is anchored to the EC via proteoglycans (syndecans plus others) and glycoproteins (selectins plus others including I-CAM), while the third component protein consists of hyaluronan. Hyaluronans may be free or anchored to the endothelium via CD44. Interestingly, this protective coating is very similar to the brain's interstitial extracellular 
matrix in molecular contents, which is difficult to examine by ultrastructure imaging due to its extreme thinness even at very high magnification due to the tightly packed glia and neurons within a confined space created by the exoskeleton the skull. The elusive ecGCx is in a constant state of flux and also in a constant state of being regenerated and repaired by the endothelium and plasma constituents $[63,64,65,66]$. The systemic cells and molecules of the blood seldom see or come into direct contact with the plasma membrane-plasmalemma of the EC, because it is constantly and nearly continuously covered by the protective ecGCx surface coating, which prevents direct exposure of systemic blood components to the EC plasma membrane in health.

Recently, the brain's NVU ecGCx has been beautifully studied, visualized and documented by lanthanum nitrate staining by both transmission electron microcopy (TEM) and scanning electron microscopy (SEM) making this EC surface coating layer visible even though TEM preparations undergo numerous dehydration steps [67]. The perfusion fixation with lanthanum nitrate presacrifice allows the ecGCx to be beautifully stained and visualized at the time of TEM and SEM studies from cortical brain regions of the C57B6 mice (Fig. 12).

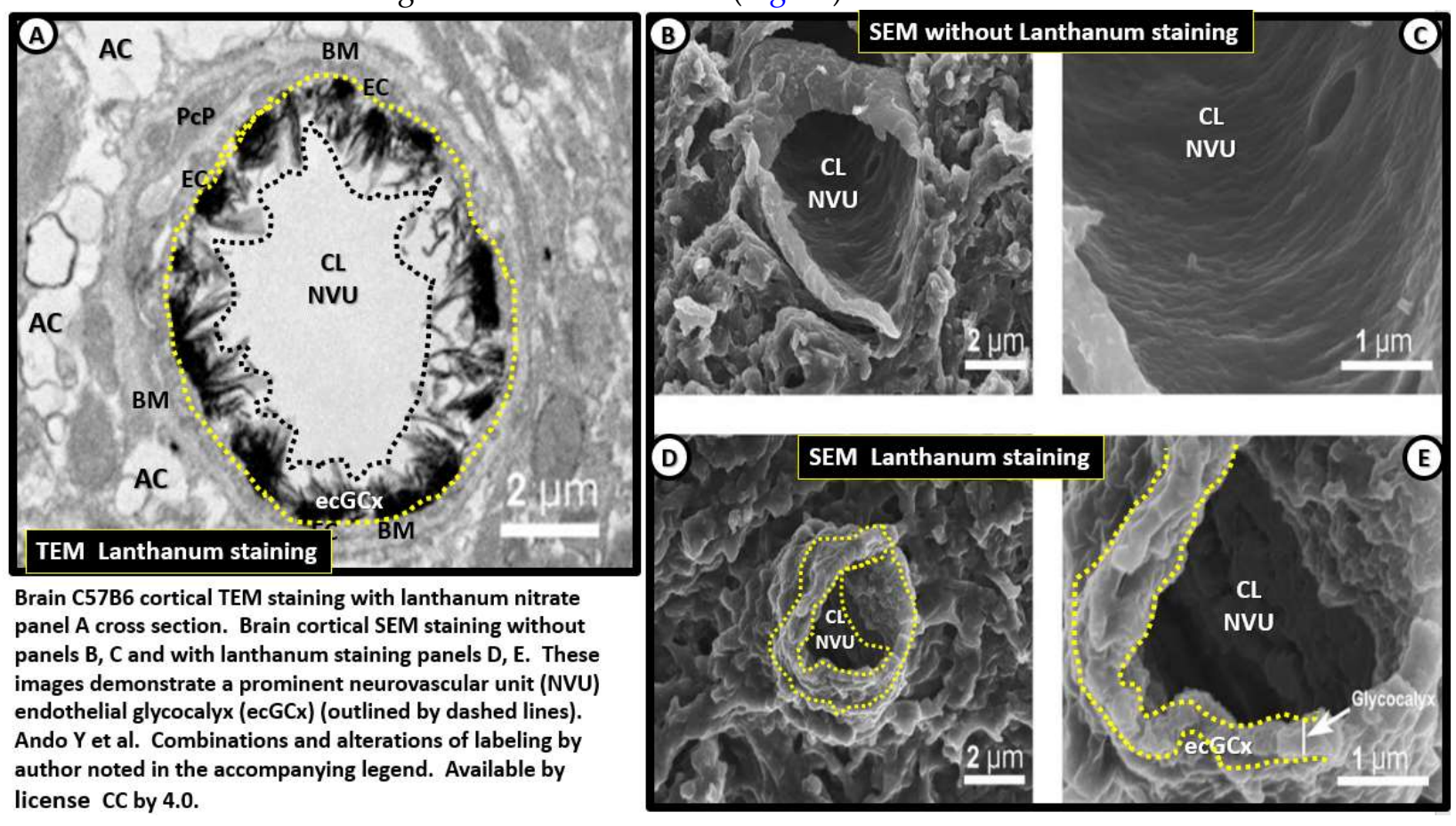

Figure 12. TEM and scanning electron micrographs of the endothelial cell glycocalyx. With And Without Lanthanum Staining. Panel A depicts a transmission electron microscopic (TEM) cross sectional image of the endothelial cell glycocalyx (ecGCx) of the neurovascular unit, outlined by yellow dashed lines abluminally and black dashed line luminally. Note how this electron dense stained ecGCx by lanthanum nitrate with perfusion fixation covers nearly the entire luminal surface of the endothelial cell (EC) with intermittent varying electron dense cores and extend outwardly into the lumen in control non-diseased models of the C57B6 mice from the cerebral cortical brain. Scale bar $=2 \mu \mathrm{m}$. Panels B and C illustrate scanning electron microscopy (SEM) micrographs the NVU without lanthanum nitrate staining and panels D and E depict lanthanum nitrate staining and now, note the visible elusive ecGCx outlined by yellow dashed lines from the EC on the luminal side. This image actually combines figures 1 and figures 2 from Ando Y [67]. Scale bar $=2 \mu \mathrm{m}$ Panels A, B and D. Scale bar $=1 \mu \mathrm{m}$ panels C, E. Image available via license CC by 4.0 [67].

Recently, our transmission electron microscopy core was surprisingly able to identify a highly electron dense endothelial surface coat utilizing a secondary staining technique that is utilized for focused ion beam/scanning electron microscopy (FIB/SEM) with the use of traditional electron microscopy (osmium tetroxide, potassium ferrocyanide, en bloc uranyl acetate and Walton's Lead Nitrate solution staining) in control C57B6 mouse models (Fig. 13). 


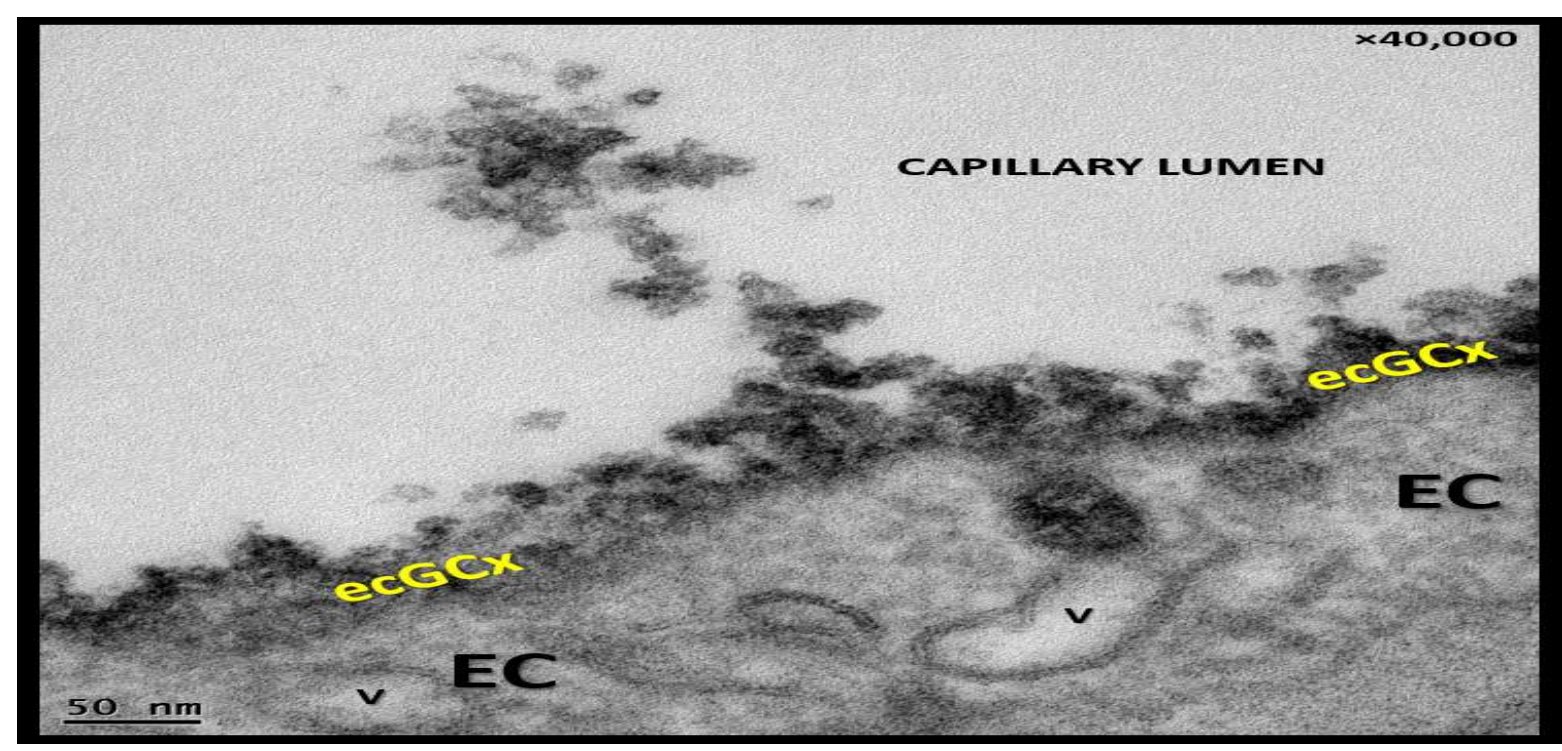

Figure 13. Transmission electron microscopy identification of endothelial cell glycocalyx. Our TEM core was recently able to identify the extremely electron dense endothelial glycocalyx (ecGCx). Note base regions with approximately 50nm thickness with periodic spikes in thickness of 200-300nm on the surface capillary luminal side of the endothelial cell (EC). Also note within EC cytoplasm the presence of large $50-100 \mathrm{~nm}$ vacuoles $(\mathrm{V})$. Magnification $\times 40,000$; scale bar $=50 \mathrm{~nm}$. This is an original image not previously published.

Also, our core has more recently been able to identify the elusive ecGCX with perfusion fixation by lanthanum nitrate in the cerebral NVU capillaries of control C57B6 mouse models and it will be interesting to observe for shedding, degradation and/or thinning of this endothelial surface coating in age-matched diabetic models in the coming future (Fig. 14).

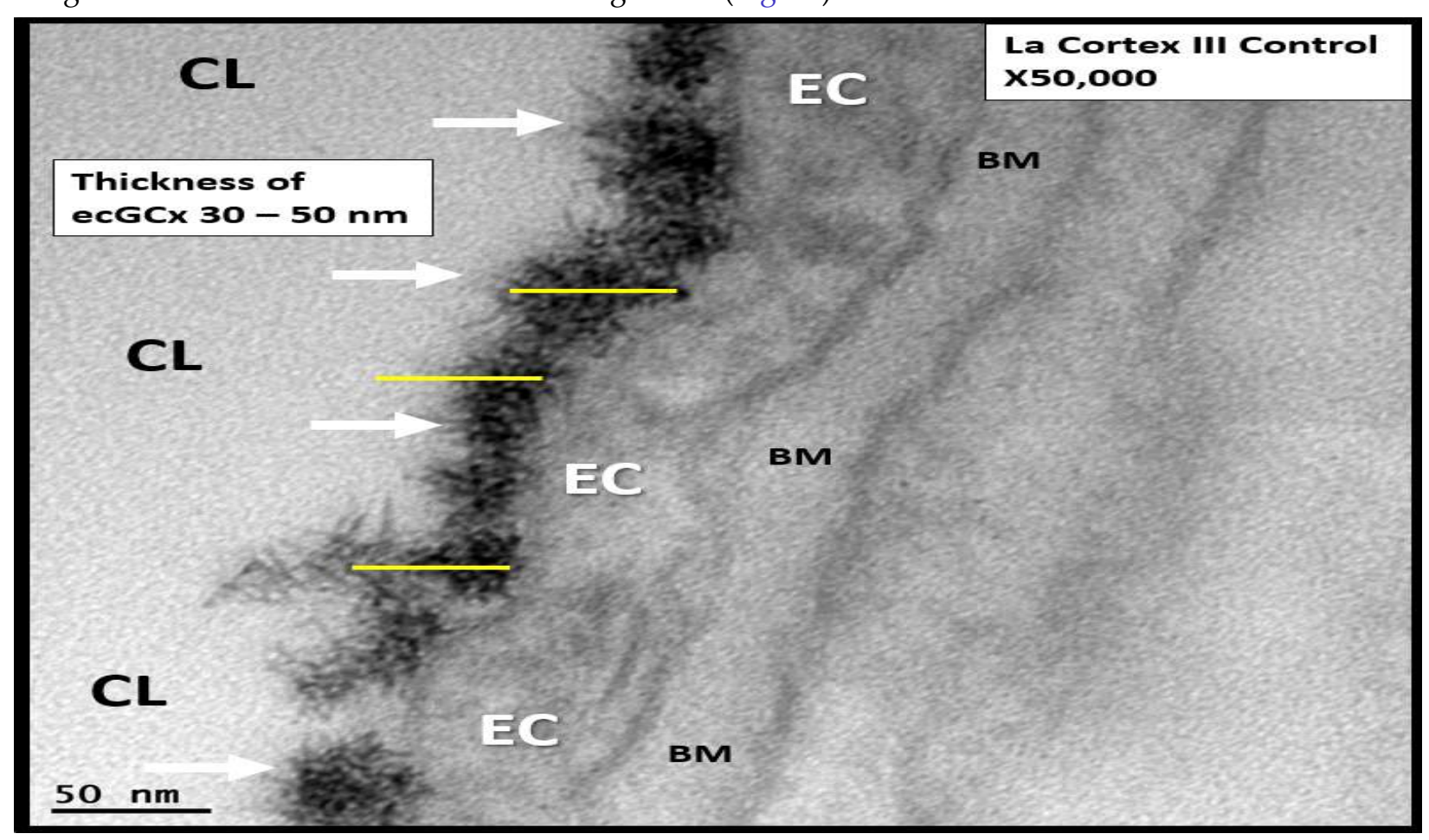

Figure 14. Lanthanum nitrate perfusion fixation staining of control C57B6 mouse models in the cortical layer III capillary neurovascular unit endothelial cell glycocalyx (ecGCx). Note the extreme electron dense staining of lanthanum nitrate covering the endothelial surface of the ecGCx (arrows) similar to figures 12 and 13 that notably does not penetrate the endothelium or any other aspects of the neurovascular unit with lanthanum nitrate staining. Its thickness appears to vary from 30 to 50 
nanometers $(\mathrm{nm})$. Yellow scale bars of $50 \mathrm{~nm}$ are placed overlying the electron dense lanthanum nitrate staining ecGCx. Magnification x50,000; scale bar $=50 \mathrm{~nm} . B M=$ endothelial cell basement membrane; $C L=$ capillary lumen; $E C=$ endothelial cell cytoplasm.

Aging is known to be the major risk factor for the development of LOAD, and it has recently been demonstrated that aging results in a thinning of the ecGCx by $50 \%$ when comparing young (6 months) to old (24 months) C57B6 mice (mesenteric and skeletal microvessels) and 30\% thinning of the ecGCx when comparing young ( 23 years) and old ( $60 \pm 2$ years) humans (sublingual microvessels) [68]. Now that we may have a reliable and reproducible method for determining ecGCx disruption, thinning, degradation and/or shedding we may be able to demonstrate by TEM with perfusion fixation staining of lanthanum nitrate that indeed there is disruption of the endothelial surface layer - ecGCx and in some cases be able to determine if the increased permeability that was once thought to be non-disruptive may now be considered to be disruptive due to alterations of the ecGCx [69].

\subsubsection{Endothelial cell Basement Membrane (BM)}

The BM consists of type IV collagen, fibronectin, hyaluronan, laminin, nidogen, and heparin sulfate proteoglycans (agrin and perlecan) and envelops the basilar portion of the EC and splits to encompass the Pc and forms its base to which the EC and Pc BM forms the adhesion anchoring structure for the corona of ACfps [4, 70]. The BM is synthesized primarily by the EC; however, the Pc also contributes to the synthesis and maintenance of the NVU BM. Interestingly, as one studies the ultrastructure of the NVU, one can note that the polarized ECs create a barrier at the luminal surface layer (ecGCx) and also at the basilar abluminal side of the EC's monolayer, the BM. In the obese insulin resistant type 2 diabetic $d b / d b$ model there is BM thickening and even though it is thickened, its structure is remodeled with rearrangement and thickening, which allows for increased permeability (Fig. 15, 16).

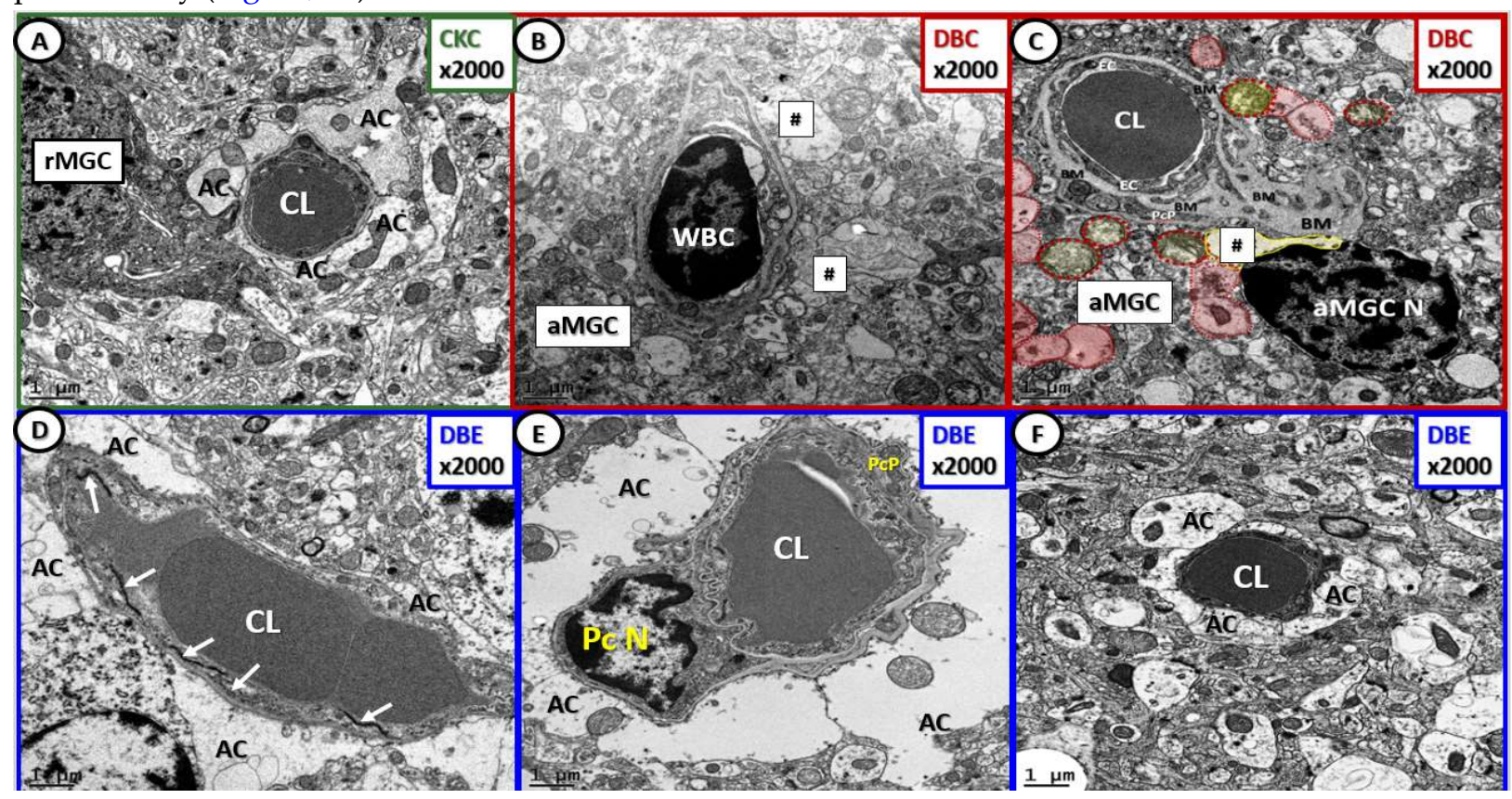

Figure 15. Empagliflozin protects the neurovascular unit from basement membrane thickening: Loss Of blood-brain barrier endothelial tight and adherens junctions and pericytes. Panel A and D depict the normal ultrastructure of the basement membrane (BM) and BBB in control non-diabetic CKC models. Panels B and C depict the Pc ghost (panel B) and complete loss of Pcs in panels B and C as well as the detachment of astrocyte foot processes in panel A (hashtags) with invading activated microglia cell (aMGC) in the $d b / d b$ DBC diabetic models outlined in red. Panels D, E and F demonstrate that empagliflozin protects from EC activation, loss of EC tight and adherent junctions (arrows), Pc loss and AC detachment in the DBC treated models with empagliflozin (DBE) outlined in blue. Magnification X2000;scale bar $=1 \mu \mathrm{m}$. AC = Astrocyte end feet processes; aMGC = activated 
microglial cell; $\mathrm{CL}$ = capillary lumen; $\mathrm{Pc} \mathrm{N}$ = pericyte nucleus; $\mathrm{PcP}=$ pericyte process; $\mathrm{rMGC}$ = ramified microglia cell. Image available via license CC by $4.0[4,7]$.
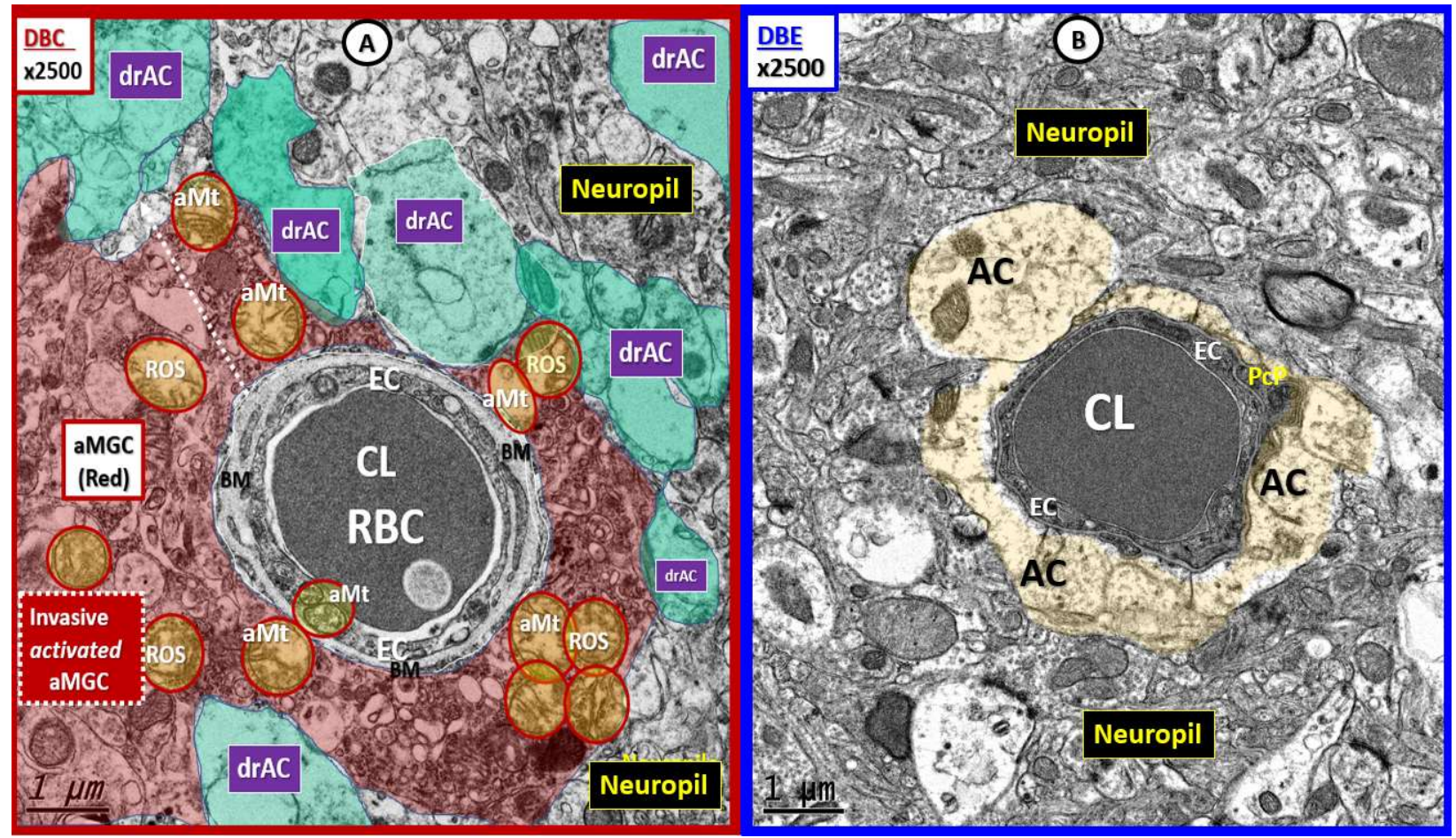

Figure 16. Basement membrane thickening, activated microglia invasion and detachment of astrocyte foot processes are protected with empagliflozin. Panel A depicts the invasion of the activated MGC (aMGC) to totally engulf the NVU with detachment of AC end foot processes (pseudo-colored green; $\mathrm{drAC}=$ detached retracted astrocyte). Also, note the aberrant mitochondria (aMt) (pseudo-colored yellow with red outline) in DBC responsible for reactive oxygen species (ROS) production and leakage. Panel B illustrates how empagliflozin protects the NVU from aberrant remodeling in DBC treated with empagliflozin (DBE) outlined in blue. Note the intact AC end feet (pseudo-colored golden) and that the mitochondria in DBE are electron dense and not aberrant as in the DBC. Panel B is a higher magnification of panel $\mathrm{F}$ in figure 3. Magnification $\mathrm{x} 2500$; scale bar $=1 \mu \mathrm{m}$. $a M G C=$ activated microglia cell; $a M t=$ aberrant mitochondria; $d r A C=$ detached retracted $A C E C=$ endothelial cell; $C L$ $=N V U$ capillary lumen; $P_{c}=$ pericyte foot process; $R B C=$ red blood cell; $R O S=$ reactive oxygen species. Image available via license CC by 4.0 . $[4,5,7]$.

\subsubsection{Neurovascular Unit (NVU) Pericyte(s) (Pc)}

The pericyte $(\mathrm{Pc})$ is a ubiquitous - systemic (found both in peripheral and brain microvascular capillaries), requisite, mesenchymal derived, pluripotent and postnatally undifferentiated vascular mural cell important for mediating physiological and pathological repair processes. The Pc serves other microcirculation functions including post-natal vascular development (angiogenesis), important for maturation and remodeling of the NVU. Additionally, pericytes provide microvascular structural stabilization as well as provide a supportive-protective role to capillary endothelial cells and is known to be an innate immune and antigen presenting cell of the NVU and may be capable of differentiating into a microvascular niche mesenchymal stem cell (Fig.17, 18) [71, 72, 73, 74, 75, 76, 77, $78,79,80]$. 


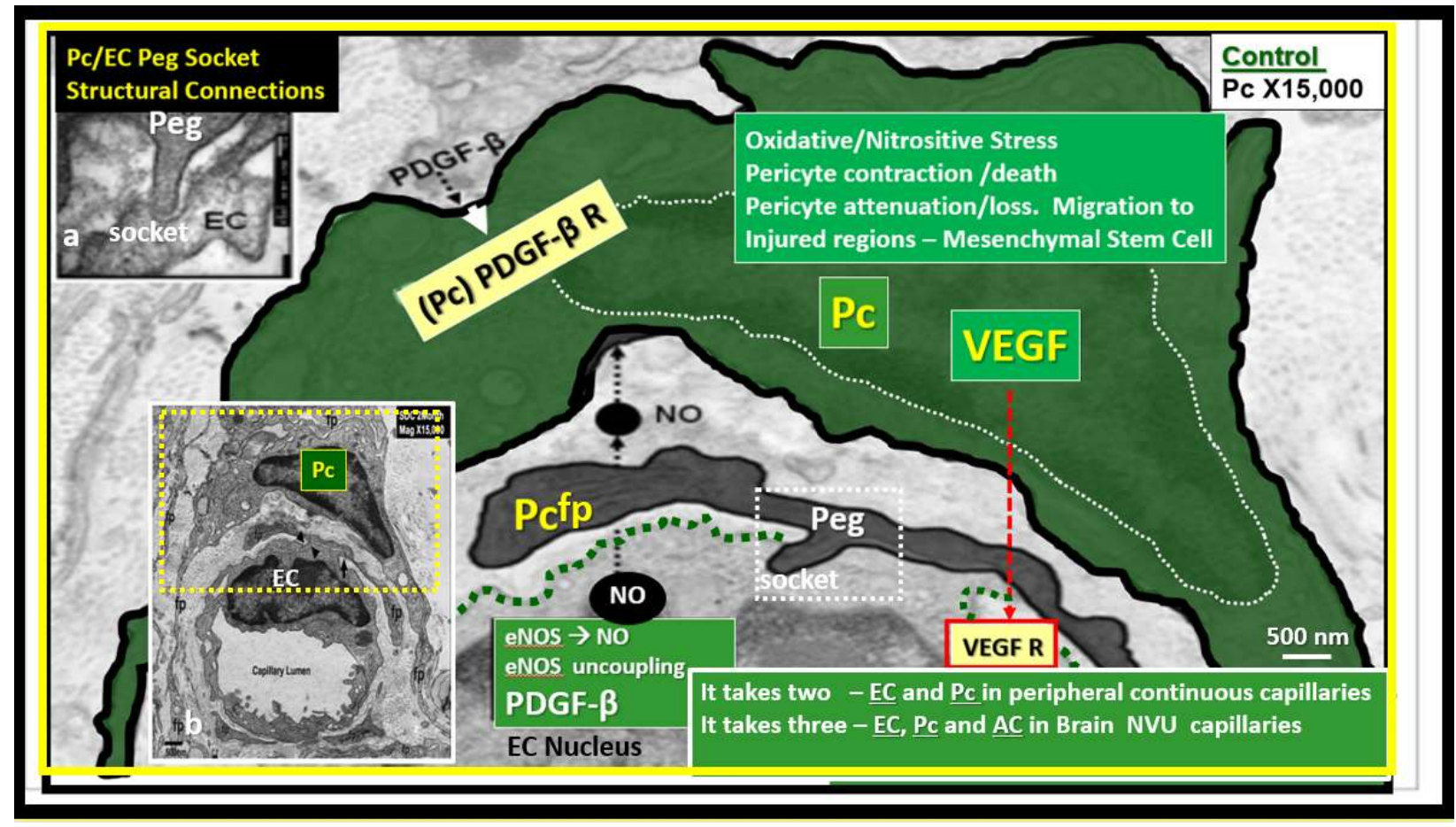

Figure 17. Normal pericyte - endothelial morphology and crosstalk in control CKC models. In peripheral microvessels such as the continuous capillaries "It takes two". Indeed for both peripheral continuous capillaries and brain continuous capillaries of the neurovascular unit (NVU with bloodbrain barrier (BBB) tight and adherens junction (TJ/AJ)) it takes these two highly integrated vascular mural cells (endothelial cells (EC) and pericyte (Pc) interaction and crosstalk. However, in the brain "it takes three" since ECs, PCs and astrocytes (ACs) must properly interact in order for the proper development and maintenance of the BBB TJ/AJ - junctional adherens and zona-occludin-1 (ZO-1) as well as the glial astrocyte $(\mathrm{AC})$ to allow for this microvascular system to develop proper cerebral blood flow, prevent increased permeability, and assure proper NVU coupling with regional neurons to assure proper functional hyperemia. This image focuses on peripheral continuous capillaries in order to demonstrate normal EC-Pc morphology. The Pericyte soma is pseudo-colored green and its nucleus is enveloped with white dashed line. Importantly the Pc stains positive for alpha smooth muscle actin and allows for contraction and importantly relaxation to allow for NVU coupling and vasodilation. Insert (a) depicts the peg socket interaction while insert (b) allows the entire capillary to be viewed in its environment without colorization. Magnification $\times 15,000$; bar $=500 \mathrm{~nm}$ in all images. Also, note that the EC provides nitric oxide (NO) and platelet derived growth factor (PDGF $\beta$ ) and that the Pc provides vascular endothelial growth factor (VEGF) and makes possible the contraction and relaxation of the capillary NVU via Pc actin which is essential for these two cells survival via crosstalk for proper function and protection of the endothelial capillary NVU of control CKC models. eNOS = endothelial nitric oxide; NVU = neurovascular coupling; $P D G F-\beta=$ platelet derived growth factor beta; $P D G F-R$ its receptor; VEGF = vascular derived growth factor. and VEGF receptors - VEGF-2 (Flk-1/KDR = type III receptor tyrosine kinase).

Changes in Pc biology are implicated in a variety of microvascular alterations, including wound healing, diabetes, hypertension, inflammation, neoplasia, vascular calcification [80] greatly involved in T2DM, aging and LOAD (Fig. 18). 


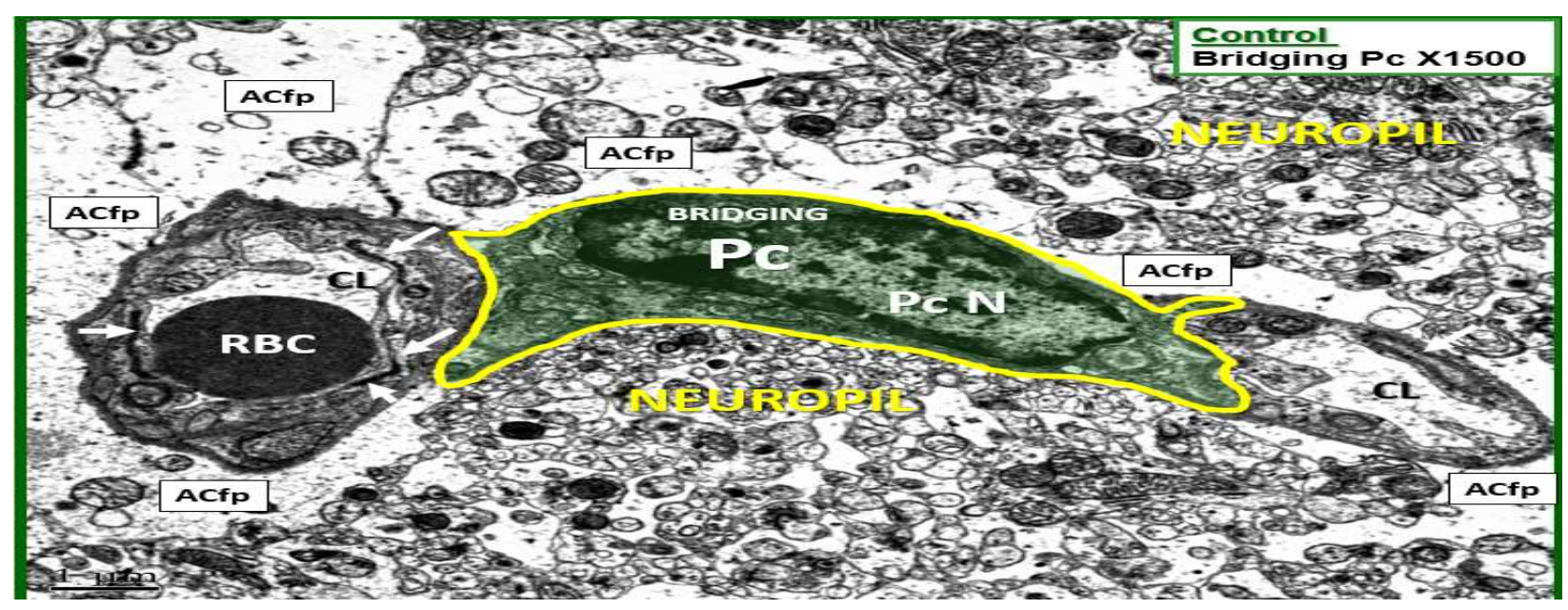

Figure 18. Pericyte connectivity between two capillary neurovascular units in cerebral cortex of the C57B6 mouse control model layer III. ACfp = astrocyte foot processes; arrows = tight and adherens junctions; $C L=$ capillary lumen; $P c=$ pericyte; $P c N=$ pericyte nucleus.

Importantly, pericytes are contractile cells and contribute to the regulation of capillary cerebral blood flow, hydrostatic balance and maintenance of proper intracapillary pressure and permeability between the microvascular NVU and interstitial tissue [73, 74, 75, 76, 77,78, 79]. The EC provides the Pc with essential NO and platelet derived growth factor beta (PDGF $\beta$ ) to allow for prevention of contraction and allows for dilation of the capillary NVU - capillary neurovascular unit maintenance. Also, the EC relies on the Pc to provide VEGF production (essential cellular crosstalk). While each of these above interactions between pericytes and endothelial cells are extremely important it is beyond the scope of this review to discuss them in their entirety, especially in wound healing and angiogenesis.

Previously, some have considered the Pc to be the "guardian angel" or sentinel of the EC microcirculation within the peripheral continuous capillaries (Fig. 17, 18) [61, 80, 81, 82, 83]. This same "guardian angel" analogy may also be applied to the EC of capillary NVU as a functional and structural unit in the brain, since platelet derived growth factor beta receptor (PDGFR-B) knockout models in Pcs have demonstrated the abnormal formation of the NVU in adult models with increased permeability due to impaired BBB TJ/AJ development and function and impaired clearance of A $\beta$ $[73,74,75,76,77,78,79]$. Additionally, it's very important role within the NVU has undergone considerable expansion regarding its being the cell responsible for the effect of the regional neuronal activity of the neurons via the connecting astrocyte to result effectively in dilation in order for NVU coupling and functional hyperemia to support the necessary vasodilating properties of the capillary NVU and the smallest of arterioles [4, 7, 73, 74, 75, 76, 77, 78, 79].

Recently, it has been shared that pericytes (Pc) in the female diabetic $d b / d b$ model undergoes marked remodeling changes including attenuation, retraction and/or complete loss from the cortical layer III NVU. These remodeling changes have also been demonstrated to be protected with empagliflozin treatment for 10 weeks duration from 10 to 20 weeks of age (Fig. 19) [4, 7]. 


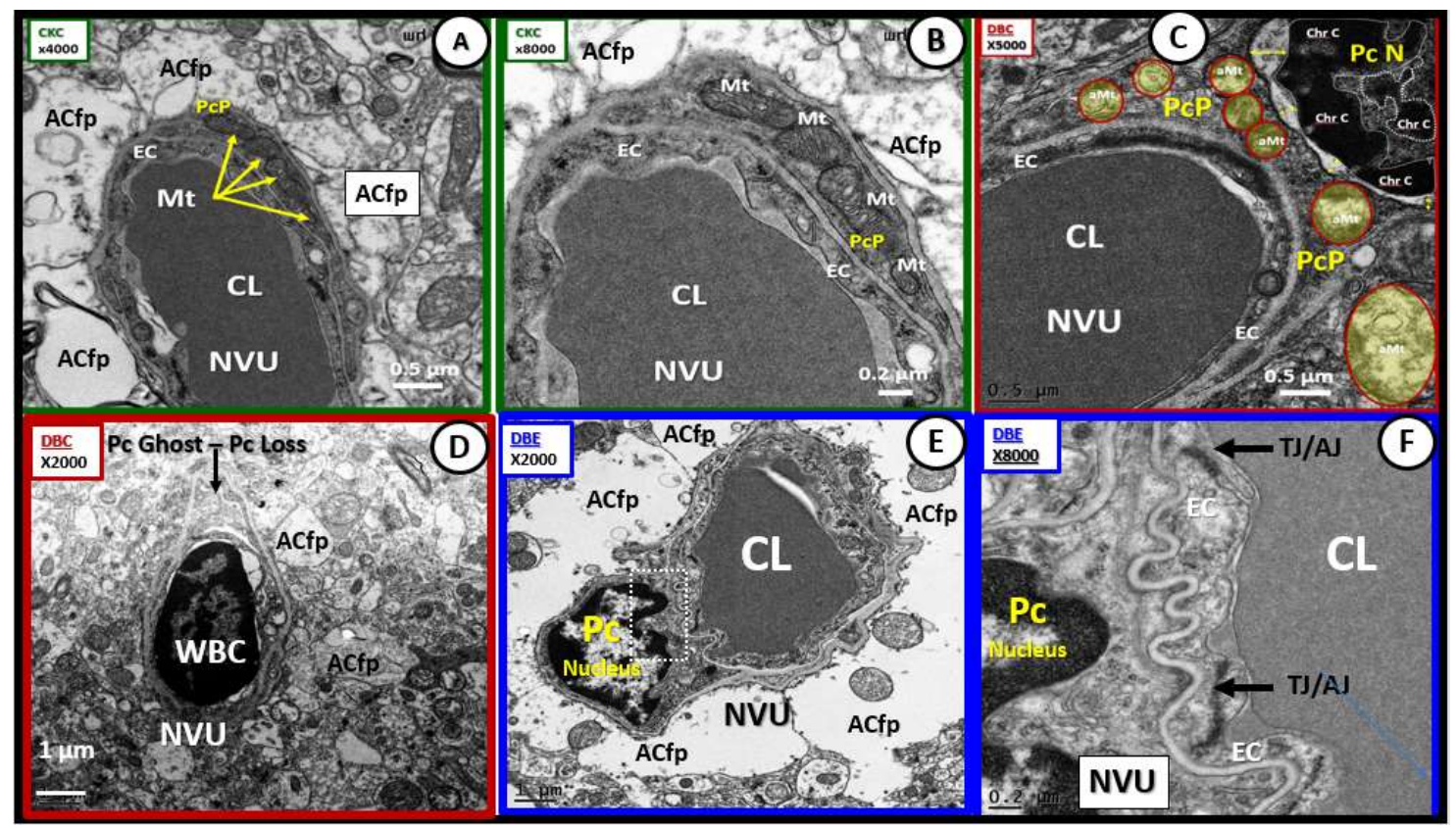

Figure 19. Pericyte injury and loss in diabetic female $d b / d b$ with protection by empagliflozin. Panels $\mathrm{A}$ and $\mathrm{B}$ demonstrate the normal appearance of pericyte (Pc) and foot processes (Pcfp) in relation to the neurovascular unit (NVU) endothelial cell (EC) in control CKC models. Magnification $\times 4000$; bar $=0.5 \mu \mathrm{m}$ in $\mathrm{A}$ and $\times 8000$; bar $=0.2 \mu \mathrm{m}$ in $\mathrm{B}$. Panel $\mathrm{C}$ illustrates injury - abnormalities (aberrant mitochondria and Pc Nuclear retraction and increased chromatin condensation) in diabetic DBC outlined in red. Magnification $\times 3000$; scale bar $=0.5 \mu \mathrm{m}$. Panel D illustrates an activated endothelial cell with white blood cell adherence (lymphocyte) with loss of pericyte and foot processes in diabetic DBC outlined in red. Magnification $\times 1000$; bar $=0.1 \mu \mathrm{m}$ ghost cell. Panels E and F depict the protection of the Pc and Pcfp in the empagliflozin treated modes (DBE). Magnification $\times 2000$; bar $=1$ $\mu \mathrm{m}$ (panel E) and magnification $\times 8000$; bar $=0.2 \mu \mathrm{m}$ (panel F). Image available via license CC by 4.0 [4].

\subsubsection{Astrocyte (AC)}

ACs are unique cells localized to only the brain and spinal cord tissues and they are the major strategic connecting cell within the brain. The astrocytic end-feet - foot processes (ACfp) of the capillary NVU are tightly adherent to the BM of the EC and Pc cells and play a specialized role for water, amino acid and ionic homeostasis; provide a reservoir for glucose storage via glycogen and are the primary source within the brain for localized antioxidant production of glutathione in addition to communicating with adjacent $\mathrm{AC}$ and bidirectionally with regional neurons for functional hyperemia - NVU coupling and cerebral blood flow (Fig. 7, 12, 15, 16, 18, 19) [4, 84, 85, 86, 87, 88, 89, 90]. Importantly, AC foot processes (ACfp) connect regional neurons to the mural cells of the NVU and are responsible for signaling the mural cells via glutamate signals, increases in calcium transients (Ca++ flux) from the neurons to the mural cell pericyte to dilate in the presence of increased neuronal activity and create the capillary NVU coupling which results in functional hyperemia and CBF. $\mathrm{ACs}$ are also connective in that they form a syncytium amongst other $\mathrm{AC}$, which are in a constant state of sending or receiving information from other like AC cells. While it is a well-known and accepted theory that active neurons increase their nutrient-energy supply and oxygen by dilating nearby arterioles and capillary NVUs to provide for neurovascular coupling or functional hyperemia there does remain somewhat of controversy regarding whether it is the arteriole or capillary that play the major role. It is the opinion of the author that even though the capillary NVU may be the first to dilate that both capillary and arterioles of the brains vascular supply are affected. To this end, Mishra A et.al. have recently demonstrated that $\mathrm{AC}^{\prime}$ s signal pericytes but not the VSMCs of arterioles [91].

It is important to note that in the obese, insulin resistant and T2DM $d b / d b$ models that the AC end-feet of the capillary NVU were detached, separated and retracted (Fig. 15B,C; 16A; 19D) [92]. 
Importantly, this would result in the loss of the connecting ACs from regional neurons to the capillary NVU and impair functional hyperemia (Fig. 20, 21, 22) [4, 7, 92]. These ultrastructural changes in the preclinical rodent $d b / d b$ models if found to be present in human brain tissues would impair functional hyperemia and result in decreased CBF and could contribute to a loss of energy substrate sources and decrease in oxygen resulting in ischemia and premature neuronal dysfunction in synapses and eventually dysfunction with known cognitive impairment and neurodegeneration. This detachment may be due to the increased endothelial, pericyte and AC oxidative stress generating excessive ROS/RNS due to glucotoxicity as previously proposed [4, 7, 91, 92, 93], which was protected with a SGLT2 inhibitor (empagliflozin) (Fig. 20) [7].

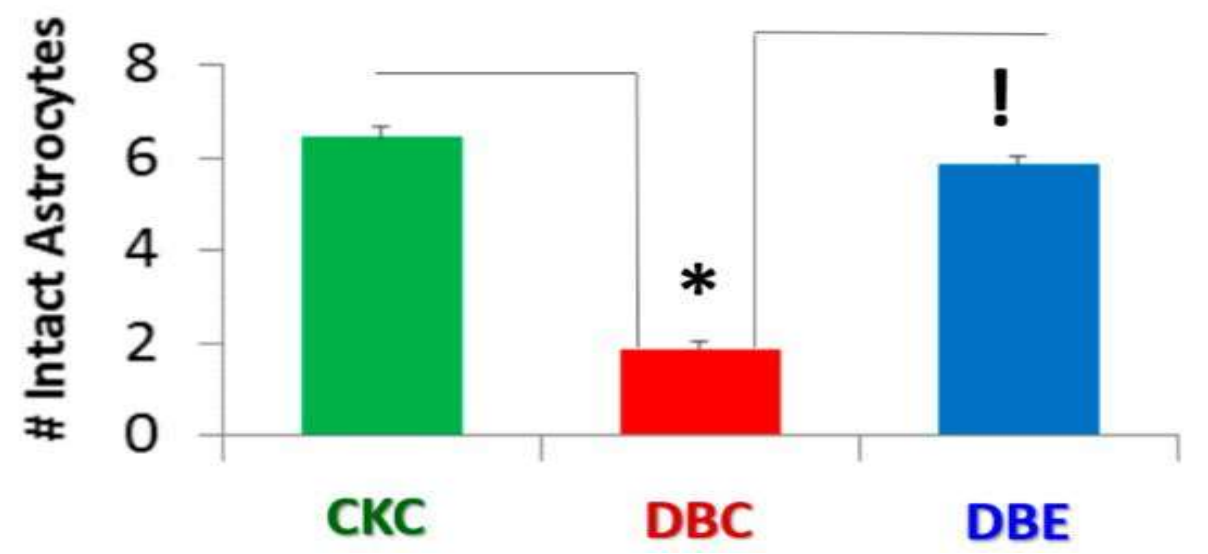

Figure 20. diabetic $d b / d b$ models have a significant decrease in the number of intact and attached astrocyte foot processes (ACfp) at the outer basement membrane of the capillary neurovascular unit (NVU). This image represents the mean number (\#) of astrocytes (ACfp)/NVU that are firmly attached and abut to the outer endothelial and pericyte outer basement membranes of the capillary neurovascular unit (NVU), numbering 22 in CKC (control heterozygous: green), 25 in DBC (diabetic $d b / d b$ : red), and 22 in $d b / d b$ empagliflozin-treated (DBE: blue) for a total of 69 capillary NVUs counted. The mean number of ACfp/NVU was $6.4 \pm 1.1 \mathrm{in} \mathrm{CKC,} 1.88 \pm 0.72$ in diabetic DBC, and 5.86 \pm 0.88 in diabetic $d b / d b$ treated with the SGLT2 inhibitor (empagliflozin) DBE. ${ }^{*}$ CKC compared to DBC ( $p$-value < 0.05); ! DBC compared to DBE ( $p$-value < 0.05). Image available via license CC by 4.0 [92].

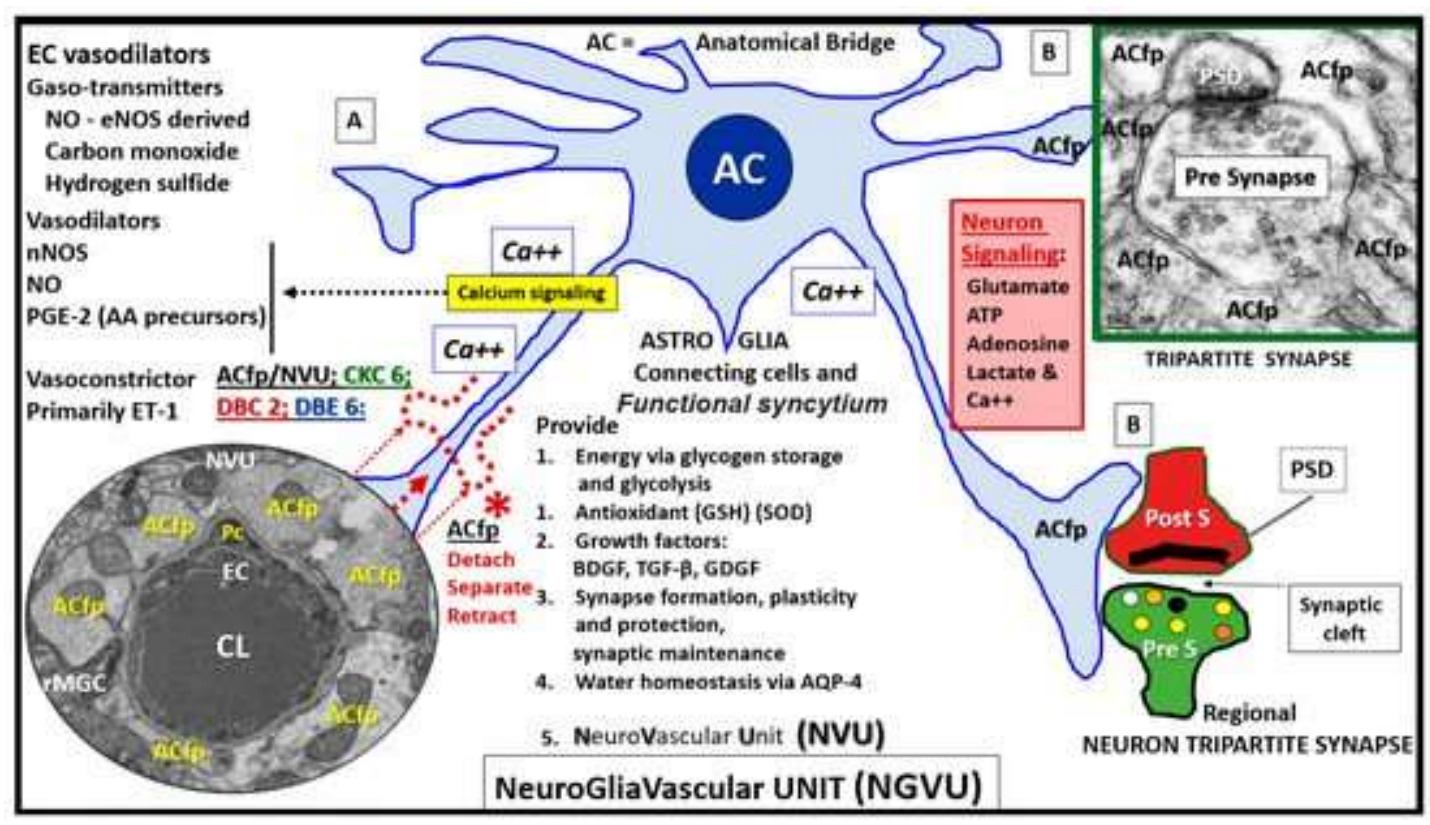

Figure 21. astrocyte connectivity between the capillary and neurons to form the neurovascular unit. This image illustrates the combination of cartoon and transmission electron micrographs (TEM). 
(A and B) The TEM image of the neurovascular unit (NVU) with magnification $x 4000$ and the TEM image (Figure 5B); magnification $\times 20,000$; scale bar $=100 \mathrm{~nm}$. Image available via license CC BY 4.0. [92].

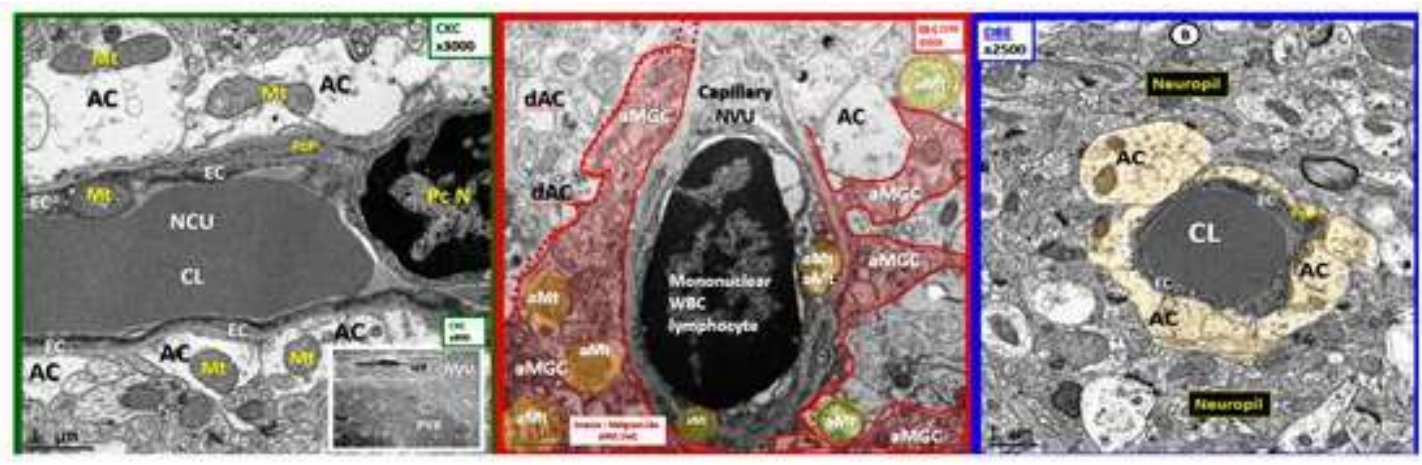

Figure 22. prevented astrocyte (AC) detachment from the neurovascular unit (NVU). Left panel is control (CKC) and note the corona of electron lucent AC foot processes enveloping the NVU. The mid-panel diabetic $d b / d b$ model depicts an invasive activated microglia cell (aMGC) that is associated with marked AC detachment. The far right illustrates that treatment with empagliflozin protected the NVU AC from being injury, detachment, separation and retraction. Scale bar $=1 \mu \mathrm{m}$ in each image with various small differences in magnification at top. Image available via license CC BY 4.0 [4,5,7].

\subsubsection{Microglial Cell(s) (MGC)}

MGCs are currently thought to be yolk sac-derived (mesoderm/mesenchymal), colonize the brain prenatally (embryonic day 10-14 in the mouse and week 4-24 in human brain), the innate first line of resident immune cell defense and thus, the immune guardians or gatekeepers of the brain [94, 95]. MGCs may be considered the resident immunocompentent and phagocytic cell of the CNS and are critical to its normal functioning in health and homeostasis. Interestingly, they precede the appearance of the NVU, astrogliogenesis, oligodendrogenesis, neurogenesis, migration, and myelination and are known to be regionally distributed. Ramified MGC (rMGC) are constantly at work as a consummate gardener to provide a cleaning - housekeeping function and provide surveillance for waste clean-up from normal wear and tear of the brain's cellular milieu. Their ramified processes are constantly moving about the brain ready to identify and phagocytose any unwanted accumulation of structural byproducts of metabolism in homeostasis or in response to injury mechanism $[5,96]$. In focused ion beam/scanning electron microscopic (focused ion beam/ scanning electron microscope - FIB/SEM) videos one can even observe the rMGC cytoplasmic extensions as they appear moving about between neurons and the NVUs providing these important homeostatic functions (Fig. 23, 24) [5]. 


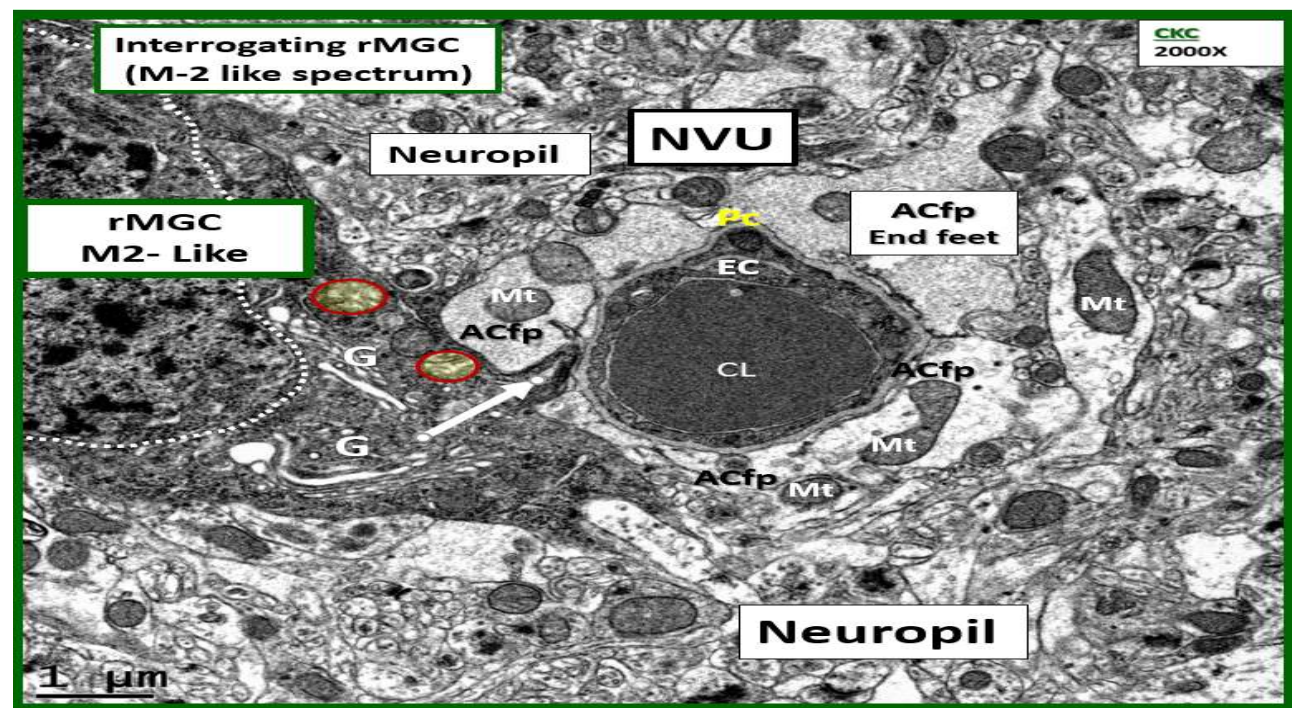

Figure 23. Ramified microglial cell (rMGC) interrogating the neurovascular unit (NVU).

This image illustrates the rMGC interrogating the intact NVU in the C57B6 control models (CKC). Note the rMGC protruding its cytoplasmic process inserting between the astrocyte foot processes (ACfp) (arrow) or end-feet ( 50-100nm space) that encompass the capillary endothelium (EC) surrounding the capillary lumen (CL). Also note the prominent Golgi system within the rMGC cytoplasm (G). Even in the rMGC there will be occasional aberrant mitochondria (pseudo-colored yellow with red outline) $(\mathrm{Mt})$. Magnification $\times 2000$; $b a r=1 \mu \mathrm{m}$. Image available via license CC BY 4.0. [4]

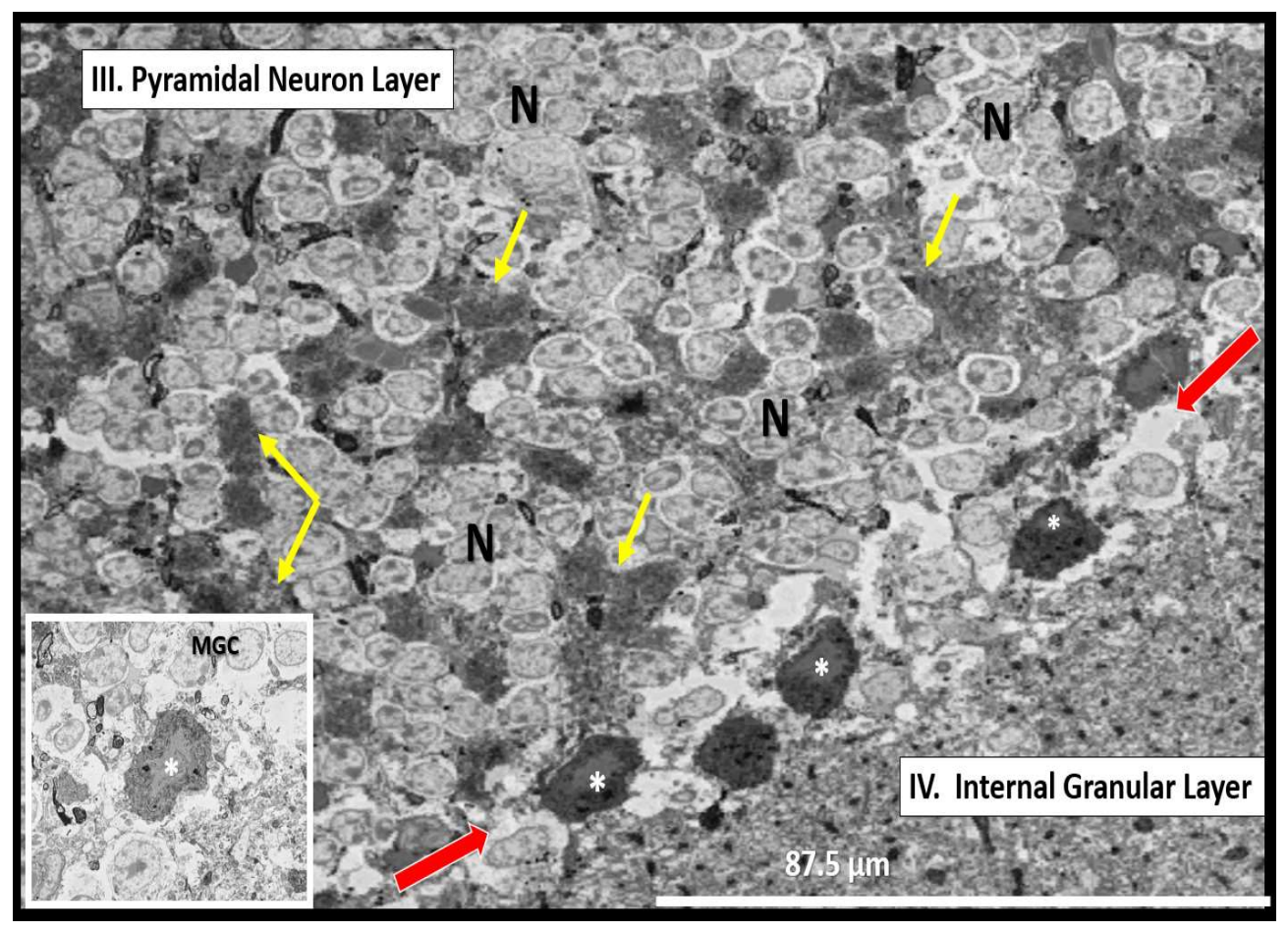

Figure 24. Ramified microglia cell(s) (rMGCs) with electron dense cytoplasmic extensions (yellow arrows) intermixed with pyramidal layer III neurons $(\mathrm{N})$ of the cortical grey matter in control CKC 16-20-week-old mice. Note what we term, a train of rMGCs. with remodeled lipid bodies to form lipid cores (asterisks) in the interstitium between layers III and IV (red arrows). Inset is an exploded highlighted image of the MGC with lipid core (asterisk). Scale bar $\sim 87.5$ micrometer. Image information from focused ion beam/scanning electron microscopy (FIB/SEM technology): Voltage: 3 kV; Pixel Size: 350 nm; Field of View: $175 \times 175 \mu \mathrm{m}^{2}$; Image Size $491 \times 497$; Vacuum: 0.25 Torr. 
MGCs have a large number of membranous/intracellular microglial markers and a large number of signaling molecules, which include numerous microglia, cytokines and chemokines [97]. Additionally, they contribute to the regulation of brain development, shaping synaptic connectivity within neuronal networks and are of major importance in brain defense injury $[5,97,98,98]$.

MGCs are readily capable of producing large amounts of free radicals (superoxide, reduced nicotinamide adenine dinucleotide phosphate oxidase (NADPH Ox), inducible nitric oxide synthase (iNOS) and mitochondrial-derived ROS and are the major killing - phagocytic cell for infectious processes in the brain. Damage/damage-associated molecular patterns (DAMPs) or pathogenassociated molecular patterns (PAMPs) and MGC remain in an activated state until the DAMPS/PAMPs signal subside. Importantly, microglia are able to return to their surveilling-ramified phenotypes once the danger-damage signals or infectious invaders have been eradicated and assume their normal cellular debris housekeeping role of rMGCs [5, 94, 95, 96, 97, 98]. While MGCs are essential to homeostasis they may also become injurious to the CNS cells as in the invasive damaging role they play in NVU BBB loss of integrity in diabetic models as a result of their invasiveness that is associated with AC detachment and retraction previously discussed in section 3.2.5. [4].

Ramified microglia cells are genetically programed to constantly be prepared to undergo a rapid diverse phenotypic remodeling functional change to what may be termed activated-amoeboid microglial cell phenotype (aMGC). These changes may be due to morphological remodeling and/or the expressions of their cell surface receptors in response to danger or damage signals such as PAMPs or DAMPs due to oxidized/glycated proteins/polypeptides, lipids, and nucleic acids from their diabetic hyperglycemic microenvironment.

Activated microglial phenotypes have been classified by some to be similar to peripheral macrophages, i.e., M1-like (classically activated macrophages) and M2-like (alternatively activated macrophages) cells $[99,100]$. However, the possibly more preferred method of identification of MGCs relies on individual cell surface markers or their response to inducible cytokines and/or neurotoxins such as LPS (lipopolysaccharide) [101, 102]. Author has chosen to utilize only the terms ramified (rMGC) or activated (aMGC) when referring to the morphofunctional-pathomorphologic phenotypic polarization in TEM observations (Fig. 25).

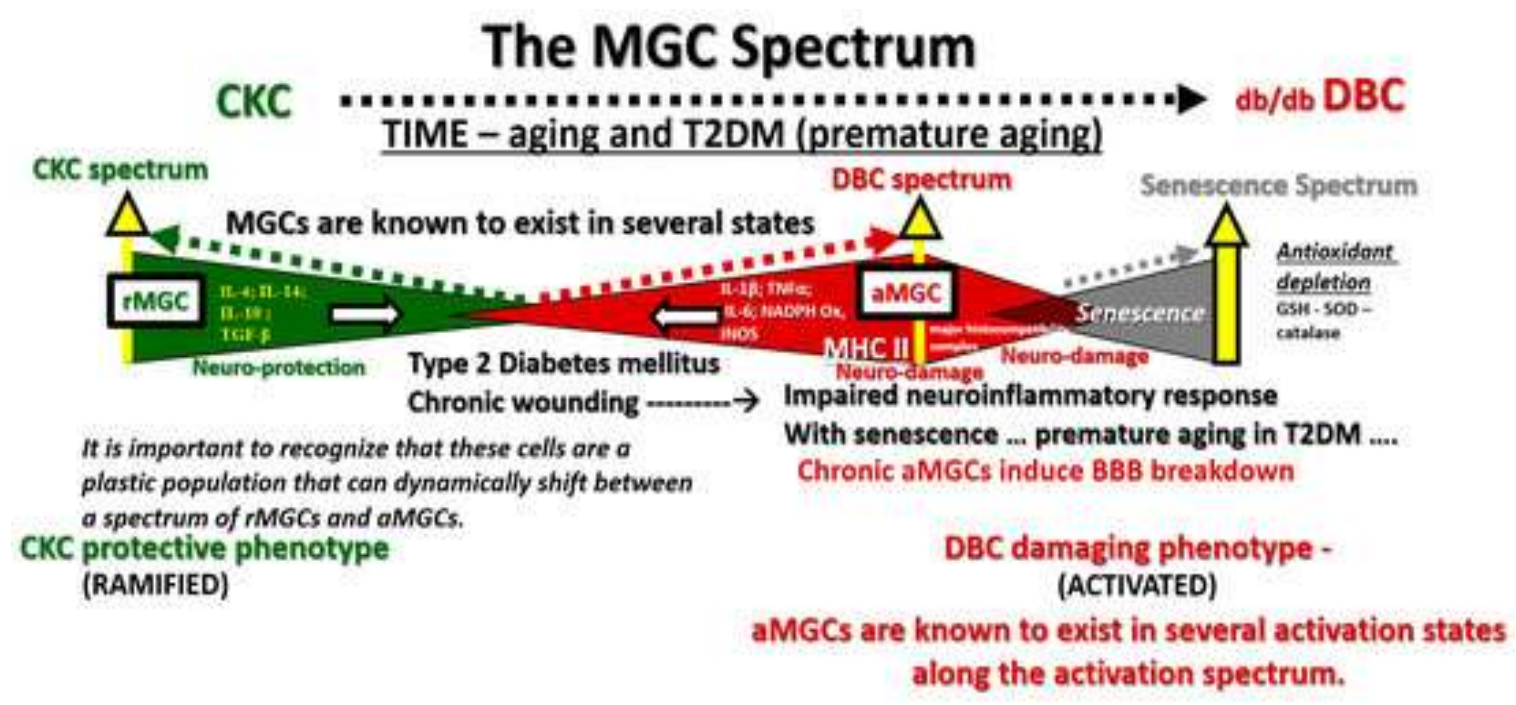

Figure 25. spectrum phenotypes in type 2 diabetes mellitus (T2DM). The microglia cell(s) (MGC) are very unique and capable of undergoing a marked diversity of morphological and functional phenotypic remodeling change as they pass along a spectrum of phenotypes from the ramified MGC (rMGC) (green colorization) on the far left-hand side of this cartoon to the chronically activatedamoeboid microglia cells (aMGC) (red colorization) on the right hand in addition to some microglia progressing to a senescent type of MGC on the far right (grey colorization), which may implicate advanced aging. Note the various cytokines profiles associated with each of the rMGC and the aMGC 
phenotypes. In our age-matched nondiabetic control (CKC) models, the predominate MGC would be rMGC and in the diabetic DBC models the predominant microglia would be aMGC. Note that in health or homeostasis we demonstrate that the MGC may be in a flux or spectral change between rMGCs and more activated/amoeboid MGCs. The regions between open arrows may even represent a range of homeostasis between rMGCs and versatile aMGCs phenotypes, while the dashed lines suggest multiple spectral morphologic phenotypes. $I L-1 \beta=$ interleukin 1 beta; $I L-14=$ interleukin 14 ; IL10 = interleukin 10; TGF- $\beta=$ Transforming growth factor beta; TNF $\alpha=$ tumor necrosis factor alpha; IL-6= interleukin 6; NADPH Ox = reduced nicotinamide adenine dinucleotide phosphate; iNOS = inducible nitric oxide synthase; $G S H=$ glutathione; $S O D=$ superoxide dismutase. Image available via license CC BY 4.0. [5].

Recently, female diabetic $d b / d b$ models were observed to primarily harbor aMGC in cortical layer III in the grey matter in contrast to rMGCs in controls. Additionally, these aMGCs had a marked increase in aberrant mitochondria (aMt) and their nuclei contained a definite increase in chromatin condensation (Fig. 26) [5].

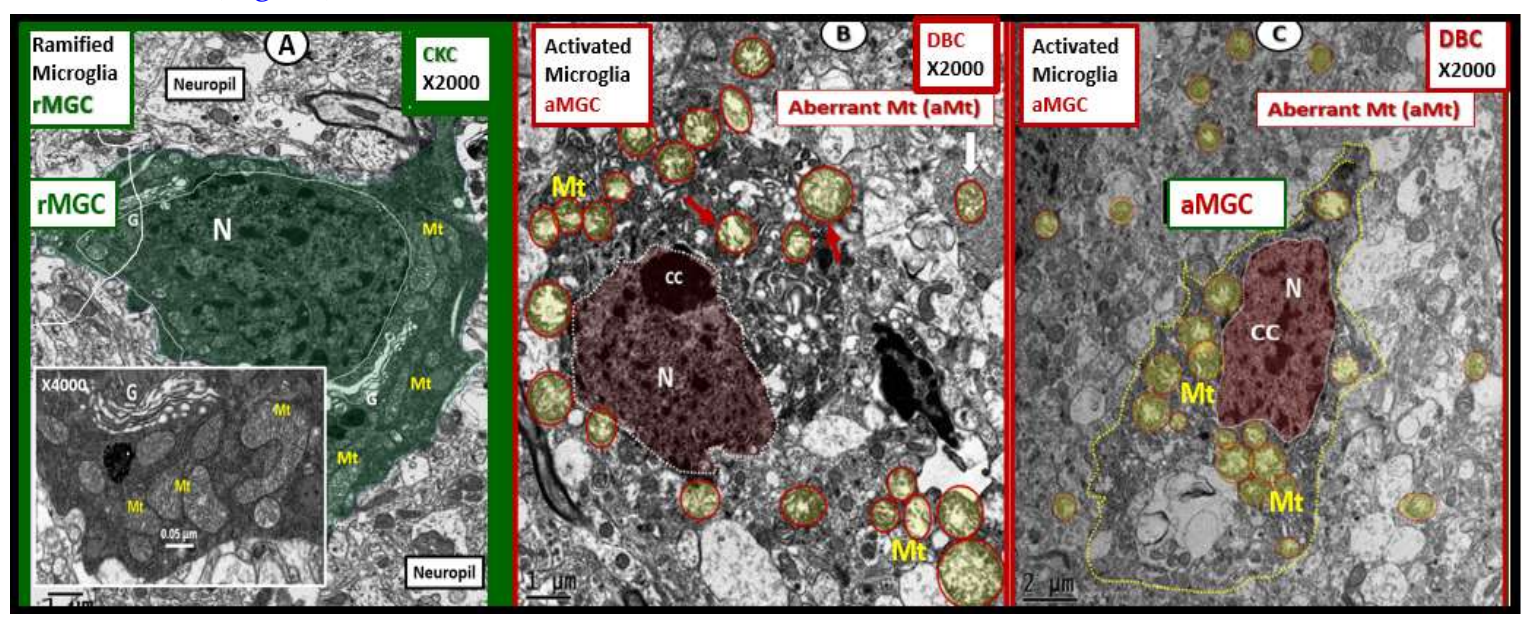

Figure 26. Activated microglia with aberrant mitochondria in fiabetic $\mathrm{db} / \mathrm{db}$ compared to ramified microglia in control non-diabetic models. Panel A illustrates the normal appearing ramified microglia (rMGC) in control CKC. Insert demonstrates the normal electron dense mitochondrial matrix with prominent crista. In contrast panels B and C depict the activated-amoeboid microglia cell (aMGC) a marked increase in aberrant mitochondria (Mt) and also not the nucleus $(\mathrm{N})$ chromatin condensation $(\mathrm{CC})$ in the diabetic DBC models. $G=$ Golgi; $M t=$ mitochondria; $N=$ nucleus. Magnification $\times 2000 ;$ bar = $1 \mu \mathrm{m}$. Exploded insert magnification $\times 4000 ;$ bar $=0.05 \mu \mathrm{m}$. Image available via license CC BY 4.0 [5].

In addition, aMGC were demonstrated to be invasive of the NVU in the diabetic $d b / d b$ models (Fig. 27). 

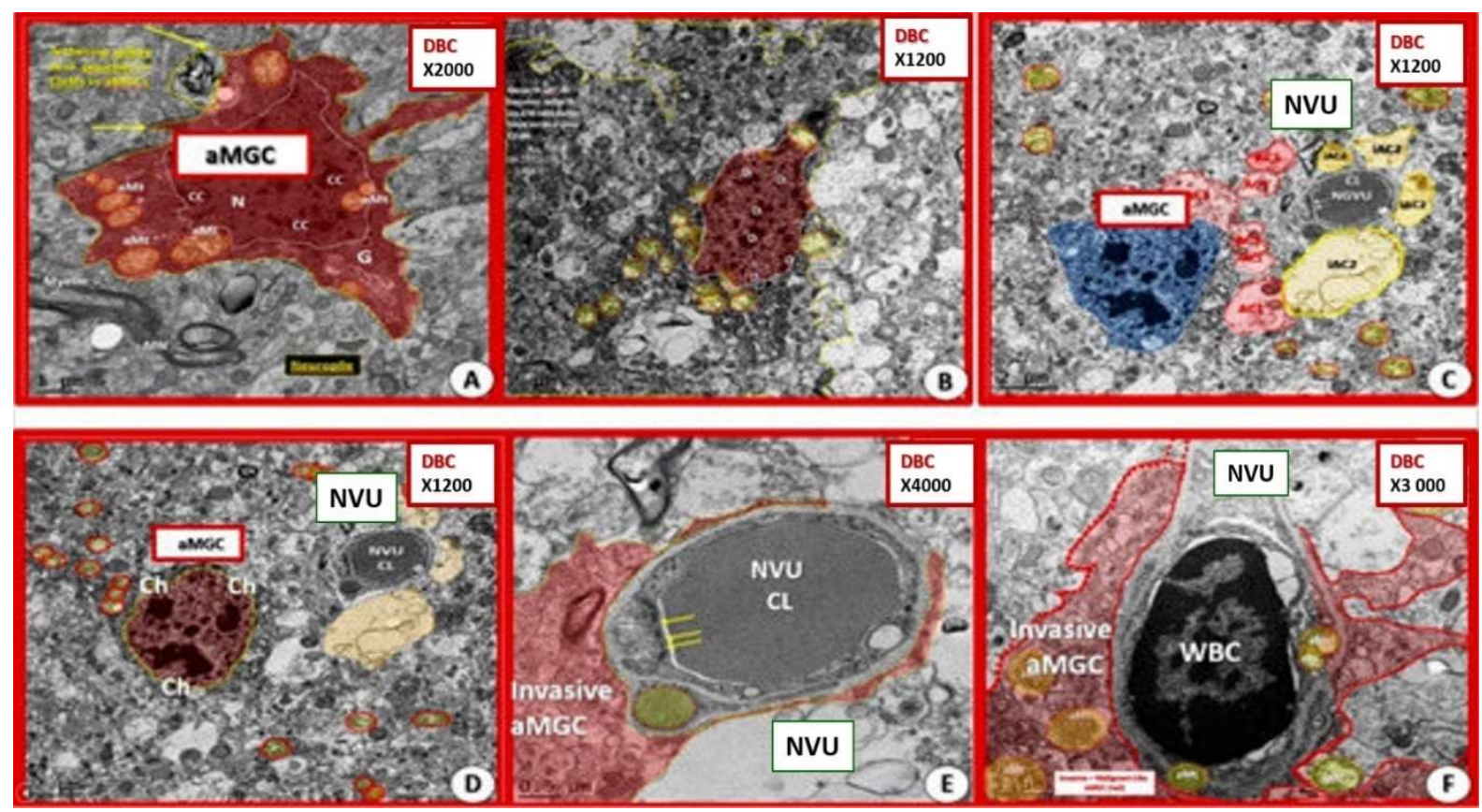

Figure 27. Activated microglia become invasive of the neurovascular unit in diabetic $d b / d b$ models. Panels A and B portray activated microglia cells (aMGC) in diabetic DBC. Panels C,D,E and F depict the invasion of the neurovascular unit (NVU) by aMGCs in DBC. Note the frown-faced chromatin condensation in Panels C and D. Magnification $\times 2000$ Panel A; 1200 Panel B, C, D; $\times 4000$ panel E; $\times 3000$ panel $\mathrm{F}$ with varying scale bars lower left. Image available via license CC BY 4.0 [5].

Importantly, empagliflozin (SGLT2 inhibitor) a glucose lowering treatment for 10 weeks ameliorated these abnormal remodeling changes in MGCs [5].

While the study and knowledge of MGCs is expanding exponentially in regards to neuroinflammation and its relation to T2DM and LOAD, the author would like to direct those with beginning or ongoing interests in MGCs to read the following physiology review by Kettenmann $\mathrm{H}$ et al.[103]. Herein, the following nine postulates from the book chapter "Microglia" by Pio del RioHortega in 1932 still hold true to this very time and will be entered in this section (Table 1) [103, 104].

1) Microglia enter the brain during early development.

2) These invading cells have amoeboid morphology_and are of mesodermal origin.

3) They use vessels and white matter tracts as guiding structures for migration and enter all brain regions.

4) They transform into a branched, ramified morphological phenotype in the more mature brain

5) In the mature brain, they are found almost evenly dispersed throughout the central nervous system and display little variation.

6) Each cell seems to occupy a defined territory.

7) After a pathological event, these cells undergo a transformation.

8) Transformed cells acquire amoeboid morphology similar to the one observed early in development.

9) These cells have the capacity to migrate, proliferate and phagocytose 
Table 1. Pio del Rio-Hortega's nine postulates regarding the microglia from 1932. Please note that number eight is, in compliance with, the recapitulation of embryonic genetic memory upon injury or receiving injury signals such as damage-danger-associated molecular patterns (DAMPs). It is amazing that these nine postulates stand-up to rigorous scrutiny 87 years later.

\subsubsection{Oligodendrocyte and Myelin}

Oligodendrocytes (OLs), oligodendrocyte precursor cells (OPC), and oligodendrocyte lineage cells are specialized glial cells responsible for the synthesis, wrapping ensheathment, and compacting of myelin in myelinated axons $[105,106,107]$. OL derived myelin additionally serves as a protective sheath in myelinated axons in order to provide for long-term axon integrity, maintenance and survival as well as increasing the speed of information transmission from neuron to neuron. Myelinated axons are present in the cortical grey matter layer III; however, they are most abundant within the subcortical white matter, white matter and bundled together to form white matter tracts as in the optic nerves and corpus callosum. These white matter tracts are important for carrying transferring large amounts of information from one region of the brain to distant regions and must rapidly transmit this information. This rapid transmission is primarily due to its compacted electron dense myelin sheaths. As a result, if there is any abnormal remodeling change there may be delays in the arrival of information to the more distant regional neurons with resulting cognitive impairment [108]. Of note, the newer technology of diffusion tensor imaging may lead the way into the future in regards to our better understanding of white matter tract abnormalities in T2DM and LOAD [108]. Certainly, it is already known that prediabetes and T2DM are associated with structural brain abnormalities including lacunar infarcts, white matter hyperintensities (WMHs), cerebral microbleeds (CMBs) and brain atrophy [109].

Previous information demonstrating prominent remodeling changes of oligodendrocytes with increased nuclear chromatin condensation and volume and increased numbers of active myelination sites of the cytoplasm in subcortical transition zones beneath the cortical layers I-VII in the diabetic $d b / d b$ models with known impaired cognition [6. 82]. Marked dysmyelination has also been observed in outer myelin lamellae sheath with splitting, separation, and ballooning with aberrant mitochondria in grey matter and similar myelin remodeling changes with marked myelin disarray and additional axonal collapse in transitional zones in DBC as compared to control CKC models [6], which were protected with the glucose lowering effects of empagliflozin (Fig. 28) [6].

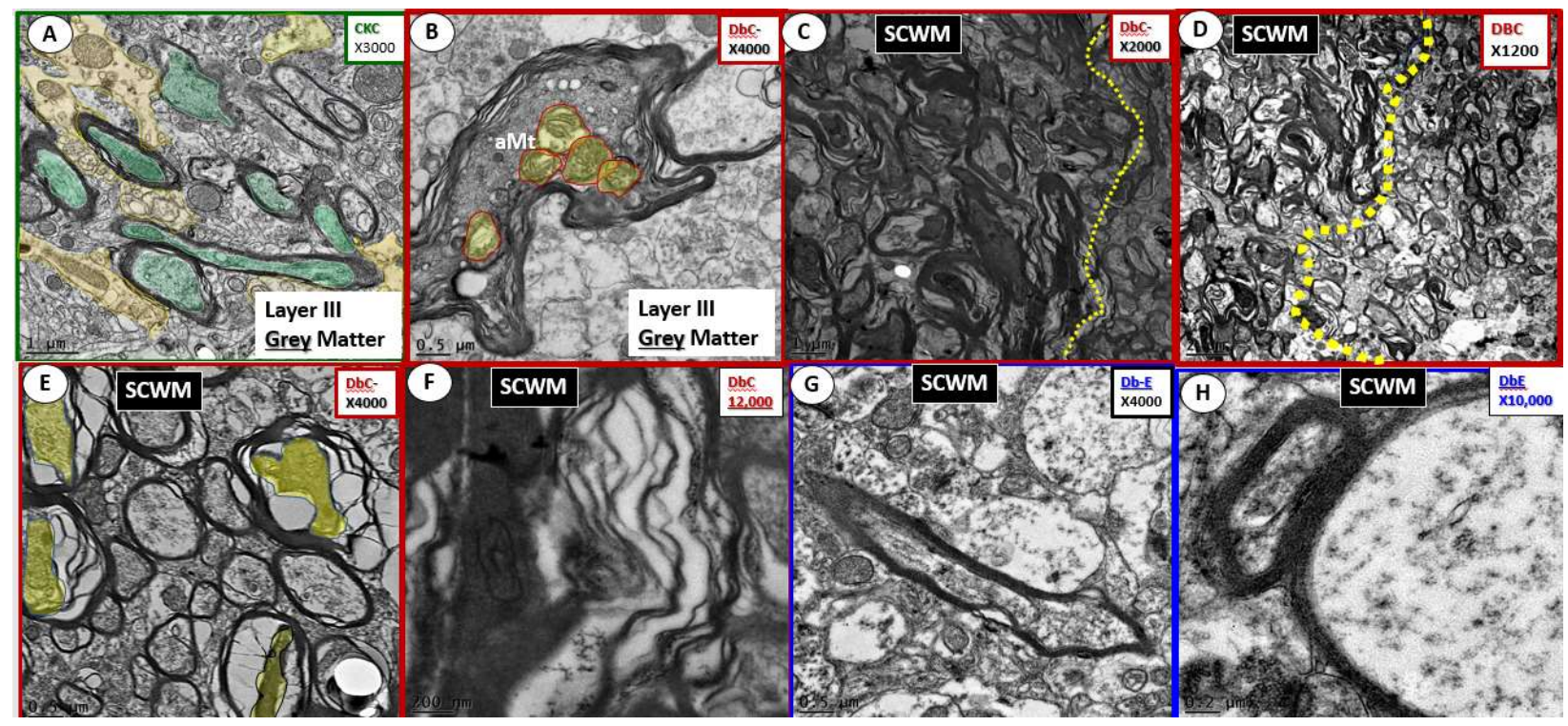

Figure 28. Myelin grey and subcortical white matter comparisons. Panel A illustrates normal electron dense myelin ensheathing axons in Layer III of the grey matter in control CKC models. Magnification X3000; bar $=1 \mu \mathrm{m}$. Panels B-F depict the abnormal myelin remodeling in the diabetic $d b / d b$ (DBC) models with panel B in grey and panels D, E, F, and G in subcortical white matter 
transitional zone just beneath Layers I-VI in the subcortical white matter at various magnifications with scale bar of $0.5 \mu \mathrm{m}$ (B); $1 \mu \mathrm{m}$ (C); $2 \mu \mathrm{m}$ (D); $0.05 \mu \mathrm{m}$ E; $200 \mathrm{~nm}$ (F); $0.5 \mu \mathrm{m}$ (G); $0.2 \mu \mathrm{m}(\mathrm{H})$. $a M t$ =aberrant mitochondria; $S C W M=$ subcortical white matter. Image available via license CC by 4.0 [6].

Additionally, Desai MK et al. have demonstrated that OL/myelin remodeling changes are an early manifestation of triple transgenic $\mathrm{AD}$ mice $(3 \times \mathrm{Tg}-\mathrm{AD})$ as they age and this could possibly also be pertinent to development of LOAD in humans [110]. This possibility may be even more relevant since Met S, obesity, insulin resistance and T2DM are associated with earlier OL/Myelin remodeling in $d b / d b$ diabetic models [6]. Importantly, Ramos-Rodriguez JJ et al., demonstrated gross observable cortical brain atrophy and decreased brain weights in 26-week-old diabetic $\mathrm{db} / \mathrm{db}$ mice [111]. Also, previous MRI studies in T2DM humans have been reported wherein, smaller total brain volumes and cortical grey matter and hippocampal atrophy was evident $[112,113]$. The ultrastructural images in this section and others throughout this review have been from a 20-week-old diabetic $d b / d b$ models.

Even though aging remains the strongest risk factor to develop LOAD and VAD, T2DM remains a risk factor for each of these dementias that indeed may be synergistic via microvascular (NVU) and white matter OL/myelin remodeling.

Earlier in the section 3.2. it was mentioned that there may be three or four barriers provided by the capillary NVU depending if you count the EC BM and the PC inner and outer membrane separately or as continuous BM encasing both the EC and Pc and Pc foot processes (PCfp). Recently, there has been a newer understanding of brain endothelial cells, NVU and BBB, in that, Kutuzov N et al. have examined the penetration of large (40 and 150kDA dextran) and smaller molecular weight sized (376Da sodium fluorescein and 463Da Alexa flor) hydrophobic tracers from carotid artery into the brain to study passive transport from blood to brain [114]. They were able to elegantly demonstrate that the ecGCx is a significant $1^{\text {st }}$ barrier by utilizing fast scanning two-photon microscopy via a cranial window and further, that the EC cytoplasm and its transport systems and adjacent tight and adherence junctions are the $2^{\text {nd }}$ barrier and that the endothelial cell, Pc and Pcfp BMs and ACs create yet a $3^{\text {rd }}$ barrier due to differences in diffusion partition coefficients barrier properties on the brain side of the NVU. Furthermore, they suggested that these three sequences of diffusional constraints or barriers (ecGCx, endothelium and extravascular compartment) be termed the "Tripartite BBB" [114]

These functional diffusional barrier compartments coincide with the ultrastructural findings in this review with the exception that this review initially considered the endothelial BM as its own separate diffusion barrier because it was remodeled and thus four barriers vs. the tripartite barriers by Kutuzov $\mathrm{N}$ et al. Certainly any remodeling changes or alterations to the enGCx, EC and Pc BMs and $\mathrm{ACs}$ as a result of T2DM in previous images would have deleterious consequences to the integrity of the NVU due to a decrease in barrier functions with increased permeability and create an increased cellular vulnerability due to disruptions and loss of integrity to increase the risk and development of LOAD over time and aging.

\section{Oxidative Stress: Reactive oxygen/nitrogen species (ROS/RNS) redox stress and aberrant mitochondria (aMt)}

Aberrant Mt (aMt) were found to be markedly increased in aMGC in the diabetic $d b / d b$ models in section 3.2.6. and were also found to be present in other vascular mural cells (EC and Pc) of the NVU, AC, OL and neurons (myelinated and unmyelinated) in the grey matter cortical regions layer III and the transitional subcortical white matter regions of the diabetic $d b / d b$ brain (Fig. 29) $[4,5,6,7]$. 


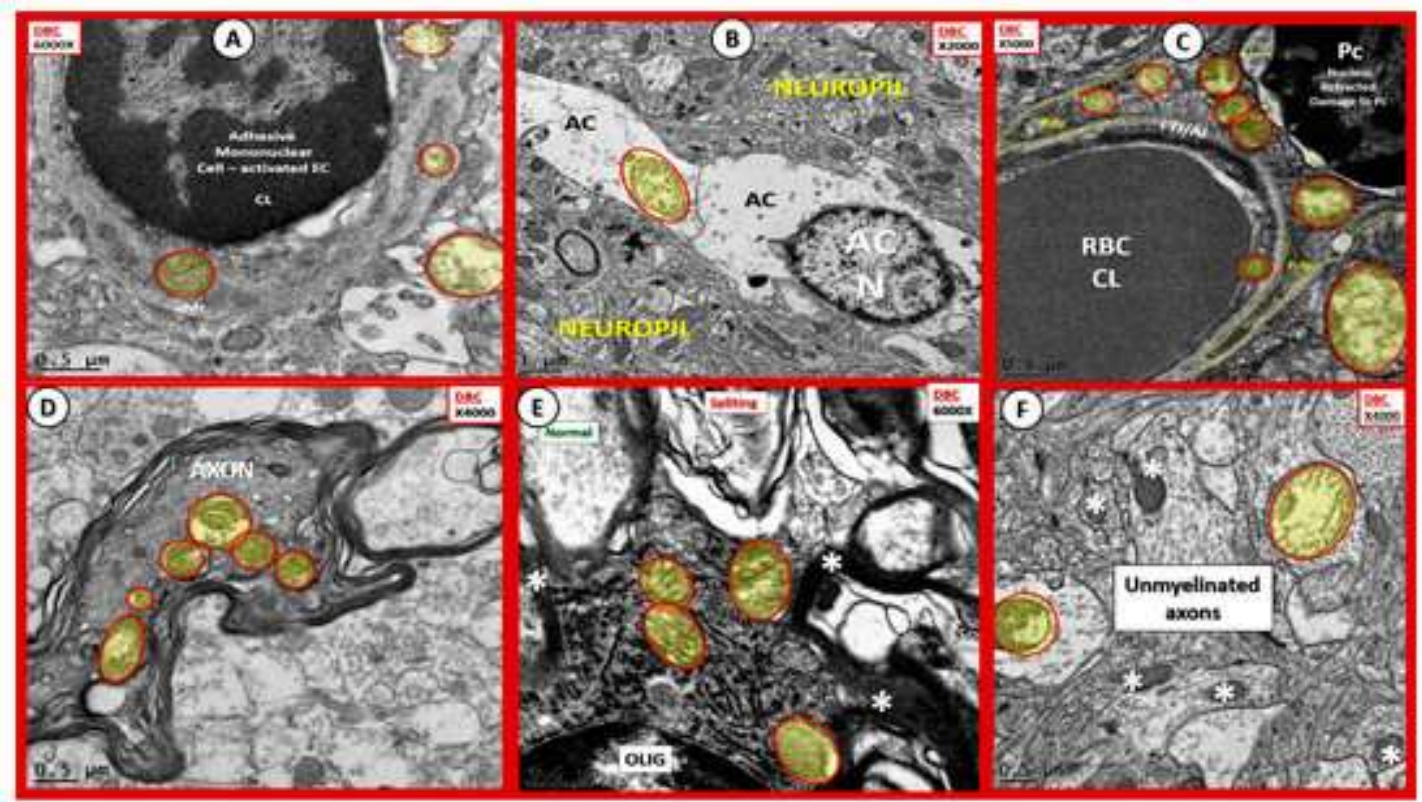

Figure 29. mitochondria in endothelial cells, pericytes and foot processes, astrocytes, oligodendrocytes, myelinated and unmyelinated neurons in the diabetic DBC models. Panels (A-F) demonstrate that aberrant mitochondria $(\mathrm{aMt})$ are found to be present in multiple cells in addition to activated microglia cells (aMGCs). The aMt are pseudo-colored in each of these panels (yellow outlined in red lines) in order to allow rapid recognition. Panels (B,F) are especially important since they demonstrate the aMt characterized by swollen mitochondria (Mt), loss of electron-dense $\mathrm{Mt}$ matrix and crista. Panel (A) illustrates the aMt within the endothelial cells and surrounding aMGC. Panel (B) depicts aMt in ACs. Panel (C) demonstrates aMt in pericytes and foot processes (Pc and Pcfp). Panel (D) depicts aMt in a dysmyelinated neuronal axon. Panel (E) depicts aMt in an oligodendrocyte and Panel (F) illustrates aMt in an AC to the left and an unmyelinated axon on the right within the neuropil. Magnifications are noted in the upper part of each panel and scale bars are located at the lower left-hand side of all panels. Scale bars $=0.5 \mu \mathrm{m}$ in all images except for panel (B) with scale bar $=1 \mu \mathrm{m}$. Image available via license CC by $4.0[4,5,6]$.

Oxidative Stress (ROS/RNS) seems to be of extreme importance at every turn of events or downward arrow from obesity to T2DM to LOAD in the continuum of progression section 3.1. (Fig. 8). ROS beget ROS independently and via previously presented aMGC section 3.2.6. (Fig. 23, 24). The neuroinflammatory aMGCs enzymatic iNOS, NADPH Ox and Mt-derived excessive ROS due to aberrant mitochondria may interact and fuel other sources of ROS/NOS such as: metabolic excess, hormonal excess, renin angiotensin aldosterone system activation, inflammation systemic or neuroinflammatory, hypoxia-ischemia, ischemia-reperfusion, eNOS uncoupling associated with EC dysfunction as in the MetS section1.2. (Fig. 2). These accumulating ROS/NOS of oxidative stress may be synergistic in promoting brain injury with subsequent response to injury remodeling changes in structure and functional abnormalities (Box 3). 


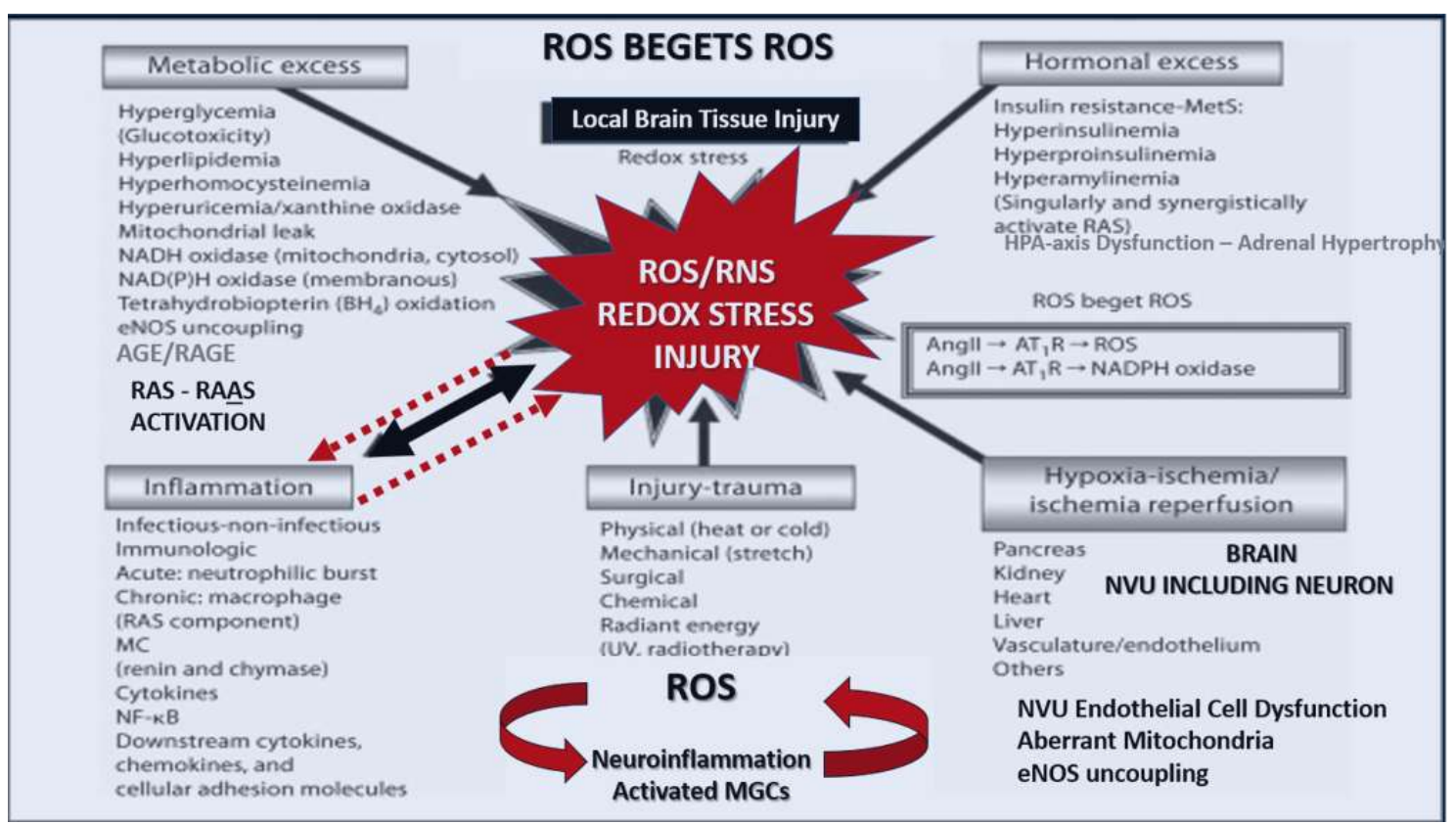

Box 3. ROS beget ROS. This figure illustrates how the metabolic excesses, hormonal excesses of the $\mathrm{H}$ \& E phenomenon interact to produce reactive oxygen/nitrogen species (ROS/RNS) and redox stress. Also note how neuroinflammation, brain injury and hypoxia-ischemia and reperfusion may all work individually or synergistically to produce redox stress damage to the brain resulting in accelerated aging and neurodegeneration. At every downward arrow in the continuum of progression in figure $8, \mathrm{ROS} / \mathrm{NOS}$ and redox stress are involved in the progression of age-related LOAD and support the oxidative stress hypothesis of LOAD. From obesity and insulin resistance of the Met $\mathrm{S}$ to T2DM to NVU remodeling to LOAD. AGE/RAGE = advanced glycation end-products/receptor for advanced glycation end-products; Ang II = angiotensin II; AT1R =angiotensin type 1 receptor; eNOS = endothelial nitric oxide synthase; HPA = hypothalamic pituitary adrenal; $M C=$ mast cell; MGCs = microglia cells; NADPH NADPH Ox = reduced nicotinamide adenine dinucleotide phosphate oxidase; NVU = neurovascular unit; NF$k B=$ nuclear factor- kappa $B ; R A S=$ renin angiotensin system; $R A A S=$ renin angiotensin aldosterone system; ROS/RNS = reactive oxygen species/reactive nitrogen species.

4.1. Oxidative Stress (ROS/NOS): Aberrant Mitochondrial-Derived ROS/NOS Leakage In T2DM EndOrgan Complications

Previous studies have demonstrated an important role of mitochondria in diabetic end-organ complications (Box 4). 


\section{Type 2 DIABETIC COMPLICATIONS - END-ORGAN DISEASE}

\section{Central and Peripheral Nervous System}

a). Diabetic Cognopathy - Encephalopathy

b). VAD, MD, LOAD

cerebral vascular disease,

stroke (occlusive / hemorrhagic) WMH -microbleeds $\mathrm{OL} /$ myelin abnormalities

II. Eye

c). Peripheral Neuropathy

\section{a). Retinopathy \\ b). Cataracts}

III. Cardiovascular

a). Endothelial Cell dysfunction - activation

b). Peripheral Arterial Disease (PAD)

b). Diabetic Cardiomyopathy

c). Coronary Artery Disease - myocardial infarction

d). Accelerated atheroscleropathy - atherosclerosis

e). Vascular arterial stiffness

f). Hypertension

IV. Kidney
a). Nephropathy
b). Glucosuria
c). Proteinuria (microalbuminuria)
d). Renal Failure - end stage renal disease - dialysis

V. Genitourinary

a). Importance

b). Decreased libido - hypogonadism

c). Genitourinary infections (monilial / bacterial)

VI. Skin - Diabetic Dermopathy

a). Infection

b). Impaired wound healing

VII.) Oral disease

a). Caries, gingivitis, periodontal disease, infections VII. Gastrointestinal

a). Delayed gastric emptying - gastroparesis

b). Diarrhea/constipation/dyspepsia

c). Exocrine insufficiency

VIII. Bone

a) osteopenia -osteoporosis: falls and fractures bone mineral quality bone matrix quality even with Bone mineral density is normal

VIII. Foot females $>$ males

a) Diabetic foot

b) Ulceration - amputation

Box 4. Type 2 diabetes mellitus and end-organ remodeling complications. Aberrant mitochondria (aMt) are a central figure in most, if not all, of the end-organ complications. Diabetic end-organs may all have problems with decreased energy production (ATP) and excessive Mt-derived oxidative stress (ROS) production due to abnormalities in structure as well as function. LOAD = late-onset Alzheimer's disease; $\mathrm{MD}$ = mixed dementia; $\mathrm{OL}$ = oligodendrocyte; $\mathrm{VAD}=$ vascular dementia; $\mathrm{WMH}$ =white matter hyperintensities.

In general mitochondria lie at the intersection of many critical cellular pathways involving energy substrate metabolism (ATP generation), calcium homeostasis (acting as a calcium sink), reactive oxygen species (ROS) generation and apoptosis signaling. Furthermore, in healthy cells mitochondria exist as a dynamic reticulum network (in constant fusion and fission) that move around within the cytoplasm assisted primarily by a microtubule actin motor network [115]. Mitochondria are primarily formed within the soma and move down the axon to high energy requiring synapses and if they undergo aberrant mitochondrial (aMt) remodeling changes, they are sent antegrade for repair via (fission and fusion mechanisms) or undergo mitophagy. Thus, if there is impaired autophagy or mitophagy or impairment in microtubule transport due to paired helical fragments and or neurofibrillary tangles even in the early stage of tau formation there may be impaired mitochondrial movement and repair i.e. hyperphosphorylated and aggregated tau appears to damage the axonal transport, leading to abnormal mitochondrial structural defects in synapses [116].

In many of the end-organs that have been previously studied by the author with obesity-genetic or diet induced, insulin resistance, MetS, impaired glucose tolerance or overt T2DM the mitochondria have demonstrated either functional and/or an ultrastructural abnormalities suggesting that oxidative stress may be at the very core of diabetic end-organ complication damage similar to those found in the brain [117, 118, 119, 120, 121, 122, 123, 124, 125, 126, 127, 128, 129, 130]. Thus, ultrastructural aMt in peripheral or central CNS tissues may allow one to strongly suspect decreased ATP generation - hypometabolism, excessive aMt-derived ROS production leakiness and oxidative stress. Of interest, is that in patients and genetic models with LOAD and AD respectively experience mitochondrial dysfunction (aMt) that are associated with bioenergetic deficits and oxidative stress that occur early and may promote $\mathrm{A} \beta$ and Tau pathologies [131].

Nunomura A et al. have demonstrated that oxidative damage is one of the earliest events and furthermore as $\mathrm{A} \beta$ deposition increases and LOAD progresses there is an associated decrease in oxidative damage. They utilized measurements in both neuronal oxidized RNA 8-hydroxyguanosine (8OHG) and the oxidized amino acid nitrotyrosine (3-NT) in LOAD patients. They also demonstrated 
that the 8OHG immunogold studies labeled the cytoplasmic ribosomal endoplasmic reticulum and not the nucleus regions of the neurons. Authors suggested these findings may be due to an increased antioxidant response to $A \beta$ and tau formation [132]. Importantly, if there were chronic ongoing oxidative stress associated with concurrent and/or preexisting ongoing chronic MetS, insulin resistance and T2DM then antioxidant depletion would be more plausible. Swerdlow RH et al. during a decade (2004-2014) have progressed the concept of the mitochondrial hypothesis such that it has now been included in the evolving hypotheses (Box 5) $[133,134,135,136]$.

\section{Major Existing And Emerging Hypotheses for LOAD}

LOAD is an age-dependent, heterogeneous, multifactorial and neurodegenerative disorder functionally characterized early on at its onset by mild cognitive impairment with a progressive cognitive decline progressing to the sixth leading cause of death. Pathologically, LOAD may be characterized by progressive deposition of extracellular amyloid- $\beta$ (1-42) (A $\beta$ ) neuritic plaques derived from amyloid precursor protein (APP), vascular deposition of amyloid- $\beta$ (1-40) in the arteriole vascular media and paired helical fragments (PHF) and neurofibrillary tangles (NFT) derived from abnormal hyperphosphorylation of tau proteins primarily within the neuron.

$\mathrm{A} \beta$ is formed after sequential cleavage of APP by the proteolytic enzymes $\beta$ - and $\gamma$-secretases. The $\gamma$-secretase cleavage at the C-terminal end of the transmembrane region of APP generates a number of isoforms of 36-43 amino acid residues. The most abundant isoforms are $A \beta(1-40)$, which is found primarily in cerebral amyloid angiopathy and $A \beta(1-42)$ in neuritic plaques. Also varying lengths of oligomers of $A \beta$ have been found to be the toxic form of $A \beta$ rather than the $A \beta$ neuritic plaques [137].

Tau is a neuron microtubule associated protein that is essential for proper function. The microtubules are associated with the cytoskeletal actin motor unit elements of neurons and are essential for the mobility of neurotransmitter vesicles and mitochondria cellular movement along with other organelles within neurons during their long passage from the soma to the dendritic synapses to provide synaptic transmission from one neuron to another. In LOAD tau becomes hyperphosphorylated and is known to disrupt normal microtubule functions with impaired transport of neuronal cellular organelles to dendritic synapses and if necessary, to transport damaged mitochondria antegrade to the soma regions to undergo macro-autophagy or mitophagy. Tau is thought to become hyperphosphorylated and in this hyperphosphorylated state it becomes polymerized into paired helical filaments (PHF) admixed with straight filaments, which ultimately form neurofibrillary tangles (NFTs) and the second hallmark lesion of misfolded proteins along with $\mathrm{A} \beta$ associated with LOAD. [138]. Currently it is thought that $\mathrm{A} \beta$ formation and deposition occurs earlier than the PHF and NFT of tau. Additionally, PHF and NFT of misfolded tau correlate more closely with the clinical progression of the disease in $\operatorname{LOAD}[137,138]$.

Over the last century, since Alois Alzheimer made his initial discovery in a younger early onset 50-year-old female patient suffering from dementia in 1906, there have emerged numerous hypotheses regarding the development of LOAD [139]. These numerous major and emerging hypotheses (Box 5) have developed from many different laboratories and multiple researchers and will undoubtedly continue to grow and/or change as new information comes forward but for now these are currently felt to be the best candidate hypotheses, which we have attempted to illustrate and discuss in the important intersects between T2DM and LOAD in section 2. 


\section{Major Existing And Emerging Hypotheses for LOAD}

I. AGING Hypothesis: Considered the greatest risk factor for the development of LOAD

II. Cholinergic Hypothesis: The earliest hypothesis and generated our first clinical treatment with anticholinesterase medication.

III. Ap cascade Hypothesis; has been the mainstay hypothesis for at least 2 decades. A Irigger that may be necessary but not sufficient (see DX. Vascular 2 hit hpothesis).

IV. Tau Hypothesis: neuronal loss atrophy. Insulin resistance of importance in relation of dysfiunctional glycogen synthase kinase (GSK3B) signaling, which allows for hyperphosphorylation of Tau and misfolding aggregation of tau proteins with malfunctioning of microtubules with disturbance of neurotransmitter carrier and Mt energy source carrying functions.

\section{$* * *$ HUGH intersect with obesity, insulin resistance and T2DM - LOAD and \\ Traumatic brain injury (TBI) via GSK3 3 intersect}

V. Inflammation Hypothesis; Reactive gliosis and neuroinflammation are hallmarks of $A D$. Microglia activation. In response to multiple stressors i.e. oxidative stress $-\mathrm{ROS}$ begets inflammation and inflammation begets ROS. Toxic cytokine release ROS. MGC activation thus far in all models examined: Western Brain. TIDM streptozotocin models, and especially T2DM i.e. obesity, Insulin resistance and T2DM db/db and BTBR ob/ob models thus far studied.

VI Oxidative Redox Stress Hypothesis: *HUGE intersect with LOAD and may be the greatest and one of the earliest intersects bit T2DM and LOAD.

ROS beget ROS aMt beget aMt via Neuroinflammation aMGCs and toxic cytokines.

VII. Hypometabolism Hypothesis: Glucose Metabolism Hypoglycemia and Hyperglycemia. For example, glucose metabolism brain imaging such as "FDG-PET (Positron emission tomography with 2deoxy-2-fluorine-18-fluoro-D-glucose) has become valuable indicator for diagnosis of neurodegenerative diseases that cause dementia, including $\mathrm{AD}$. Dysfunctional Mt are a central and important finding in diabetic brains. Of importance is that BOLD PEI scans and studies contribute data that are in addition to measuring the amount of glucose delivered as it also measures the CBF since the vasculature delivers the glucose that is delivered and measured.

VIII. Genetic Hyporhesis: AreE-24 and various genetic single nucleotide polymorphisms. SNPs and possibly includes maternal mitochondria inheritance by the genetic hypothesis.

IX. Vascular Hypothesis - 2 Hit Hypothesis and the (micro)-vascular cognitive impairment and dementia (VCID) Hypothesis: These paired hypotheses have merged into accepted hypotheses in the present time not only for vascular dementia (VAD) but also late-onset Alzheimer's Disease (LOAD).

$X$. Evolving Hypotheses:

1. Mitochonarial Cascade Hypothesis: Rapidly emerging as a sustaining hypothesis.

2. Microbiota-gut-brain-immune axis Hypothesis: Most certainly this hypothesis will be equally accepted (as I-IX) over time and is definitely an exciting story to observe. Rapidly emerging as a sustaining hypothesis.

Box 5. Major existing and emerging hypotheses for late-onset Alzheimer's disease (LOAD). AD = Alzheimer's disease; APOE- $\varepsilon 4$ = apolipoprotein E epsilon 4; BOLD = blood-oxygen-level-dependent imaging; $\mathrm{CBF}$ = cerebral blood flow; $\mathrm{LOAD}=$ late-onset Alzheimer's disease; $\mathrm{MGC}=$ microglia cell; $\mathrm{PET}$ = positron emission tomography; ROS = reactive oxygen species; T1DM = type 1 diabetes mellitus; $\mathrm{T} 2 \mathrm{DM}=$ type 2 diabetes mellitus; $\mathrm{VAD}-\mathrm{VaD}=$ vascular dementia.

\subsection{Endothelial Cell Activation - Dysfunction And Impaired Cerebral Blood Flow (CBF) In Diabetic db/db} Models: Hypometabolism (VII) and Vascular (IX) Hypotheses Elaborated

The brain receives $20 \%$ of the cardiac output and also $20 \%$ of the body's oxygen and glucose in order to maintain proper homeostasis. CBF is responsible for the delivery of essential oxygen and glucose and therefore healthy blood vessels are essential and if interrupted, damage may occur to cerebral neurons in minutes. This estimated 400-mile trek of capillary NVUs allows the cellular 
elements to be perfused and when there is increased neuronal activity this neurovascular coupling allows for increased CBF to provide the necessary nutritional supply of glucose and oxygen, which is dependent on intact NVU coupling and functional hyperemia [140]. Thus, if there is any dysfunction or interruption of CBF to supply the increased neuronal activity the neurons suffer initially with dysfunction and later neurodegeneration due to oligemia - ischemia.

EC function plays a huge role in maintaining the competence of NVU coupling and CBF in the brain via functional hyperemia. EC dysfunction or activation is associated with impaired NO production via eNOS enzyme uncoupling due to oxidative stress, decreased tetrahydrobiopterin (BH4), increased vasoconstrictors such a endothelin-1 (ET-1), angiotensin II (Ang II) and disturbed regulation of inflammation and thrombosis. Since NO is known to be an inhibitor of platelet aggregation and leukocyte adhesion, EC activation has detrimental effects on cerebrovascular - NVU functions and CBF [141]. EC dysfunction - activation (Fig. 2) was presented in section 2. and is known to be present as an early abnormality in MetS and T2DM. EC activation in the NVU due to impaired nitric oxide generation and eNOS enzyme uncoupling with decreased NO bioavailability could contribute to NVU uncoupling and decreased CBF [141].

Altered platelet, red and white blood cell rheology is known to be present in T2DM [142]. Adherence of activated platelets, erythrocyte and leukocytes are known to be associated with EC activation in preclinical models and humans with T2DM $[143,144]$. Previous studies in the diabetic female T2DM $d b / d b$ models have demonstrated major remodeling changes to the EC as a result of EC activation in the NVU, which consisted of platelets, red blood cells (RBCs), leukocyte adherence in addition to the well-known fibrin network dysfunctional abnormalities (Fig. 30).

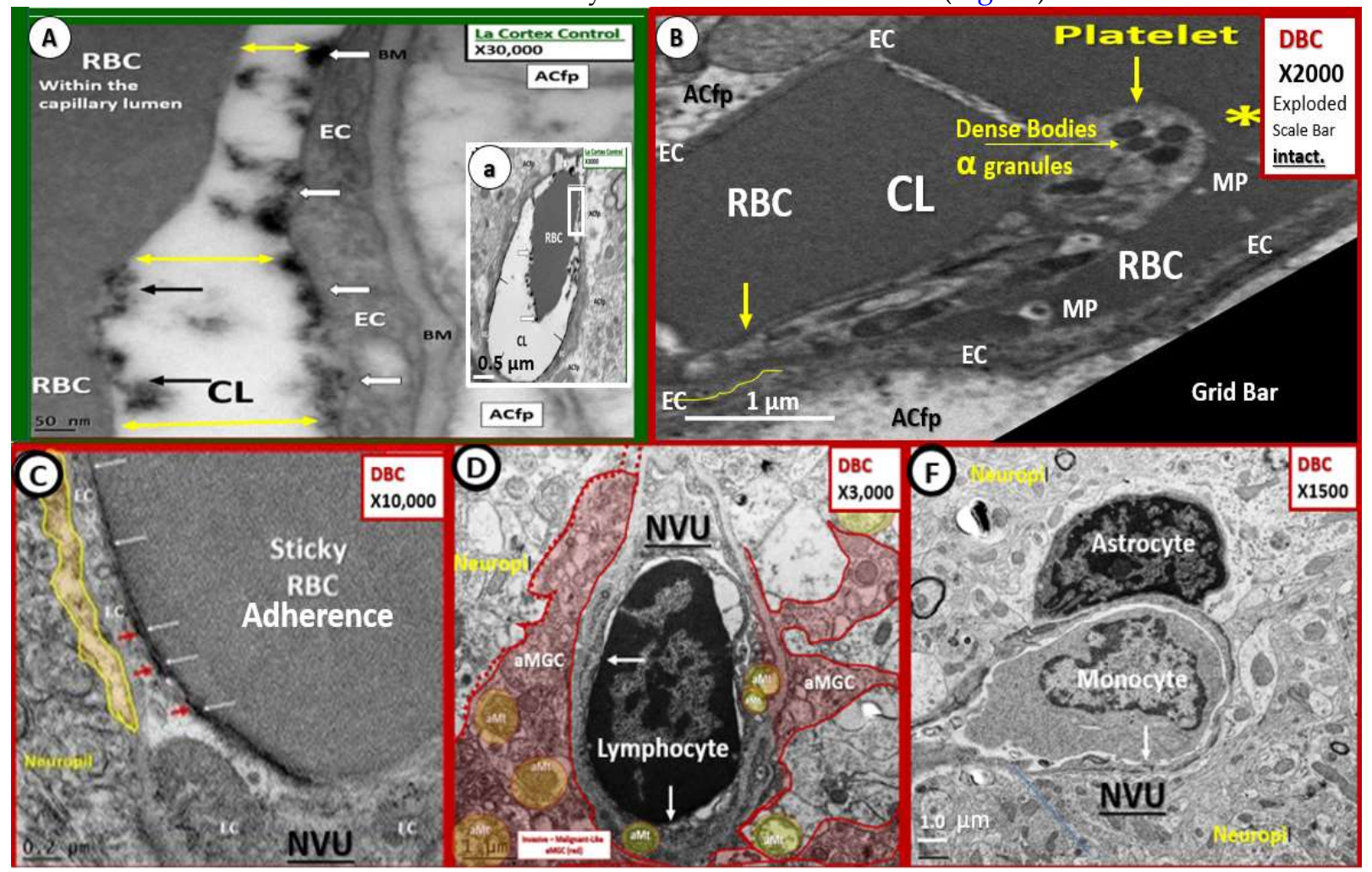

Figure 30. Adherent red blood cells, leukocytes and activated platelets to activated endothelial cells in $d b / d b$ Models. Panel A depicts an intact endothelial glycocalyx (ecGCx) on both the intraluminal red blood cell (RBC) and the luminal surface of the endothelial cell (EC) with lanthanum nitrate staining (arrows) with magnification x30,000; scale bar $=50 \mathrm{~nm}$ in a non-diabetic control C57B6 mouse model. Insert (a) demonstrates this same capillary neurovascular unit (NVU) with intraluminal RBC with lower magnification $x 3000$; scale bar $=0.5 \mu \mathrm{m}$ to depict the entire capillary NVU. Note that the EC and RBC are each decorated by an extremely electron dense protective surface coating of an ecGCx (arrows) that are separated and not adherent in control models with pre-sacrifice lanthanum nitrate infusion that has been found to be lost or shed in diabetic models (such as in the $\mathrm{db} / \mathrm{db}$ and ob/ob models - not shown). Panel B depicts an adherent activated platelet (arrows and yellow line lower 
left) to the EC and note the elongation of this platelet suggesting activation in addition to its adhesion to the capillary NVU endothelial cell. Magnification x2000 enlarged in paint program with intact scale bar $=1 \mu \mathrm{m}$. Panel C illustrates adhesion of a RBC to the EC in the $d b / d b$ diabetic DBC model with an electron dense adhesion plaque (electron dense (arrows) of the NVU. Magnification x10,000; scale bar $=0.2 \mu \mathrm{m}$. Panels D and F depict the tight adherence of leukocytes to the endothelium (adherence points depicted with arrows) in the diabetic $d b / d b$ DBC models. A lymphocyte in panel D and a monocyte in panel F. This tight adherence of RBC and leukocytes and activated platelet are representative of an activated endothelium in the diabetic DBC models. Various magnifications utilized to demonstrate the adherence. Magnification $\times 3000$; bar $=1 \mu \mathrm{m}$ (panel D); magnification $\times 1500$; bar $=1.0 \mu \mathrm{m}$ (panel F). Panels C and D available via CC by 4.0. [4].

The adhesion of RBCs, leukocytes and activated platelet to the NVU activated endothelium observed in the obese insulin resistant diabetic $d b / d b$ DBC models as observed in figure 33 would definitely result in increased inflammation and oxidative stress but equally important would be their physical obstruction to $\mathrm{CBF}$ and decreased distal perfusion and resulting ischemia to neurons due to a stalling of CBF.

Just as there were adherent and stalled neutrophils in the study by Cruz Hernández JC et al. the $d b / d b$ DBC diabetic mice have demonstrated adherent stalled RBCs, leukocytes and platelets. So in addition to the impaired CBF that may be associated with AC retraction and loss of neurovascular coupling and impaired vascular hyperemia there may also be stalled RBCs and leukocytes due to adhesion to an activated and dysfunctional endothelium with adherent activated platelets. As a result of our findings regarding the AC detachment and adhesion of RBCs and leukocytes in the diabetic $d b / d b$ models we can now demonstrate at least two morphological ultrastructural changes in the $d b / d b$ that could interfere with CBF that appear to be protected with empagliflozin [7]. This type of oligemia and decreased CBF in the $d b / d b$ diabetic mouse model would certainly accelerate the remodeling changes and contribute to the increased risk that would be associated with a pre-existing T2DM on the age-related development of LOAD. Additionally, impaired CBF as measured by cerebral glucose metabolic rate (CMRglu) by fludeoxyglucose F 18-positron emission tomography (FDG-PET) are known to be associated with LOAD risk and even observed prior to the onset of dementia $[145,146,147,148]$. Thus, brain hypoperfusion and impaired CBF may participate in the pathogenesis/pathophysiology of neurodegenerative diseases such as in LOAD with underlying T2DM.

A brief synopsis of some of our findings certainly tie into the preceding paragraphs in that, the five major conditions - categories as listed in the abstract consisted of $i$. aging; ii. metabolic (hyperglycemia and advanced glycation end-product and receptor AGE/RAGE interaction resulting in oxidative stress and inflammation in addition to insulin resistance as a linchpin between T2DM and LOAD; iii. oxidative stress; iv. inflammation; $v$. vascular (extra and intracranial macrovascular, vascular stiffening and NVU microvascular disease) all come into play in discussing the increased risk of LOAD with underlying T2DM and the phenomenon of impaired CBF. Additionally, in the $d b / d b$ (DBC) diabetic model we have learned that there is microvascular disease of the NVU with EC activation with $\mathrm{RBC}$ and $\mathrm{WBC}$ adherence resulting in decreased CBF due to cellular adhesion resulting in stalling of $\mathrm{CBF}$. Additionally, attenuation and/or loss of the BBB TJ/AJ, thickening of the $\mathrm{BM}$, detachment of the $\mathrm{AC}$ with impairment of functional hyperemia and neurovascular uncoupling between active neurons and dilation of the NVU in T2DM could markedly increase the vulnerability of these cells as age-related LOAD begins to develop and would contribute to the brain atrophy noted by Ando Y [67].

\section{Neuropathological changes in LOAD}

The neuropathologic substrate of LOAD consists of extracellular neuritic plaques (A $\beta$ 1-42) and CAA (A $\beta 1-40)$ and intracellular-intraneuronal hyperphosphorylated misfolded microtubuleassociated tau proteins [149]. Despite all of the newer sophisticated diagnostic procedures and tests, autopsy and neuropathological examination still remains the 'gold standard' for the definitive diagnosis of LOAD. The only visible gross finding in LOAD is cortical atrophy and enlargement of 
the lateral ventricles; however, this is not specific for LOAD and could include many other neurodegenerative diseases, but this finding certainly raises a red flag to the neuropathologist. Numerous regions within the brain are affected by the neuritic plaques and neurofibrillary tau tangles; however the entorhinal cortex, the CA1 and subicular regions of the hippocampal regions are in particular heavily involved along with other regions such as the amygdala, and the deeper layers (layers III, V, and superficial VI) of the neocortical regions of the brain. The extent and distribution of neurofibrillary tangles in cases of LOAD correlate with both the degree of dementia and the duration of illness as well as staging [150] and have been thought of as one of the cardinal histopathological lesions along with neuritic plaques of LOAD. There has also been demonstrated to be a synaptic loss of neurons in LOAD (up to $45 \%$ ) and this finding is important to the neuron to neuron communication and synaptic loss is felt to contribute to the cognitive impairment found in these individuals along with cerebral atrophy. Even though LOAD is the sixth leading cause of death, it is often not the direct cause of death and many of these patients may die from other associated diseases such as sepsis, pneumonia, traumatic falls with injury and cardiovascular diseases including stroke due to the older age of these patients with multiple comorbidities and frailties. The most commonly associated condition with LOAD is stroke and ischemic infarction. Additionally, another co-morbid condition, Parkinson's disease with or without Lewy bodies, is often associated with clinical diagnosis of LOAD at the time of neuropathologic study. While the above may be somewhat of a superficial sketch of the neuropathological findings, it does add to the overall knowledge and background of this important topic [149]. These previous observations speak out strongly for the importance of mixed dementias as discussed in section 2. (Fig. 6).

\section{Discussion and Concluding Remarks}

T2DM is known to be associated with macro and microvascular complications in the peripheral vasculature such as in accelerated atherosclerosis, peripheral neuropathies, retinopathies, nephropathies and cardiomyopathies for many years. In contrast the central brain has been studied as an end-organ for a much briefer time period. We are now gaining knowledge rapidly and still learning on a regular basis regarding the various mechanisms of functional and structural change that occur in the brain end-organ of T2DM. Although, the mechanisms implicated have not been completely elucidated it is now known that cognitive impairment, $\mathrm{VaD}$, stroke, anxiety/depression and LOAD have been related to T2DM. This knowledge, plus the role of the macro and microvessels being the provider of essential oxygen and nutrients and to dispose of or efflux metabolic byproducts of metabolism are all important. Additionally, astrocytes and their very unique ultrastructure and functions of being connected and signaled by high activity of regional neurons providing neurovascular coupling and functional hyperemia to increase cerebral blood flow in times of need are essential for proper brain perfusion. These various cells and functional substructures such as the NVU with their readily identifiable ultrastructure has directed this review to heavily focus on both its structure and its function in order to autoregulate its vascular supply. Prior to this time the BBB along with its necessary TJ/AJ held the role of protecting the neurons but now we understand that there is more to this highly dynamic structure as we study the NVU with the many cells it employs in order to undergo a great deal of crosstalk between one another. Once this important NVU structure is injured and remodeled by T2DM, the neurons may be deficient of proper function and allow dysfunctional remodeling change with synaptic dysfunction, loss and eventually neuronal dysfunction and loss with neurodegeneration and atrophy.

$\mathrm{LOAD}$ and $\mathrm{VaD}$ are the most frequent contributors to cognitive dysfunction and dementia. Further, they frequently and commonly co-occur as a mixed dementia (MD) in autopsy studies. The risk for stroke or dementia is great for both sexes, i.e. at age 65, one out of four men and one out of three women will have an increased risk of developing stroke, dementia or both [151]. Additionally, when the first baby boom generation began turning 65 years of age at a rate of approximately 10,000 per day in 2011, they were estimated to be just under 77 million strong in the United States population [https://www.census.gov/prod/2014pubs/p25-1141.pdf] accessed July 10, 2019. 
T2DM and LOAD are two independent chronic age-related diseases that are each associated with high morbidity and mortality in our current aging global societies. Since both are age-related and chronic diseases, it is not unusual for them to co-occur along with cerebro-cardiovascular disease and LOAD. Both have multifactorial causations and risk, in which, there are at least 18 intersects (Box 2) with insulin resistance, deficiency and/or insensitivity emerging as a linchpin between the two disparate diseases. In fact, as these two diseases merge in our aging society they may form a troublesome excess or a "bottleneck" of senior citizen's over the next two decades due to the worldwide aging societies. This conundrum will undoubtedly create a strain on our health care system, a financial burden to our society and much stress to individual caregivers and families. A great deal still remains unknown; however, a wonderful discussion regarding what is and what is not known regarding brain insulin resistance can be found and is strongly suggested [152, 153. 154].

As previously discussed in section 1.2.1 peripheral insulin resistance is a definite core feature of T2DM that is rapidly emerging as a core feature of LOAD as brain insulin resistance [155]. BIR may be defined as the failure of brain cells to respond to insulin [154, 155].

In the near future we will come to better understand BIR, since intranasal insulin is to be studied in human clinical trials [156]. Already, it has been determined that intranasal insulin has been able to not only improve multiple systems that are known to be involved with LOAD like impaired glucose uptake but also improve memory, focus and activities of daily living in multiple studies without causing hypoinsulinemia [157]. Six intranasal insulin treatment trials have been rigorously reviewed in regards to MCI and LOAD by Avgerinos KI et al. [158, 159, 160, 161,162, 163, 164].

The evidence continues to grow stronger that there is impaired insulin signaling (brain insulin resistance) in the brains of those human individuals with LOAD. Additionally the combination of T2DM and LOAD suggest that the two disparate diseases may be synergistic when they co-occur and demonstrated the greatest decrease in insulin signaling proteins [164]. Recently, the brain insulinPI3K-AKT signaling pathway from autopsied frontal cortices (nine with LOAD, 10 with T2DM, eight with T2DM and LOAD were compared to seven control human autopsies) and found that the greatest PI3K-AKT signaling impairment was more severe in individuals with both T2DM and LOAD [164]. Further, this group found in T2DM and LOAD patients there was a decrease in insulin receptors, IRS1 total, PI3K(85) total - phosphorylated (p)PI3K(p85), PDK1total - (p)PDK1, AKT total - (p)AKT, GSK-3 $\beta$ total - (p)GSK-3 $\beta$ (allowing hyperphosphorylation of tau and PHF and NFT) and increased Calpain-1, which could allow for increased degradation of these decreased insulin signaling proteins [164]. Also, this group found that there were decreases in glucose transporters, namely GLUT1 (endothelial GLUT) and GLUT3 (neuronal GLUT) as well as decreased HIF-1 and O-GlcNAcylation, which correlated with increased hyperphosphorylation of tau in short time post-mortem AD brains ( $<3$-hour average postmortem delay). These findings strongly support the notion that GLUT1 and GLUT3 deficiencies could cause impaired brain glucose uptake and/or metabolism, which could contribute to neurodegeneration in correlation to a decrease in O-glcNAcylation and hyperphosphorylation of tau in AD [165].

The following two additional emerging hypotheses may be 'game changers' in regard to both the novel treatments of LOAD by repurposing insulin and insulin sensitizing medication and aiding in the understanding of the development and/or the continuum of progression in LOAD.

\subsection{Metabolic Hypothesis: Hyperglycemia and Hyperinsulinemia}

Hyperglycemia - glucotoxicity and hyperinsulinemia of T2DM and MetS with peripheral insulin resistance (PIR) and central - brain insulin resistance (BIR) may be extremely injurious to brain metabolic functioning and brain end-organ remodeling associated with the increased risk in the development of LOAD (Box 1), section 1.2.1 [166].

The importance of this evolving hypothesis allows one to consider the possible impact of excessive amounts of amyloidogenic amylin (islet amyloid polypeptide - IAPP) - hyperamylinemia to be deposited in the brain's perivascular and extracellular matrix. Amylin is co-synthesized, copackaged and co-secreted from the islet pancreatic beta cell's insulin secretory granule due to peripheral insulin resistance (PIR) $[167,168]$. Amyloidogenic amylin may be deposited as amyloid 
amylin $(\mathrm{A} \alpha)$ and eventually function as a possible niche region in the brain for cross-seeding with $\mathrm{A} \beta$ and allow $\mathrm{A} \beta$ and $\mathrm{A} \alpha$ to undergo mixed perivascular and extracellular co-deposition in addition to $\mathrm{A} \alpha$ independent deposition in the perivascular regions and extracellular matrix, which may possibly interfere with $A \beta$ efflux or clearance $[169,170,171,172,173,174]$. The amylin hypothesis involving the amyloidogenic A $\alpha$ might also lead to the use of an amylin analog (pramlintide) for clinical study that has already been approved and utilized to treat type 1 and T2DM and known to improve glucose levels [175]. Incidentally, pramlintide has been reported to improve the feeling of "well-being" by those having used it in the original treatment trials (authors personal communication in 2004) [176].

Additionally, fibrinogen is one of the biomarkers of inflammation and an important risk factor for many cardiovascular and cerebrovascular diseases including T2DM and LOAD [177]. Misfolded proteins and their deposition are also important to the development of LOAD. Additionally, this concept of misfolded proteins allows for the fifth misfolded protein in LOAD that being amyloid fibrinogen-fibrin (AFib) due to activated platelets and its membrane amyloid precursor protein, which contribute to $A \beta$ deposition and known to be associated with MetS and T2DM [178]. Currently, there are at least five known and distinct misfolded proteins consisting of $A \beta(1-40), A \beta(1-42)$, tau (NFT), amyloid amylin (A $\alpha$ ) and now AFib that contributes to $A \beta(1-42)$ via the activated platelets, which increase the amyloid precursor protein and AFib. In the future, there may be even more misfolded proteins found to be involved in the development of LOAD.

\subsection{The Antimicrobial Protection Hypothesis: Antimicrobial peptide(s) (AMP)}

$\mathrm{A} \beta$ is known to be an antimicrobial peptide [179]. Previous infections such as Human alphaherpesvirus 1 (HSV-1 - viral), Chlamydia pneumoniae (bacterial), Borrelia burgdorferi or recurrentis (spirochete) plus other pathogens or the ongoing chronic sterile inflammatory process (activated MGCs) have all been implicated in activating AMPs [178 179]. Interestingly, this infectious hypothesis or antimicrobial protection hypothesis is somewhat reminiscent of the Helicobacter pylori (gastritis and duodenal ulcer story) and certainly an emerging hypothesis to follow closely, while keeping in mind the potential of antiviral and antibacterial drug repurposing $[180,181]$. One of the antibiotics that the author is interested in following is minocycline, because it is known to pass the BBB and is also known to be a matrix metalloproteinase inhibitor [182].

The ongoing chronic sterile inflammatory process (activated MGCs) as occurs in LOAD and T2DM may result in excessive AMP-APP production of $A \beta(1-42)$ due to activation of excessive APPs. The chronic sterile inflammatory process may be perceived as a pathogenic-like threat, which results in increased APP synthesis and cleavage to oligomeric forms of $A \beta(1-42)$ to form amyloid fibrils in order to entrap pathogens and serve as an innate immune pathway as a response to a perceived active infection or a sterile inflammatory response. This process could act in a feed-forward viscous cycle to result in ongoing $A \beta(1-42)$ deposition of extracellular neuritic plaques. This emerging antimicrobial protection hypothesis does not interfere with the existing $\mathrm{A} \beta$ cascade hypothesis in Box 5 (III), Section V; however, it does add a novel dimension to this exiting hypothesis and may suggest that an innate immune-mediated inflammation could propagate LOAD A $\beta(1-42)$ deposition and ongoing neurodegeneration [183, 184, 185]. Additionally, this could allow for the consideration of antibacterial, anti-spirochetal and antiviral treatments if an underlying source of infective agent could be identified via past medical history of previous infections or current diagnostic findings.

Historically, the NVU concept emerged and was formalized at the 2001 Stroke Progress Review Group meeting of the National Institute of Neurological Disorders and Stroke and currently the NVU has definitely come of age [186]. It is the author's current hypothesis that the overarching concept and beauty of the intact NVU is the dynamic interplay or coupling between neural activity and cerebral blood flow and functional hyperemia from which, functional magnetic resonance imaging measures blood oxygenation level dependent (BOLD) images to evaluate human individuals clinically and rodent preclinical models. These images rely on regional differences in cerebral blood flow to delineate regional activity. NVU function may now be considered a multidimensional process involving signals from multiple cells that engage in distinct highly orchestrated signaling pathways and effector systems across the cerebrovascular capillary mesh-like network of NVUs in a 
symphonic-like process. In addition, the NVU with its BBB - blood-brain interface performs as both an endocrine target and an endocrine secretory tissue and therefore, should also be viewed as an endocrine-like organ between the CNS and peripheral tissues, since it is capable of autocrine, paracrine and endocrine-like functions [187]. Furthermore, the NVUs tripartite BBB functions along with its interface functions, which incorporates its signaling to its own supporting cellular network via crosstalk with Pcs, ACs, MGCs, OLs and even neurons provide a vital link to CNS homeostasis.

The multiple weights of multiple cellular injuries in T2DM and their intersects along with the emerging hypotheses of the metabolic hypothesis and antimicrobial protection hypothesis may increase the cellular vulnerability and tip the balance for the brain to develop an increased risk of LOAD (Fig. 31). Further, there may also be novel findings, as in the preclinical $d b / d b$ models, waiting to be gleaned from studying the ultrastructure of short timed post-mortem or biopsies of human brain specimens [188] from T2DM, LOAD and LOAD +T2DM when compared to one another.

Throughout this review multiple efforts have been made to comprehensively utilize supportive ultrastructural TEM images from the cortical grey matter capillary NVUs of layer III and its constitutive cells from the obese, insulin resistant, diabetic $d b / d b$ preclinical mouse model.

For most of the adult life, the brain neurons do not experience exposure to peripheral neurotoxins in health; however, the aged brain and especially those individuals who develop T2DM in mid-life and older age groups experience exposure to peripheral neurotoxins as a result of the NVUs barrier loss of integrity. Importantly, brain neurons are not properly equipped to respond to this increase in exposure to peripherally derived neurotoxins and therefore remain dependent on an intact NVU with barrier integrity for proper homeostasis and function. Therefore, the ultrastructural cellular remodeling changes as shared in this $d b / d b$ diabetic model along with the associated functional changes demonstrate the important role of how structure and function are tightly related to the importance of obesity, aging, intersects and the continuum of progression in regards to the presence of T2DM and the increased risk of LOAD.

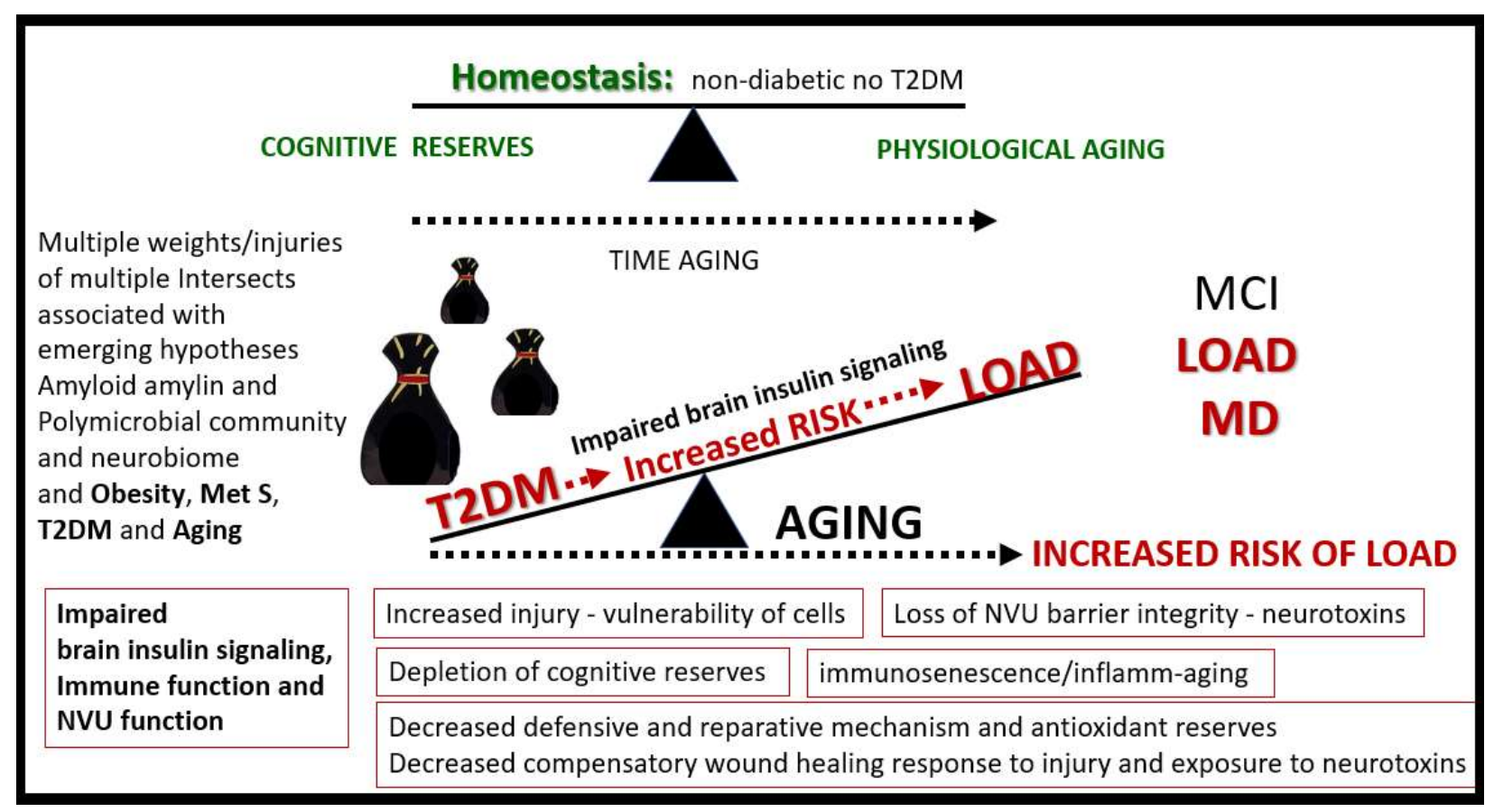

Figure 31. T2DM tips the balance for the development of LOAD. The multiple weights of injuries, metabolic insults, impaired brain insulin signaling, immune function, NVU dysfunction, intersects, ongoing chronic wounding of obesity, MetS, T2DM, aging and the increased burdensome weight of wounding injuries (multifactorial insults and injuries) set in motion the multiple wound healing responses to injury mechanisms with resulting increases in cellular vulnerability along with impaired signaling mechanisms for the development and increased risk of age-related LOAD. LOAD = lateonset Alzheimer's disease; $M C I=$ mild cognitive impairment; $M D=$ mixed dementia; Met $S=$ metabolic syndrome; $N V U=$ neurovascular unit; T2DM = type 2 diabetes mellitus. 


\section{List of Abbreviations}

$\mathrm{A} \alpha=$ Amyloid alpha

$\mathrm{AC}=$ astrocyte

$\mathrm{A} \beta=$ amyloid beta

ACfp $=$ astrocyte foot processes

$\mathrm{AD}=$ Alzheimer's disease

$\mathrm{AFib}=$ amyloid fibrinogen/fibrin

$\mathrm{AGE}=$ advanced glycation end-products

$\mathrm{AMP}=$ antimicrobial peptide(s)

aMt $=$ aberrant mitochondria

$\mathrm{APP}=$ amyloid precursor protein

aMGC $=$ activated microglia cell

$\mathrm{BB}=$ baby boom generation following WWII

Boomer $=$ baby boom generation following WWII

$\mathrm{BBB}=$ blood-brain barrier

$\mathrm{BIR}=$ brain insulin resistance

$\mathrm{BM}=$ basement membrane

$\mathrm{CAA}=$ cerebral amyloid angiopathy

$\mathrm{CBF}=$ cerebral blood flow

$\mathrm{CKC}=\mathrm{C} 57 \mathrm{~B} 6-\mathrm{J}$ control models

$\mathrm{CSF}=$ cerebral spinal fluid

$\mathrm{CVD}=$ cerebro-cardiovascular disease

$\mathrm{DBC}=$ diabetic $d b / d b$ models

$\mathrm{DC}=$ diabetic cognopathy

$\mathrm{DIO}=$ diet induced obesity

$\mathrm{EC}=$ endothelial cell

ecGCx = endothelial cell glycocalyx

eNOS $=$ endothelial nitric oxide synthase

$\mathrm{FFA}=$ free fatty acids

HPA = Hypothalamic-Pituitary-Adrenal (HPA) axis

$\mathrm{H}$ and $\mathrm{E}=$ hyper and excess phenomenon

HSV-1 - herpes simplex virus type 1

$\mathrm{IAPP}=$ islet amyloid polypeptide

IR =insulin resistance

$\mathrm{LOAD}=$ late onset Alzheimer's disease

$\mathrm{MC}=$ metabolic cognopathy

$\mathrm{MD}=$ mixed dementia

MetS $=$ metabolic syndrome

MGC $=$ microglia cell

$\mathrm{MT}=$ microtubule

$\mathrm{NO}=$ nitric oxide

NFT $=$ neurofibrillary tangles 
$\mathrm{NVU}=$ neurovascular unit

$\mathrm{OL}=$ oligodendrocyte

Pc $=$ pericyte

Pcfp $=$ pericyte foot process

$\mathrm{PHF}=$ paired helical fragments

$\mathrm{PIR}=$ peripheral insulin resistance

RAAS = renin-angiotensin-aldosterone system

RAGE $=$ receptor for advanced glycation end-products

$\mathrm{RBC}=$ red blood cell

rMGC $=$ ramified microglia cell

ROS/RNS = reactive oxygen species/ reactive nitrogen species

T2DM = type 2 diabetes mellitus

Tau $=$ microtubule associated protein

$\mathrm{TJ} / \mathrm{AJ}=$ tight junctions/adherens junctions

$\mathrm{VaD}: \mathrm{VAD}=$ vascular dementia

$\mathrm{VCID}=$ vascular contributions of impaired cognition and dementia

VSMC = vascular smooth muscle cell

$\mathrm{WBC}=$ white blood cell

\section{Funding}

This review was supported by an internal grant entitled Excellence in Electron Microscopy to M. R. H. by the Transmission Electron Microscope Core Facility and Office of Research: University of Missouri, Columbia, Missouri. There were no external grants provided to prepare this manuscript.

\section{Acknowledgments}

The author wishes to thank Vincent G. DeMarco for supplying the animal models to study and encouragement in preparing this manuscript; Annayya R. Aroor for obtaining the brain specimens; DeAna G. Grant for preparing the biological tissues and for electron microscope assistance; and Tomi White the director of the University of Missouri Electron Microscopy Core Facility.

\section{Conflicts of Interest}

Author has none to declare.

\section{References}

1. Herculano-Houzel S: The Human Brain in Numbers: A Linearly Scaled-up Primate Brain. Front Hum Neurosci. 2009; 3: 31. doi: 10.3389/neuro.09.031.2009

2. von Bartheld CS, Bahney J, Herculano-Houzel S: The Search for True Numbers of Neurons and Glial Cells in the Human Brain: A Review of 150 Years of Cell Counting. J Comp Neurol. 2016; 524(18): 3865-3895. doi:10.1002/cne.24040

3. Drachman DA: Do we have brain to spare? Neurology. 2005 Jun 28;64(12):2004-2005 doi: https://doi.org/10.1212/01.WNL.0000166914.38327.BB

4. Hayden MR, Grant DG, Aroor AR, Demarco VG: Ultrastructural Remodeling of The Neurovascular Unit in The Female Diabetic db/db Model-Part I: Astrocyte. Neuroglia. 2018;1.220244. doi.org/10.3390/neuroglia1010015

5. Hayden MR, Grant DG, Aroor AR, Demarco VG: Ultrastructural Remodeling of The Neurovascular Unit in The Female Diabetic db/db Model-Part II: Microglia and Mitochondria. Neuroglia 2018, 1, 311326. doi.org/10.3390/neuroglia1020021

6. Hayden MR, Grant DG, Aroor AR, DeMarco VG: Ultrastructural Remodeling of The Neurovascular Unit in The Female Diabetic db/db Model-Part III: Oligodendrocyte and Myelin. Neuroglia 2018, 1, 311326. doi.org/10.3390/neuroglia1020024 
7. Hayden MR, Grant DG, Aroor AR, Demarco VG: Empagliflozin Ameliorates Type 2 Diabetes-Induced Ultrastructural Remodeling of the Neurovascular Unit and Neuroglia in the Female $d b / d b$ Mouse. Brain Sci. 2019;9(3). pii: E57. doi: 10.3390/brainsci9030057

8. Hayden MR, Banks WA, Shah GN, Gu Z, Sowers JR: Cardiorenal metabolic syndrome and diabetic cognopathy. Cardiorenal Med. 2013 Dec;3(4):265-82. doi: 10.1159/000357113.

9. Salameh TS, Shah GN, Price TO, Hayden MR, Banks WA: Blood-Brain Barrier Disruption and Neurovascular Unit Dysfunction in Diabetic Mice: Protection with the Mitochondrial Carbonic Anhydrase Inhibitor Topiramate. J Pharmacol Exp Ther. 2016;359(3):452-459. doi:10.1124/jpet.116.237057

10. Bell RD, Zlokovic BV: Neurovascular mechanisms and blood-brain barrier disorder in Alzheimer's disease. Acta Neuropathol. 2009;118(1):103-13. doi: 10.1007/s00401-009-0522-3

11. Wrighten SA, Piroli GG, Grillo CA, Reagan LP: A look inside the diabetic brain: Contributors to diabetes induced brain aging. Biochim Biophys Acta. 2009; 1792(5): 444-453. doi:10.1016/j.bbadis.2008.10.013.

12. DeVries JH, Desouza C, Bellary S, Unger J, Hansen OKH, Zacho J, Woo V: Achieving glycaemic control without weight gain, hypoglycaemia, or gastrointestinal adverse events in type 2 diabetes in the SUSTAIN clinical trial programme. Diabetes Obes Metab. 2018;20(10):2426-2434. doi: 10.1111/dom.13396.

13. Reasner CA, Defronzo RA. Treatment of type 2 diabetes mellitus: a rational approach based on its pathophysiology. Am Fam Physician. 2001 May 1;63(9):1687-8, 1691-2, 1694.

14. DeFronzo RA, Riccardd C Bonadonna RC, Ferrannini E: Pathogenesis of NIDDM: A Balanced Overview. Diabetes Care 1992; 15(3): 318-368. https://doi.org/10.2337/diacare.15.3.31888.

15. Dagogo-Jack $S^{1}$, Santiago JV: Pathophysiology of type 2 diabetes and modes of action of therapeutic interventions. Arch Intern Med. 1997;157(16):1802-1817.

16. The Diabetes Prevention Program Research Group: Crandall J, Schade D, Ma Y, Fujimoto WY, BarrettConnor E, Fowler S, Dagogo-Jack S, Andres R: The Influence of Age on the Effects of Lifestyle Modification and Metformin in Prevention of Diabetes. J Gerontol A Biol Sci Med Sci. 2006;61(10): 1075-1081.

17. United Nations Department of Economics and Social Affairs Populations Division: Population ageing and sustainable development. June 2017; No2017/1. https://www.un.org/development/desa/publications/world -population-prospects-the-2017-revision.html

18. Tobore TO: On the Etiopathogenesis and Pathophysiology of Alzheimer's Disease: A Comprehensive Theoretical Review. J Alzheimers Dis. 2019 Feb 8. doi: 10.3233/JAD-181052.

19. International Diabetes Foundation. IDF Diabetes Atlas 7th ed. Available from www.diabetesatlas.org. Accessed 1 July 2019.

20. Inzucchi SE, Viscoli CM, Young LH, Furie KL, Gorman M, Lovejoy AM, Dagogo-Jack S, Ismail-Beigi F, Korytkowski MT, Pratley RE, Schwartz GG, Kernan WN; IRIS Trial Investigators: Pioglitazone Prevents Diabetes in Patients With Insulin Resistance and Cerebrovascular Disease. Diabetes Care. 2016 Oct;39(10):1684-92. doi: 10.2337/dc16-0798.

21. Sperling RA, Aisen PS, Beckett LA, Bennett DA, Craft S, Fagan AM, et al: Iwatsubo T, Jack CR Jr, Kaye J, Montine TJ, Park DC, Reiman EM, Rowe CC, Siemers E, Stern Y, Yaffe K, Carrillo MC, Thies B, MorrisonBogorad M, Wagster MV, Phelps CH: Toward defining the preclinical stages of Alzheimer's disease: recommendations from the National Institute on Aging-Alzheimer's Association workgroups on diagnostic guidelines for Alzheimer's disease. Alzheimers Dement. 2011 May; 7(3):280-92. doi: 10.1016/j.jalz.2011.03.003

22. Reaven GM: Banting lecture 1988. Role of insulin resistance in human disease. Diabetes. 1988 Dec;37(12):1595-607.

23. Grundy SM, Cleeman JI, Daniels SR, Donato KA, Eckel RH, Franklin BA, et al. Diagnosis and management of the metabolic syndrome. An American Heart Association/National Heart, Lung, and Blood Institute Scientific Statement. Circulation. 2005;112:2735-2752, https://doi.org/10.1161/CIRCULATIONAHA.105.169 404

24. Ferreira LSS, Fernandes CS, Vieira MNN, De Felice FG: Insulin Resistance in Alzheimer's Disease. Front Neurosci. 2018;12:830. doi: 10.3389/fnins.2018.00830

25. Chatterjee S, Mudher A: Alzheimer's Disease and Type 2 Diabetes: A Critical Assessment of the Shared Pathological Traits. Front Neurosci. 2018;12:383. doi: 10.3389/fnins.2018.00383

26. Pruzin JJ, Nelson PT, Abner EL, Arvanitakis Z: Review: Relationship of type 2 diabetes to human brain pathology. Neuropathol Appl Neurobiol. 2018 Jun;44(4):347-362. doi: 10.1111/nan.12476.

27. Muniyappa R, Sowers JR: Role of insulin resistance in endothelial dysfunction. Rev Endocr Metab Disord. 2013;14(1):5-12. doi: 10.1007/s11154-012-9229-1.

28. Barbagallo M, Dominguez LJ: Type 2 diabetes mellitus and Alzheimer's disease. World J Diabetes. 2014 Dec 15;5(6):889-93. doi: 10.4239/wjd.v5.i6.889 
29. Brownlee M: The pathobiology of diabetic complications: a unifying mechanism. Diabetes. 2005 Jun;54(6):1615-25. https://doi.org/10.2337/diabetes.54.6.1615

30. Strachan MW, Reynolds RM, Frier BM, Mitchell RJ, Price JF: The relationship between type 2 diabetes and dementia. Br Med Bull. 2008;88(1):131-46. doi: 10.1093/bmb/ldn042.

31. Swierczynska MM, Mateska I, Peitzsch M, Bornstein SR, Chavakis T, Eisenhofer G, Lamounier-Zepter $\mathrm{V}$, Eaton S: Changes in morphology and function of adrenal cortex in mice fed a high-fat diet. Int J Obes (Lond). 2015;39(2):321-30. doi: 10.1038/ijo.2014.102

32. Kawarazaki W, Fujita T: The Role of Aldosterone in Obesity-Related Hypertension. Am J Hypertens. 2016 Apr; 29(4): 415-423. doi: 10.1093/ajh/hpw003

33. DeMarco VG, Habibi J, Jia G, Aroor AR, Ramirez-Perez FI, Martinez-Lemus LA, Bender SB, Garro M, Hayden MR, Sun Z, Meininger GA, Manrique C, Whaley-Connell A, Sowers JR: LowDose Mineralocorticoid Receptor Blockade Prevents Western Diet-Induced Arterial Stiffening in Female Mice. Hypertension. 2015 Jul;66(1):99-107. doi: 10.1161/HYPERTENSIONAHA.115.05674

34. Jia G, Habibi J, Aroor AR, Martinez-Lemus LA, DeMarco VG, Ramirez-Perez FI, Sun Z, Hayden MR, Meininger GA, Mueller KB, Jaffe IZ, Sowers JR: Endothelial Mineralocorticoid Receptor Mediates DietInduced Aortic Stiffness in Females. Circ Res. 2016 Mar 18;118(6):935-943. doi: 10.1161/CIRCRESAHA.115.308269

35. Masters CL, Bateman R, Blennow K, Rowe CC, Sperling RA, Cummings JL: Alzheimer's disease. Nat Rev Dis Primers. 2015;1:15056. doi: 10.1038/nrdp.2015.56

36. Jayaraman A, Pike CJ: Alzheimer's disease and type 2 diabetes: multiple mechanisms contribute to interactions. Curr Diab Rep. 2014;14(4):476.

37. Zlokovic BV: Neurovascular mechanisms of Alzheimer's neurodegeneration. Trends Neurosci. 2005;28:202208. doi:10.1016/j.tins.2005.02.001

38. Ott A, Stolk RP, van Harskamp F, Pols HA, Hofman A, Breteler MM: Diabetes mellitus and the risk of dementia: The Rotterdam Study. Neurology. 1999;53:1937-1942. doi: https://doi.org/10.1212/WNL.53.9.1937

39. Arvanitakis Z, Wilson RS, Bienias JL, et al. Diabetes mellitus and risk of Alzheimer disease and decline in cognitive function. Arch Neurol. 2004;61:661-666. doi:10.1001/archneur.61.5.661

40. Luchsinger JA, Tang MX, Shea S, Mayeux R. Hyperinsulinemia and risk of Alzheimer disease. Neurology. 2004;63:1187-1192. doi.org/10.1212/01.WNL.0000140292.04932.87

41. Cheng G, Huang C, Deng H, Wang H. Diabetes as a risk factor for dementia and mild cognitive impairment: a meta-analysis of longitudinal studies. Intern Med J. 2012;42:484-491. doi: 10.1111/j.14455994.2012.02758.x.

42. Whitmer RA, Gunderson EP, Barrett-Connor E, Quesenberry CP. Jr, Yaffe K: (2005). Obesity in middle age and future risk of dementia: a 27 year longitudinal population based study. BMJ. 2005;330: 1360. doi: 10.1136/bmj.38446.466238.E0

43. Nguyen JC, Killcross AS, Jenkins T: Obesity and cognitive decline: role of inflammation and vascular changes. Front Neurosci. 2014 Nov 19;8:375. doi: 10.3389/fnins.2014.00375

44. Gustafson DR, Backman K, Joas E, Waern M, Ostling S, Guo X et al: 37 years of body mass index and dementia: observations from the prospective population study of women in Gothenburg, Sweden. J. Alzheimers. Dis. 2012; 28, 163-171. doi: 10.3233/jad-2011-110917

45. Solfrizzi V, Panza F, Colacicco AM, D’Introno A, Capurso C, Torres F et al: Vascular risk factors, incidence of MCI, and rates of progression to dementia. Neurology. 2004; 63:1882-1891. doi: 10.1212/01.WNL.0000144281.38555.E3

46. Besser LM, Gill DP, Monsell SE, Brenowitz W, Meranus DH, Kukull W et al: (2014). Body mass index, weight change, and clinical progression in mild cognitive impairment and Alzheimer disease. Alzheimer Dis. Assoc. Disord. 2014;28, 36-43. doi: 10.1097/WAD.0000000000000005

47. Strachan MW, Reynolds RM, Marioni RE, Price JF: Cognitive function, dementia and type 2 diabetes mellitus in the elderly. Nat Rev Endocrinol. 2011;7(2):108-114. doi: 10.1038/nrendo.2010.228

48. Mendez MF: Early-onset Alzheimer's Disease: Nonamnestic Subtypes and Type 2 AD. Arch Med Res. 2012 Nov; 43(8): 677-685. doi:10.1016/j.arcmed.2012.11.009

49. Abramowitz WD, Keene CD, Hawes SE, Hubbard RA, Longstreth WT Jr, Woltjer RL, Crane PK, Larson EB, Kukull WA: Alzheimer's disease neuropathologic change, Lewy body disease, and vascular brain injury in clinic- and community-based samples. Neurobiol Aging. 2017 May; 53:83-92. doi: 10.1016/j.neurobiolaging.2017.01.017

50. Rahimi J, Kovacs GG: Prevalence of mixed pathologies in the aging brain. Alzheimers Res. Ther. 2014;6:82. doi: 10.1186/s13195-014-0082-1

51. Schneider JA, Arvanitakis Z, Bang W, Bennett DA: Mixed brain pathologies account for most dementia cases in community-dwelling older persons. Neurology. 2007;69(24):2197-2204. 
52. Snyder HM, Corriveau RA, Craft S, Faber JE, Greenberg SM, Knopman D, Lamb BT, Montine TJ, Nedergaard M, Schaffer CB et al: Vascular contributions to cognitive impairment and dementia including Alzheimer's disease. Alzheimer's Dement. 2015, 11, 710-717. doi: 10.1016/j.jalz.2014.10.008

53. Corriveau RA, Bosetti F, Emr M, Gladman JT, Koenig JI, Moy CS, Pahigiannis K, Waddy SP, Koroshetz $\mathrm{W}$ : The science of vascular contributions to cognitive impairment and dementia (VCID): A framework for advancing research priorities in the cerebrovascular biology of cognitive decline. Cell Mol. Neurobiol. 2016, 36, 281-288. doi: 10.1007/s10571-016-0334-7

54.Murphy MP, Corriveau RA, Wilcock DM: Vascular contributions to cognitive impairment and dementia (VCID). Biochim Biophys Acta. 2016;1862(5):857-559. doi: 10.1016/j.bbadis.2016.02.010.

55. Zlokovic BV. Deane R, Sagare AP, Bell RD, Winkler EA: Low-density lipoprotein receptor-related protein1: a serial clearance homeostatic mechanism controlling Alzheimer'samyloid $\beta$-peptide elimination from the brain. J Neurochem. 2010;115(5):1077-1089. doi: 10.1111/j.1471-4159.2010.07002.x.

56. Sweeney MD, Sagare AP, Zlokovic BV: Cerebrospinal fluid biomarkers of neurovascular dysfunction in mild dementia and Alzheimer's disease. J Cereb Blood Flow Metab. 2015;35(7):1055-1068. doi: 10.1038/jcbfm.2015.76.

57. Sweeney MD, Montagne A, Sagare AP, Nation DA, Schneider LS, Chui HC et al.: The disregarded partner of Alzheimer's disease. Alzheimers Dement. 2019;15(1):158-167. doi: 10.1016/j.jalz.2018.07.222

58. Gudala K, Bansal D, Schifano F, Bhansali A: Diabetes mellitus and risk of dementia: A meta-analysis of prospective observational studies. J Diabetes Investig. 2013;4(6):640-650. doi: 10.1111/jdi.12087

59. Xu WL, Atti AR, Gatz M, Pedersen NL, Johansson B, Fratiglioni L: Midlife overweight and obesity increase late-life dementia risk: A population-based twin study. Neurology. 2011;76(18):1568-74. doi: 10.1212/WNL.0b013e3182190d09.

60. Lizama CO, Zovein AC: Polarizing pathways: Balancing endothelial polarity, permeability, and lumen formation. Exp Cell Res. 2013 May 15;319(9):1247-54. doi: 10.1016/j.yexcr.2013.03.028.

61.. Bors L, Toth K, Toth EZ, Bajza A, Csorba A, Sziegeti K, Mathe D, Perlaki G, Orsi G, Toth GK: Age-dependent changes at the blood-brain barrier. A comparative structural and functional study in young adult and middle-aged rats. Brain Res Bull. 2018;139:269-277. doi: 10.1016/j.brainresbull.2018.03.001

62. Yoon JH, Jeong Y: In vivo imaging for neurovascular disease research. Arch Pharm Res. 2019 Mar;42(3):263273. doi: 10.1007/s12272-019-01128-x

63. Reitsma S, Slaaf DW, Vink H, van Zandvoort MA, oude Egbrink MG: The endothelial glycocalyx: composition, functions, and visualization. Pflugers Arch. 2007;454(3):345-359. doi: 10.1007/s00424-0070212-8

64. Haeren RHL, Rijkers K, Schijns OEMG, Dings J, Hoogland G, van Zandvoort MAMJ, Vink H, van Overbeeke JJ: In vivo assessment of the human cerebral microcirculation and its glycocalyx: A technical report. J Neurosci Methods. 2018 Jun 1;303:114-125. doi: 10.1016/j.jneumeth.2018.03.009

65. Luft JH: Fine structures of capillary and endocapillary layer as revealed by ruthenium red. Fed Proc. 1966;25(6):1773-1783.

66. Dogné S, Flamion B, Caron N: Endothelial Glycocalyx as a Shield Against Diabetic Vascular Complications: Involvement of Hyaluronan and Hyaluronidases. Arterioscler Thromb Vasc Biol. 2018;38(7):1427-1439. doi: 10.1161/ATVBAHA.118.310839

67. Ando Y, Okada H, Takemura G, Suzuki K, Takada C, Tomita H, Zaikokuji R et al: , Hotta Y, Miyazaki N, Yano H, Muraki I, Kuroda A, Fukuda H, Kawasaki Y, Okamoto H, Kawaguchi T, Watanabe T, Doi T, Yoshida T, Ushikoshi H, Yoshida S, Ogura S: Brain-Specific Ultrastructure of Capillary Endothelial Glycocalyx and Its Possible Contribution for Blood Brain Barrier. Sci Rep. 2018;8(1):17523. doi: 10.1038/s41598-018-35976-2.

68. Machin DR, Bloom SL Machin DR, Bloom SI, Campbell RA, Phuong TTT, Gates PE, Lesniewski LA, Rondina MT, Donato AJ: Advanced age results in a diminished endothelial glycocalyx. Am J Physiol Heart Circ Physiol. 2018;315(3):H531-H539. https://doi.org/10.1152/ajpheart.00104.2018

69. Varatharaj A, Galea I: The blood-brain barrier in systemic inflammation. Brain, Behavior, and Immunity. 2017;60:1-12. 60 (2017) 1-12. doi: 10.1016/j.bbi.2016.03.010.

70. Hayden MR, Sowers JR, Tyagi SC: The central role of vascular extracellular matrix and basement membrane remodeling in metabolic syndrome and type 2 diabetes: the matrix preloaded. Cardiovasc Diabetol. 2005; 4: 9. doi: 10.1186/1475-2840-4-9

71. Hurtado-Alvarado G, Cabañas-Morales AM, Gómez-Gónzalez B: Pericytes: brain-immune interface modulators. Front Integr Neurosci. 2014 Jan 10;7:80. doi: 10.3389/fnint.2013.00080. 
72. Hayden MR, Yang Y, Habibi J, Bagree SV, Sowers JR: Pericytopathy: oxidative stress and impaired cellular longevity in the pancreas and skeletal muscle in metabolic syndrome and type 2 diabetes. Oxid Med Cell Longev. 2010;3(5):290-303. doi: 10.4161/oxim.3.5.13653

73. Sweeney MD, Ayyadurai S, Zlokovic BV: Pericytes of the neurovascular unit: key functions and signaling pathways. Nat Neurosci. 2016;19(6):771-783. doi: 10.1038/nn.4288

74. Sweeney MD, Zhao Z, Montagne A, Nelson AR, Zlokovic BV: Blood-Brain Barrier: From Physiology to Disease and Back. Physiol Rev. 2019 Jan 1;99(1):21-78. doi: 10.1152/physrev.00050.2017

75. Hall CN, Reynell C, Gesslein B, Hamilton NB, Mishra A, Sutherland BA, O'Farrell FM, Buchan AM, Lauritzen M, Attwell D: Capillary pericytes regulate cerebral blood flow in health and disease. Nature. 2014 Apr 3;508(7494):55-60. doi: 10.1038/nature13165

76. Hamilton NB, Attwell D, Hall CN: Pericyte-mediated regulation of capillary diameter: a component of neurovascular coupling in health and disease. Front Neuroenergetics. 2010;2. pii: 5. doi: 10.3389/fnene.2010.00005

77. Daneman R, Zhou L, Kebede AA, Barres BA: Pericytes are required for blood-brain barrier integrity during embryogenesis. Nature 2010; 468: 562-566. doi: 10.1038/nature09513

78. Bell RD, Winkler EA, Sagare AP, Singh I, LaRue B, Deane R, Zlokovic BV: Pericytes control key neurovascular functions and neuronal phenotype in the adult brain and during brain aging. Neuron 2010; 68: 409-427. doi: 10.1016/j.neuron.2010.09.043

79. Sagare AP, Bell RD, Zhao Z, Ma Q, Winkler EA, Ramanathan A, Zlokovic BV: Pericyte loss influences Alzheimer-like neurodegeneration in mice. Nat Commun. 2013;4:2932. doi: 10.1038/ncomms3932 53.

80. Hayden MR, Tyagi SC, Kolb L, Sowers JR, Khanna R: Vascular ossification-calcification in metabolic syndrome, type 2 diabetes mellitus, chronic kidney disease, and calciphylaxis-calcific uremic arteriolopathy: the emerging role of sodium thiosulfate. Cardiovasc Diabetol. 2005 Mar 18;4:4 doi: 10.1186/1475-2840-4-4

81. Hayden MR, Yang Y, Habibi J, Bagree SV, Sowers JR: Pericytopathy: oxidative stress and impaired cellular longevity in the pancreas and skeletal muscle in metabolic syndrome and type 2 diabetes. Oxid Med Cell Longev. 2010 Sep-Oct;3(5):290-303. doi: 10.4161/oxim.3.5.13653

82. Hayden MR, Karuparthi PR, Habibi J, Lastra G, Patel K, Wasekar C, Manrique CM, Ozerdem U, Stas S, Sowers JR: Ultrastructure of islet microcirculation, pericytes and the islet exocrine interface in the HIP rat model of diabetes. Exp Biol Med (Maywood). 2008 Sep;233(9):1109-23. doi: 10.3181/0709-RM-251.

83. Hayden MR, Karuparthi PR, Habibi J, Wasekar C, Lastra G, Manrique C, Stas S, Sowers JR: Ultrastructural islet study of early fibrosis in the Ren2 rat model of hypertension. Emerging role of the islet pancreatic pericyte-stellate cell. JOP. 2007 Nov 9;8(6):725-38

84. Kacem K, Lacombe P, Seylaz J, Bonvento G: Structural organization of the perivascular astrocyte endfeet and their relationship with the endothelial glucose transporter: a confocal microscopy study. Glia. 1998; 23(1):1-10.doi: https://doi.org/10.1002/(SICI)1098-1136(199805)23:1<1::AID-GLIA1>3.0.CO;2-B

85.Verkhratsky A, Nedergaard M: Physiology of Astroglia. Physiol Rev. 2018;98(1):239-389. doi: 10.1152/physrev.00042.2016

86. Abbott NJ: Astrocyte-endothelial interactions and blood-brain barrier permeability. 2000; J. Anat. 2002;200(6):629-638. doi: 10.1046/j.1469-7580.2002.00064.x

87. Abbott NJ, Rönnbäck L, Hansson E: Astrocyte-endothelial interactions at the blood-brain barrier. Nat Rev Neurosci. 2006;7(1):41-53. DOI:10.1038/nrn1824

88. McConnell, H.L.; Kersch, C.N.; Woltjer, R.L.; Neuwelt, E.A. The Translational Significance of the Neurovascular Unit. J. Biol. Chem. 2017, 292, 762-770. doi: 10.1074/jbc.R116.760215

89. Petzold GC, Murthy VN: Role of astrocytes in neurovascular coupling. Neuron. 2011; 71(5):782-797. doi: 10.1016/j.neuron.2011.08.009

90. Huang L, Nakamura Y, Lo EH, Hayakawa K: Astrocyte signaling in the neurovascular unit after central nervous system injury. Int J Mol Sci. 2019 Jan 11;20(2). pii: E282. doi: 10.3390/ijms20020282.

91. Mishra A, Reynolds JP, Chen Y, Gourine AV, Rusakov DA, Attwell D: Astrocytes mediate neurovascular signaling to capillary pericytes but not to arterioles. Nat Neurosci. 2016;19(12):1619-1627. doi: 10.1038/nn.4428. Epub 2016 Oct 24. Erratum in: Nat Neurosci. 2017 Jul 26;20(8):1189

92. Hayden MR: Hypothesis: Astrocyte Foot Processes Detachment from the Neurovascular Unit in Female Diabetic Mice May Impair Modulation of Information Processing-Six Degrees of Separation. Brain Sci. 2019;9(4). pii: E83. doi: 10.3390/brainsci9040083

93. Lin B, Koibuchi N, Hasegawa Y, Sueta D, Toyama K, Uekawa K, Ma M, Nakagawa T, Kusaka H, KimMitsuyama S: Glycemic control with empagliflozin, a novel selective SGLT2 inhibitor, ameliorates cardiovascular injury and cognitive dysfunction in obese and type 2 diabetic mice. Cardiovasc Diabetol. 2014 Oct 26;13:148. doi: 10.1186/s12933-014-0148-1 
94. Reemst K, Noctor SC, Lucassen PJ, Hol EM: The indispensable roles of microglia and astrocytes during brain development. Front Hum Neurosci. 2016;10:566. eCollection 2016. doi:10.3389/fnhum.2016.00566

95. Menassa DA, Gomez-Nicola D: Microglial Dynamics During Human Brain Development. Front Immunol. 2018; 9: 1014.doi: 10.3389/fimmu.2018.01014

96. Tambuyzer BR. Ponsaerts P, Nouwen EJ: Microglia: Gatekeepers of the central nervous system immunology. J. Leukoc. Biol. 2009, 85, 352-370. doi: 10.1189/jlb.0608385

97. Pósfai B, Cserép C, Orsolits B, Dénes Á: New Insights into Microglia-Neuron Interactions: A Neuron's Perspective. Neuroscience. 2019;405:103-117. doi: 10.1016/j.neuroscience.2018.04.046

98. Liu Y, Li M, Zhang Z, Ye Y, Zhou J: Role of microglia-neuron interactions in diabetic encephalopathy. Ageing Res. Rev. 2018;42:28-39. doi: 10.1016/j.arr.2017.12.005

99. Crotti A, Ransohoff RM: Microglial Physiology and Pathophysiology: Insights from Genome-wide Transcriptional Profiling.Immunity. 2016;44(3):505-515. doi: 10.1016/j.immuni.2016.02.013

100. Ransohoff RM, Perry VH: Microglial physiology: Unique stimuli, specialized responses. Annu. Rev. Immunol. 2009, 27, 119-145. doi: 10.1146/annurev.immunol.021908.132528

101. Ransohoff RM: A polarizing question: Do M1 and M2 microglia exist? Nat. Neurosci. 2016, 19, 987-99. doi: 10.1038/nn.4338

102. Wang M, Roussos P, McKenzie A, Zhou X, Kajiwara Y, Brennand KJ, De Luca GC, Crary JF, Casaccia P, Buxbaum JD, Ehrlich M, Gandy S, Goate A, Katsel P, Schadt E, Haroutunian V, Zhang B: Integrative network analysis of nineteen brain regions identifies molecular signatures and networks underlying selective regional vulnerability to Alzheimer's disease. Genome Med. 2016;8(1):104. doi: 10.1186/s13073016-0355-3

103. Kettenmann H, Hanisch UK, Noda M, Verkhratsky A: Physiology of microglia. Physiol Rev. 2011;91(2):461-553. doi: 10.1152/physrev.00011.2010.

104. Del Rio-Hortega P. Microglia. In: Cytology and Cellular Pathology of the Nervous System, edited by Penfield W. New York: Hoeber, 1932, p. 482-1924 -534.

105. Michalski JP, Kothary R: Oligodendrocytes in a nutshell. Front. Cell. Neurosci. 2015;9:340. doi: 10.3389/fncel.2015.00340

106. Peters, A.; Palay, S.L.; Webster, H. The Fine Structure of the Nervous System-Neurons and Their Supportive Cells - The Cellular Sheaths of Neurons, 3rd ed.; Oxford University Press: New York, NY, USA, 1991; pp. 212-261. ISBN 0-19-506571-9.

107. Pukos, N.; Yoseph, R.; McTigue, D.M. To Be or Not to Be: Environmental Factors that Drive Myelin Formation during Development and after CNS Trauma. Neuroglia 2018;1(1): 63-90; https://doi.org/10.3390/neuroglia1010007

108. Reijmer, Y.D.; Brundel, M.; de Bresser, J.; Kappelle, L.J.; Leemans, A.; Biessels, G.J. Utrecht Vascular Cognitive Impairment Study Group. Microstructural white matter abnormalities and cognitive functioning in type 2 diabetes: A diffusion tensor imaging study. Diabetes Care 2013;36:137-144. doi: 10.2337/dc12-0493

109. van Agtmaal MJM, Houben AJHM, de Wit V, Henry RMA, Schaper NC, Dagnelie PC, van der Kallen CJ, Koster A, Sep SJ, Kroon AA, Jansen JFA, Hofman PA, Backes WH, Schram MT, Stehouwer CDA: Prediabetes Is Associated With Structural Brain Abnormalities: The Maastricht Study. Diabetes Care. 2018 Dec;41(12):2535-2543. doi: 10.2337/dc18-1132

110. Desai MK, Mastrangelo MA, Ryan DA, Sudol KL, Narrow WC, Bowers WJ: Early oligodendrocyte/myelin pathology in Alzheimer's disease mice constitutes a novel therapeutic target. Am J Pathol. 2010;177(3):142235. doi: 10.2353/ajpath.2010.100087

111. Ramos-Rodriguez JJ, Ortiz O, Jimenez-Palomares M, Kay KR, Berrocoso E, Murillo-Carretero MI, Perdomo G, Spires-Jones T, Cozar-Castellano I, Lechuga-Sancho AM, Garcia-Alloza M: Differential central pathology and cognitive impairment in pre-diabetic and diabetic mice. Psychoneuroendocrinology (2013) 38, 24622475. doi: 10.1016/j.psyneuen.2013.05.010.

112. den Heijer T, Vermeer SE, van Dijk EJ, Prins ND, Koudstaal PJ, Hofman A, Breteler MM: Type 2 diabetes and atrophy of medial temporal lobe structures on brain MRI. Diabetologia. 2003;46(12):1604-1610.

113. Brundel M, van den Heuvel M, de Bresser J, Kappelle LJ, Biessels GJ; Utrecht Diabetic Encephalopathy Study Group: Cerebral cortical thickness in patients with type 2 diabetes. J Neurol Sci. 2010 Dec 15;299(12):126-30. doi: 10.1016/j.jns.2010.08.048.

114. Kutuzov N, Flyvbjerg H, Lauritzen M: Contributions of the glycocalyx, endothelium, and extravascular compartment to the blood-brain barrier. Proc Natl Acad Sci U S A. 2018 Oct 2;115(40):E9429-E9438. doi: 10.1073/pnas.1802155115

115. Frederick RL, Shaw JM: Moving Mitochondria: Establishing Distribution of an Essential Organelle. Traffic. 2007; 8(12): 1668-1675. doi:10.1111/j.1600-0854.2007.00644.x 
116. Cheng Y, Bai F:The Association of Tau With Mitochondrial Dysfunction in Alzheimer's Disease. Front Neurosci. 2018;12:163. doi: 10.3389/fnins.2018.00163

117. Sowers KM, Hayden MR: Calcific uremic arteriolopathy: pathophysiology, reactive oxygen species and therapeutic approaches. Oxid Med Cell Longev. 2010;3(2):109-21. doi: 10.4161/oxim.3.2.11354. KIDNEY

118. Hayden MR, Whaley-Connell A, Sowers JR: Renal redox stress and remodeling in metabolic syndrome, type 2 diabetes mellitus, and diabetic nephropathy: paying homage to the podocyte. Am J Nephrol. 2005 Nov-Dec;25(6):553-69.

119. Yang Y, Hayden MR, Sowers S, Bagree SV, Sowers JR: Retinal redox stress and remodeling in cardiometabolic syndrome and diabetes. Oxid Med Cell Longev. 2010 Nov-Dec;3(6):392-403.

120. Hayden MR, Sowers KM, Pulakat L, Joginpally T, Krueger B, Whaley-Connell A, Sowers JR: Possible Mechanisms of Local Tissue Renin-Angiotensin System Activation in the Cardiorenal Metabolic Syndrome and Type 2 Diabetes Mellitus. Cardiorenal Med. 2011;1(3):193-210.

121. Hayden MR, Sowers JR: Isletopathy in Type 2 diabetes mellitus: implications of islet RAS, islet fibrosis, islet amyloid, remodeling, and oxidative stress. Antioxid Redox Signal. 2007 Jul;9(7):891-910

122. Hayden MR, Sowers JR: Redox imbalance in diabetes. Antioxid Redox Signal. 2007 Jul;9(7):865-7.

123. Whaley-Connell AT, Chowdhury NA, Hayden MR, Stump CS, Habibi J, Wiedmeyer CE, Gallagher PE, Tallant EA, Cooper SA, Link CD, Ferrario C, Sowers JR: Oxidative stress and glomerular filtration barrier injury: role of the renin-angiotensin system in the Ren2 transgenic rat. Am J Physiol Renal Physiol. 2006 Dec;291(6):F1308-14

124 Hayden MR, Whaley-Connell A, Sowers JR: Renal redox stress and remodeling in metabolic syndrome, type 2 diabetes mellitus, and diabetic nephropathy: paying homage to the podocyte. Am J Nephrol. 2005 Nov-Dec;25(6):553-69.

125 Hayden MR, Tyagi SC: Neural redox stress and remodeling in metabolic syndrome, type 2 diabetes mellitus, and diabetic neuropathy. Med Sci Monit. 2004;10(12):RA291-307.

126. Hayden MR, Tyagi SC: Myocardial redox stress and remodeling in metabolic syndrome, type 2 diabetes mellitus, and congestive heart failure. Med Sci Monit. 2003;9(7):SR35-52.

117. PMID: 12883468

127. Hunt MJ, Aru GM, Hayden MR, Moore CK, Hoit BD, Tyagi SC: Induction of oxidative stress and disintegrin metalloproteinase in human heart end-stage failure. Am J Physiol Lung Cell Mol Physiol. 2002 Aug;283(2):L239-45.

128. Hayden MR, Tyagi SC: Islet redox stress: the manifold toxicities of insulin resistance, metabolic syndrome and amylin derived islet amyloid in type 2 diabetes mellitus. JOP. 2002;3(4):86-108.

129. Ritov VB, Menshikova EV, He J, Ferrell RE, Goodpaster BH, Kelley DE: Deficiency of subsarcolemmal mitochondria in obesity and type 2 diabetes. Diabetes. 2005 Jan;54(1):8-14.

130. Patti ME, Corvera S: The role of mitochondria in the pathogenesis of type 2 diabetes. Endocr Rev. 2010 Jun;31(3):364-95. doi: 10.1210/er.2009-0027

131. Kerr JS, Adriaanse BA, Greig NH, Mattson MP, Cader MZ, Bohr VA, Fang EF: Mitophagy and Alzheimer's Disease: Cellular and Molecular Mechanisms. Trends Neurosci. 2017;40(3):151-166. doi: 10.1016/j.tins.2017.01.002.

132. Nunomura A, Perry G, Aliev G, Hirai K, Takeda A, Balraj EK, Jones PK, Ghanbari H, Wataya T, Shimohama S, Chiba S, Atwood CS, Petersen RB, Smith MA: Oxidative damage is the earliest event in Alzheimer disease. J Neuropathol Exp Neurol. 2001;60(8):759-67. PMID:11487050 Correct to here Nunomura A 133.

133. Swerdlow RH, Khan SM: A "mitochondrial cascade hypothesis" for sporadic Alzheimer's disease. Med Hypotheses. 2004; 63(1):8-20. PMID: 15193340

134. Swerdlow RH, Khan SM:The Alzheimer's disease mitochondrial cascade hypothesis: an update. Exp Neurol. 2009;218(2):308-15. doi: 10.1016/j.expneurol.2009.01.011.

135. Swerdlow RH, Burns JM, Khan SM. The Alzheimer's disease mitochondrial cascade hypothesis. J Alzheimers Dis. 2010;20 Suppl 2:S265-79. doi: 10.3233/JAD-2010-100339.

136. Swerdlow RH, Burns JM, Khan SM: The Alzheimer's disease mitochondrial cascade hypothesis: progress and perspectives. Biochim Biophys Acta. 2014 Aug;1842(8):1219-31. doi: 10.1016/j.bbadis.2013.09.010.

137. Selkoe DJ, Hardy J:The amyloid hypothesis of Alzheimer's disease at 25 years.

EMBO Mol Med. 2016;8(6):595-608. doi: 10.15252/emmm.201606210

138. Iqbal K, Liu F, Gong CX, Grundke-Iqbal I: Tau in Alzheimer disease and related tauopathies. Curr Alzheimer Res. 2010;7(8):656-64.

139. Du X, Wang X, Geng M: Alzheimer's disease hypothesis and related therapies. Transl Neurodegener. 2018;7:2. doi: 10.1186/s40035-018-0107-y 
140. Kisler K, Nelson AR, Montagne A, Zlokovic BV: Cerebral blood flow regulation and neurovascular dysfunction in Alzheimer disease. Nat Rev Neurosci. 2017 Jul;18(7):419-434. doi: 10.1038/nrn.2017.48.

141. Kelleher RJ, Soiza R: Evidence of endothelial dysfunction in the development of Alzheimer's disease: Is Alzheimer's a vascular disorder? Am J Cardiovasc Dis. 2013;3(4):197-226. PMID: 24224133

142. Ernst E, Matrai A: Altered red and white blood cell rheology in type II diabetes.

Diabetes. 1986;35(12):1412-1415. https://doi.org/10.2337/diab.35.12.1412

143. Carelli-Alinovi C, Misiti F: Erythrocytes as Potential Link between Diabetes and Alzheimer's Disease. Front Aging Neurosci. 2017; 9: 276. doi: 10.3389/fnagi.2017.00276

144. Cruz Hernández JC, Bracko O, Kersbergen CJ, Muse V, Haft-Javaherian M, Berg M: Neutrophil adhesion in brain capillaries reduces cortical blood flow and impairs memory function in Alzheimer's disease mouse models. Nat Neurosci. 2019;22(3):413-420. doi: 10.1038/s41593-018-0329-4

145. AE, Debbins JP, Malek-Ahmadi M, Chen K, Pipe JG, Maze S, Belden C, Maarouf CL, Thiyyagura P, Mo H, Hunter JM, Kokjohn TA, Walker DG, Kruchowsky JC, Belohlavek M, Sabbagh MN, Beach TG: Cerebral blood flow in Alzheimer's disease. Vasc Health Risk Manag. 2012; 8: 599-611. doi: 10.2147/VHRM.S34874

146. Mistur RMosconi LSanti SD et al. Current challenges for the early detection of Alzheimer's disease: brain imaging and CSF studies. J Clin Neurol 2009;5 (4) 153- 166. doi: 10.3988/jcn.2009.5.4

147. Minoshima S Giordani B Berent S Frey KA Foster NL Kuhl DE Metabolic reduction in the posterior cingulate cortex in very early Alzheimer's disease. Ann Neurol 1997;42 (1) 85-94. doi: 10.1002/ana.410420114

148. Baker LD, Cross DJ, Minoshima S, Belongia D, Watson GS, Craft S: Insulin Resistance and Alzheimer-like Reductions in Regional Cerebral Glucose Metabolism for Cognitively Normal Adults With Prediabetes or Early Type 2 Diabetes. Arch Neurol. 2011;68(1):51-57. doi:10.1001/archneurol.2010.225

149. Perl DP: Neuropathology of Alzheimer's disease. Mt Sinai J Med. 2010;77(1):32-42. doi: 10.1002/msj.20157

150. Braak H, Braak E: Neuropathological stageing of Alzheimer-related changes. Acta Neuropathol. 1991;82(4):239 - 259.

151. Smith EE, Greenberg SM: Beta-amyloid, blood vessels and brain function. Stroke. 2009; 40(7): 2601-2606. doi: 10.1161/STROKEAHA.108.536839

152. Hölscher C: Insulin Signaling Impairment in the Brain as a Risk Factor in Alzheimer's Disease. Front Aging Neurosci. 2019 Apr 24;11:88. doi: 10.3389/fnagi.2019.00088

153. Craft S: The Role of Metabolic Disorders in Alzheimer's Disease and Vascular Dementia: Two Roads Converged? Arch Neurol. 2009 March; 66(3): 300-305. doi:10.1001/archneurol.2009.27

154. Arnold SE, Arvanitakis Z, Macauley-Rambach SL, Koenig AM, Wang HY, Ahima RS, Craft S, Gandy S, Buettner C, Stoeckel LE, Holtzman DM, Nathan DM: Brain insulin resistance in type 2 diabetes and Alzheimer disease: concepts and conundrums. Nat Rev Neurol. 2018 Mar;14(3):168-181. doi: 10.1038/nrneurol.2017.185

155. Mielke JG, Taghibiglou C, Liu L, Zhang Y, Jia Z, Adeli K, Wang YT: A biochemical and functional characterization of diet-induced brain insulin resistance. J Neurochem. 2005; 93(6):1568-1578. doi:10.1111/j.1471-4159.2005.03155.x

156. Wadman M: US government sets out Alzheimer's plan. Nature. 2012;485(7399):426-427. doi: $10.1038 / 485426 a$

157. Avgerinos KI, Kalaitzidis G, Malli A, Kalaitzoglou D, Myserlis PG, Lioutas VA: Intranasal insulin in Alzheimer's dementia or mild cognitive impairment: a systematic review. J Neurol. 2018 Jul;265(7):14971510. doi: 10.1007/s00415-018-8768-0

158. Claxton A, Baker LD, Hanson A, Trittschuh EH, Cholerton B, Morgan A, et al. Long-acting intranasal insulin detemir improves cognition for adults with mild cognitive impairment or earlystage Alzheimer's disease dementia. Journal of Alzheimer's disease: JAD. 2015;44(3):897-906.

159. Craft S, Baker LD, Montine TJ, Minoshima S, Watson GS, Claxton A, et al. Intranasal insulin therapy for Alzheimer disease and amnestic mild cognitive impairment: a pilot clinical trial. Archives of neurology. 2012;69(1):29-38.

160. Craft 2017 Craft S, Claxton A, Baker LD, Hanson AJ, Cholerton B, Trittschuh EH, et al. Effects of Regular and Long-Acting Insulin on Cognition and Alzheimer's Disease Biomarkers: A Pilot Clinical Trial. Journal of Alzheimer's disease: JAD. 2017;57(4):1325-1334.

161. Reger MA, Watson GS, Frey WH, 2nd, Baker LD, Cholerton B, Keeling ML, et al. Effects of intranasal insulin on cognition in memory-impaired older adults: modulation by APOE genotype. Neurobiology of aging. 2006;27(3):451-458.

162. Reger MA, Watson GS, Green PS, Baker LD, Cholerton B, Fishel MA, et al. Intranasal insulin administration dose-dependently modulates verbal memory and plasma amyloid-beta in memory-impaired older adults. Journal of Alzheimer's disease: JAD. 2008;13(3):323-331. 
163. Reger MA, Watson GS, Green PS, Wilkinson CW, Baker LD, Cholerton B, et al. Intranasal insulin improves cognition and modulates beta-amyloid in early AD. Neurology. 2008;70(6):440-448.

164. Liu Y, Liu F, Grundke-Iqbal I, Iqbal K, Gong CX: Deficient brain insulin signalling pathway in Alzheimer's disease and diabetes. J Pathol. 2011 Sep; 225(1): 54-62. doi: 10.1002/path.2912

165. Liu Y, Liu F, Iqbal K, Grundke-Iqbal I, Gong CX: Decreased glucose transporters correlate to abnormal hyperphosphorylation of tau in Alzheimer disease. FEBS Lett. 2008; 23;582(2):359-364. doi: 10.1016/j.febslet.2007.12.035

166. Bharadwaj P, Wijesekara N, Liyanapathirana M, Newsholme P, Ittner L, Fraser P, Verdile G: The Link between Type 2 Diabetes and Neurodegeneration: Roles for Amyloid- $\beta$, Amylin, and Tau Proteins. J Alzheimers Dis. 2017;59(2):421-432. doi: 10.3233/JAD-161192.

167. Hayden MR ${ }^{1}$, Tyagi SC: "A" is for amylin and amyloid in type 2 diabetes mellitus JOP. 2001;2(4):124-39. PMID:11875249

168. Hayden MR, Tyagi SC, Kerklo MM, Nicolls MR. Type 2 diabetes mellitus as a conformational disease. JOP. 2005;6(4):287-302. PMID:16006679

169. Jackson K, Barisone GA, Diaz E, Jin LW, DeCarli C, Despa F: Amylin deposition in the brain: A second amyloid in Alzheimer disease? Ann Neurol. 2013 Oct;74(4):517-26. doi: 10.1002/ana.23956

170. Despa F, Decarli C: Amylin: what might be its role in Alzheimer's disease and how could this affect therapy? Expert Rev Proteomics. 2013;10(5):403-5. doi: 10.1586/14789450.2013.841549

171. Ly H, Despa F: Hyperamylinemia as a risk factor for accelerated cognitive decline in diabetes. Expert Rev Proteomics. 2015;12(6):575-7. doi: 10.1586/14789450.2015.1104251

172. Srodulski S, Sharma S, Bachstetter AB, Brelsfoard JM, Pascual C, Xie XS, Saatman KE, Van Eldik LJ, Despa F: Neuroinflammation and neurologic deficits in diabetes linked to brain accumulation of amylin. Mol Neurodegener. 2014 Aug 22;9:30. doi: 10.1186/1750-1326-9-30.

173. Ly H, Verma N, Wu F, Liu M, Saatman KE, Nelson PT, Slevin JT, Goldstein LB, Biessels GJ, Despa F: Brain microvascular injury and white matter disease provoked by diabetes-associated hyperamylinemia. Ann Neurol. 2017 Aug;82(2):208-222. doi: 10.1002/ana.24992

174. Fawver JN, Ghiwot Y, Koola C, Carrera W, Rodriguez-Rivera J, Hernandez C, Dineley KT, Kong Y, Li J, Jhamandas J, Perry G, Murray IV: Islet amyloid polypeptide (IAPP): A second amyloid in Alzheimer's disease. Curr Alzheimer Res. 2014;11(10):928-940. doi: 10.2174/1567205011666141107124538

175. Grizzanti J, Corrigan R, Casadesus G: Neuroprotective Effects of Amylin Analogues on Alzheimer's Disease Pathogenesis and Cognition. J Alzheimers Dis. 2018;66(1):11-23. doi: 10.3233/JAD-180433

176. Marrero DG, Crean J, Zhang B, Kellmeyer T, Gloster M, Herrmann K, Rubin R, Fineberg N, Kolterman O: Effect of adjunctive pramlintide treatment on treatment satisfaction in patients with type 1 diabetes. Diabetes Care 2007; 30(2): 210-216.https://doi.org/10.2337/dc06-1026

177. Cortes-Canteli M, Zamolodchikov D, Ahn HJ, Strickland S, Norris EH: Fibrinogen and altered hemostasis in Alzheimer's disease. J Alzheimers Dis. 2012;32(3):599-608. doi: 10.3233/JAD-2012-120820.

178. Cortes-Canteli M, Zamolodchikov D, Ahn HJ, Strickland S, Norris EH: Fibrinogen and altered hemostasis in Alzheimer's disease. J Alzheimers Dis. 2012;32(3):599-608. doi: 10.3233/JAD-2012-120820.

179. Gosztyla ML, Brothers HM, Robinson SR: Alzheimer's Amyloid-B is an antimicrobial peptide: A review of the evidence. J Alzheimers Dis. 2018;62(4):1495-1506. doi:10.3233/JAD-171133

180. Marshall BJ, Warren JR: Unidentified curved bacilli in the stomach of patients with gastritis and peptic ulceration. Lancet. 1984;1(8390):1311-5. doi: 10.1016/s0140-6736(84)91816-6

181. Appleby BS, Nacopoulos D, Milano N, Zhong K, Cummings JL: A Review: Treatment of Alzheimer's disease discovered in repurposed agents. Dement Geriatr Cogn Disord. 2013;35(1-2):1-22. doi: $10.1159 / 000345791$

182. Choi Y, Kim HS, Shin KY, Kim EM, Kim M, Kim HS, Park CH, Jeong YH, Yoo J, Lee JP, Chang KA, Kim S, Suh YH: minocycline attenuates neuronal cell death and improves cognitive impairment in Alzheimer's disease models. Neuropsychopharmacology. 2007;32(11):2393-404. doi:10.1038/sj.npp.1301377

183. Bishop GM, Robinson SR; The amyloid hypothesis: let sleeping dogmas lie? Neurobiol Aging. 2002; 23(6):1101-1105. PMID:12470810

184. Moir RD, Lathe R, Tanzi RE: The antimicrobial protection hypothesis of Alzheimer's disease. Alzheimers Dement. 2018 Dec;14(12):1602-1614. doi: 10.1016/j.jalz.2018.06.3040

185. Fulop T, Witkowski JM, Bourgade K, Khalil A, Zerif E, Larbi A, Hirokawa K, Pawelec G, Bocti C, Lacombe G, Dupuis G, Frost EH: Can an Infection Hypothesis Explain the Beta Amyloid Hypothesis of Alzheimer's Disease? Front Aging Neurosci. 2018;10:224. doi: 10.3389/fnagi.2018.00224

186. Iadecola C: The neurovascular unit coming of age: A journey through neurovascular coupling in health and disease. Neuron. 2017;96(1):17-42. doi: 10.1016/j.neuron.2017.07.030. 
187. Banks WA: The blood-brain barrier as an endocrine tissue. Nat Rev Endocrinol. 2019;15(8):444-455. doi: 10.1038/s41574-019-0213-7

188. Claudio L: Ultrastructural features of the blood-brain barrier in biopsy tissue from Alzheimer's disease patients. Acta Neuropathol. 1996;91(1):6-14. PMID: 8773140 